\title{
The Nonexistence of Vortices for Rotating Bose-Einstein Condensates with Attractive Interactions
}

\author{
Yujin Guo, Yong $\mathrm{Luo}^{\dagger}$ and Wen Yang ${ }^{\ddagger}$
}

July 27, 2020

\begin{abstract}
This article is devoted to studying the model of two-dimensional attractive Bose-Einstein condensates in a trap $V(x)$ rotating at the velocity $\Omega$. This model can be described by the complex-valued Gross-Pitaevskii energy functional. It is shown that there exists a critical rotational velocity $0<\Omega^{*}:=\Omega^{*}(V) \leq \infty$, depending on the general trap $V(x)$, such that for any rotational velocity $0 \leq \Omega<\Omega^{*}$, minimizers (i.e., ground states) exist if and only if $a<a^{*}=\|w\|_{2}^{2}$, where $a>0$ denotes the absolute product for the number of particles times the scattering length, and $w>0$ is the unique positive solution of $\Delta w-w+w^{3}=0$ in $\mathbb{R}^{2}$. If $V(x)=|x|^{2}$ and $0<\Omega<\Omega^{*}(=2)$ is fixed, we prove that, up to a constant phase, all minimizers must be real-valued, unique and free of vortices as $a \nearrow a^{*}$, by analyzing the refined limit behavior of minimizers and employing the non-degenerancy of $w$.
\end{abstract}

Key words: Bose-Einstein condensates; rotational velocity; Gross-Pitaevskii functional; mass concentration

\section{Contents}

1 Introduction 2

1.1 Proof strategy of Theorem $1.3 \ldots \ldots \ldots \ldots \ldots \ldots$

$\begin{array}{lll}2 & \text { Existence of Minimizers } & 7\end{array}$

3 Mass Concentration as $a \nearrow a^{*} \quad 8$

$3.1 L^{\infty}$-uniform convergence as $a \nearrow a^{*} \ldots \ldots \ldots \ldots \ldots \ldots$

4 Refined Limiting Profiles 18

5 Nonexistence of Vortices

5.1 Analysis of the linearized operator $\mathcal{N}_{a} \ldots \ldots \ldots \ldots \ldots$. . . . . . . 27

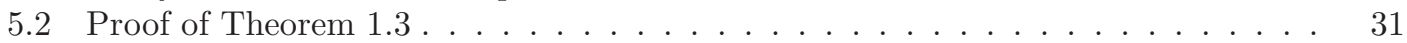

${ }^{*}$ School of Mathematics and Statistics, and Hubei Key Laboratory of Mathematical Sciences, Central China Normal University, Wuhan, 430079, P. R. China. Emails: yjguo@wipm.ac.cn; yguo@ccnu.edu.cn. Y. J. Guo is partially supported by NSFC under Grants No. 11671394 and 11931012.

$\dagger^{\dagger}$ University of Chinese Academy of Sciences, Beijing 100190, P. R. China; Wuhan Institute of Physics and Mathematics, Chinese Academy of Sciences, P.O. Box 71010, Wuhan 430071, P. R. China. Email: luoyong.wipm@outlook.com. Y. Luo is partially supported by the Project funded by China Postdoctoral Science Foundation No. 2019M662680.

$\ddagger$ Wuhan Institute of Physics and Mathematics, Chinese Academy of Sciences, P.O. Box 71010, Wuhan 430071, P. R. China; Innovation Academy for Precision Measurement Science and Technology, Chinese Academy of Sciences, Wuhan 430071, P. R. China. Email: wyang@wipm.ac.cn. W. Yang is partially supported by NSFC under Grant No. 11801550. 


\section{Introduction}

In physical experiments of rotating Bose-Einstein condensates (BECs), a large number of dilute (bosonic) atoms are confined in rotating traps and cooled to the sufficiently low temperatures (cf. $[1,2,9,19,21,23,41])$. The condensation of a large fraction of particles into the same one-particle state can be observed, when the temperature of the system decreases to a critical value. These BECs in rotating traps display various interesting quantum phenomena, such as the appearance of quantized vortices $[1,9,15,23,62,65]$, the center-of-mass rotation $[1,23,47,62]$, the effective lower dimensional behavior in strongly elongated traps [2, 5, 8, 19,21, 23], and so on. Therefore, the properties and their applications of rotating BECs have been a focus of international interest in physics and mathematics since the first physical achievement of rotating BECs in the late 1990s. Especially, the complex structures, including the center-of-mass motions and the quantized vortices of all kinds, of rotating BECs with repulsive interactions have been observed and analyzed extensively over the past two decades, see $[1,2,17-21,23]$ and the references therein.

In contrast to the repulsive case, when the forces between the cold atoms in the condensates are attractive, the system then collapses as the number of the cold atoms increases beyond a critical value, see $[10,11,34,37,54]$ or $[21$, Sec. III.B]. Because of the above distinct mechanism, the system of rotating BECs in the attractive case presents more complicated phenomena and structures, some of which were recently explored by theoretical analysis and numerical simulations, see $[15,21,23,47]$ and the references therein. For example, the collapse cycles and implosion were investigated in three dimensional attractive BECs under rotation. On the other hand, the bright soliton propagation and interactions were however observed in one dimensional attractive BECs under rotation. The main goal of this paper is to investigate mathematically the ground states of two dimensional attractive BECs in rotating traps, which were analyzed recently in $[6,8,30,41]$ and the references therein.

As derived rigorously in [41], the ground states of two-dimensional attractive BECs in rotating traps can be equivalently described (see also $[6,8,51]$ ) by the minimizers of the following complexvalued variational problem:

$$
e_{F}(a):=\inf _{\left\{u \in \mathcal{H},\|u\|_{2}^{2}=1\right\}} F_{a}(u),
$$

where the Gross-Pitaevskii (GP) functional $F_{a}(u)$ is given by

$$
F_{a}(u):=\int_{\mathbb{R}^{2}}\left(|\nabla u|^{2}+V(x)|u|^{2}\right) d x-\frac{a}{2} \int_{\mathbb{R}^{2}}|u|^{4} d x-\Omega \int_{\mathbb{R}^{2}} x^{\perp} \cdot(i u, \nabla u) d x, \quad u \in \mathcal{H},
$$

and the space $\mathcal{H}$ is defined as

$$
\mathcal{H}:=\left\{u \in H^{1}\left(\mathbb{R}^{2}, \mathbb{C}\right): \int_{\mathbb{R}^{2}} V(x)|u|^{2} d x<\infty\right\}
$$

Here $x^{\perp}=\left(-x_{2}, x_{1}\right)$ with $x=\left(x_{1}, x_{2}\right) \in \mathbb{R}^{2}$, and $(i u, \nabla u)=i(u \nabla \bar{u}-\bar{u} \nabla u) / 2$. The parameter $a>0$ in (1.2) characterizes the absolute product of the scattering length $\nu$ of the two-body interaction times the number $N$ of particles in the condensates, and $\Omega \geq 0$ describes the rotational velocity of the trap $V(x)$. Alternatively, one may impose the constraint $\int_{\mathbb{R}^{2}}|u(x)|^{2} d x=N>0$, but the latter case can be easily reduced to the previous one with $a$ being replaced by $a / N$. Therefore, in this paper we shall work with $e_{F}(a)$ instead. Our main interest of investigating $e_{F}(a)$ is on two folds: on one hand, we provide an accurate limit description of minimizers for $e_{F}(a)$ with more general potentials $V(x)$; on the other hand, more importantly we shall prove analytically that up to a constant phase, all minimizers of $e_{F}(a)$ are real valued, unique and free of vortices for some special case.

When there is no rotation for the trap, i.e. $\Omega=0$, the existence, uniqueness, symmetric breaking and the refined limit behavior of real-valued constraint minimizers for $e_{F}(a)$ were studied 
recently in [29-32] and the references therein, see also Theorem 2.1 below for some details. Moreover, the stability and instability of real-valued constraint minimizers for $e_{F}(a)$ with $\Omega=0$ were also studied in $[8,63,64]$ and the references therein. For the above non-rotational case, it turns out that the analysis of $e_{F}(a)$ is connected well with the following nonlinear scalar field equation

$$
-\Delta u+u-u^{3}=0 \text { in } \mathbb{R}^{2} \text {, where } u \in H^{1}\left(\mathbb{R}^{2}, \mathbb{R}\right) .
$$

Recall from $[40,61]$ that, up to translations, (1.4) admits a unique positive solution, which is radially symmetric. For convenience, we denote the unique positive solution of (1.4) by $w=$ $w(|x|)>0$. Note also from [16, Lemma 8.1.2] that $w=w(|x|)>0$ satisfies

$$
\int_{\mathbb{R}^{2}}|\nabla w|^{2} d x=\int_{\mathbb{R}^{2}} w^{2} d x=\frac{1}{2} \int_{\mathbb{R}^{2}} w^{4} d x .
$$

Moreover, it was proved in [61] that the equal sign of the following Gagliardo-Nirenberg inequality

$$
\int_{\mathbb{R}^{2}}|u(x)|^{4} d x \leq \frac{2}{\|w\|_{2}^{2}} \int_{\mathbb{R}^{2}}|\nabla u(x)|^{2} d x \int_{\mathbb{R}^{2}}|u(x)|^{2} d x, \quad u \in H^{1}\left(\mathbb{R}^{2}, \mathbb{R}\right)
$$

is achieved for $u(x)=w(x)$.

More recently, the variational problem $e_{F}(a)$ under rotation $\Omega>0$ was discussed in $[6,8,41]$, where the authors addressed the existence, non-existence and the limit behavior of complexvalued minimizers for $e_{F}(a)$ mainly in the case where $V(x)=|x|^{2}$. Recall from [42] the following diamagnetic inequality: for $\mathcal{A}=\frac{\Omega}{2} x^{\perp}$,

$$
|\nabla u|^{2}-\Omega x^{\perp} \cdot(i u, \nabla u)=|(\nabla-i \mathcal{A}) u|^{2}-\frac{\Omega^{2}}{4}|x|^{2}|u|^{2} \geq|\nabla| u||^{2}-\frac{\Omega^{2}}{4}|x|^{2}|u|^{2}, \quad u \in H^{1}\left(\mathbb{R}^{2}, \mathbb{C}\right) .
$$

By making full use of the inequality (1.7), one can note that the existence and non-existence results of [41] can be extended to the variational problem $e_{F}(a)$ with more general trapping potentials $V(x)$. Concerning on this point, we consider the general trapping potential $0 \leq$ $V(x) \in L_{l o c}^{\infty}\left(\mathbb{R}^{2}\right)$ satisfying

$$
\varliminf_{|x| \rightarrow \infty} \frac{V(x)}{|x|^{2}}>0 .
$$

We also define the critical rotational velocity $\Omega^{*}$ by

$$
\Omega^{*}:=\sup \left\{\Omega>0: \quad V(x)-\frac{\Omega^{2}}{4}|x|^{2} \rightarrow \infty \text { as }|x| \rightarrow \infty\right\} .
$$

As an example, suppose $V(x)=|x|^{s}$ with $s \geq 2$, then we have

$$
\Omega^{*}:=\left\{\begin{array}{lll}
2, & \text { if } & s=2 \\
\infty, & \text { if } & s>2,
\end{array}\right.
$$

which shows that depending on $V(x)$, both $0<\Omega^{*}<\infty$ and $\Omega^{*}=\infty$ can happen. Applying the inequalities (1.6) and (1.7), the arguments of $[30,41]$ give essentially the following existence and nonexistence under the general assumption (1.8):

Theorem 1.1. Let $w$ be the unique positive solution of (1.4). Suppose $V(x) \in L_{\mathrm{loc}}^{\infty}\left(\mathbb{R}^{2}\right)$ satisfies (1.8). Then we have

1. If $0 \leq \Omega<\Omega^{*}$ and $0 \leq a<a^{*}:=\|w\|_{2}^{2}$, then there exists at least one minimizer for $e_{F}(a)$.

2. If $0 \leq \Omega<\Omega^{*}$ and $a \geq a^{*}:=\|w\|_{2}^{2}$, then there is no minimizer for $e_{F}(a)$.

3. If $\Omega>\Omega^{*}$, then for any $a \geq 0$, there is no minimizer for $e_{F}(a)$. 
Moreover, $e_{F}(a)>\inf _{\mathbb{R}^{2}} V_{\Omega}(x)$ for $0 \leq \Omega<\Omega^{*}$ and $a<a^{*}, \lim _{a \rightarrow a^{*}} e_{F}(a)=e_{F}\left(a^{*}\right)=$ $\inf _{\mathbb{R}^{2}} V_{\Omega}(x)$ for $0 \leq \Omega<\Omega^{*}$, and $e_{F}(a)=-\infty$ for $\Omega \geq 0$ and $a>a^{*}$, where

$$
V_{\Omega}(x):=V(x)-\frac{\Omega^{2}}{4}|x|^{2} .
$$

For the reader's convenience, a sketch of the proof for Theorem 1.1 is given in Section 2. From the above conclusion, we have seen that the existence and nonexistence of minimizers for $e_{F}(a)$ are the same as the case without rotation, provided that the rotating velocity $\Omega$ of the trap is smaller than the critical value $\Omega^{*}$. In addition to Theorem 1.1, we note from Remark 2.1 below that for the particular case $\Omega=\Omega^{*}<\infty$, the existence and nonexistence of minimizers for $e_{F}(a)$ seem complicated, which might depend on the exact trapping profile of $V(x)$.

By the variational argument, if $u$ is a minimizer of $e_{F}(a)$, then there exists a Lagrange multiplier $\mu=\mu(a, \Omega) \in \mathbb{R}$ such that $u$ is a complex-valued solution of the following elliptic equation

$$
-\Delta u+V(x) u+i \Omega\left(x^{\perp} \cdot \nabla u\right)=\mu u+a|u|^{2} u \text { in } \mathbb{R}^{2} .
$$

Furthermore, the argument of [30, Theorem 1] implies that if $u_{a}$ is a minimizer of $e_{F}(a)$, then we have

$$
\left\|u_{a}\right\|_{\infty} \rightarrow \infty \text { and } \int_{\mathbb{R}^{2}} V_{\Omega}(x)\left|u_{a}\right|^{2} d x \rightarrow V_{\Omega}\left(x_{0}\right):=\inf _{x \in \mathbb{R}^{2}} V_{\Omega}(x) \text { as } a \nearrow a^{*},
$$

where $V_{\Omega}(x)$ is as in (1.11). This motivates us to investigate the limit behavior of minimizers for $e_{F}(a)$ as $a \nearrow a^{*}$, for which we define

Definition 1.1. The function $h(x) \geq 0$ in $\mathbb{R}^{2}$ is homogeneous of degree $p \in \mathbb{R}^{+}$(with respect to the origin), if there exists some $p>0$ such that

$$
h(t x)=t^{p} h(x) \text { in } \mathbb{R}^{2} \text { for any } t>0 .
$$

Following [28, Remark 3.2], the above definition implies that the homogeneous function $h(x) \in$ $C\left(\mathbb{R}^{2}\right)$ of degree $p>0$ satisfies

$$
0 \leq h(x) \leq C|x|^{p} \text { in } \mathbb{R}^{2} \text {, where } C=\max _{x \in \partial B_{1}(0)} h(x) .
$$

In addition to the assumptions that $V \in L_{\text {loc }}^{\infty}\left(\mathbb{R}^{2}\right)$ satisfies $(1.8)$ and $\Omega^{*}$ is strictly positive, we further assume that $V_{\Omega}(x)=V(x)-\frac{\Omega^{2}}{4}|x|^{2}$ satisfies additionally the following conditions:

$(V) . V_{\Omega}(x) \geq 0, V_{\Omega}(x) \leq C|x|^{q}$ as $|x| \rightarrow \infty$ for some $q \geq 2,\left\{x \in \mathbb{R}^{2}: V_{\Omega}(x)=0\right\}=\{0\}$ and $V_{\Omega}(x)=[1+o(1)] h(x)$ as $x \rightarrow 0$, where $h(x) \in C^{\alpha}\left(\mathbb{R}^{2}\right)$ is homogeneous of degree $p>0$ with $0<\alpha<\min \{p, 1\}$ and $H(y)=\int_{\mathbb{R}^{2}} h(x+y) w^{2}(x) d x$ admits a global minimum point $y_{0} \in \mathbb{R}^{2}$.

Following the above assumption $(V)$, we define $\lambda \in(0, \infty]$ satisfying

$$
\lambda=\left\{\begin{array}{lll}
{\left[\frac{p}{2} \int_{\mathbb{R}^{2}} h\left(x+y_{0}\right) w^{2}(x) d x\right]^{\frac{1}{2+p}},} & \text { if } & 0<p<2 ; \\
{\left[\int_{\mathbb{R}^{2}}\left(h\left(x+y_{0}\right)+\frac{\Omega^{2}}{4}|x|^{2}\right) w^{2}(x) d x\right]^{\frac{1}{4}},} & \text { if } & p=2 ; \\
{\left[\frac{\Omega^{2}}{4} \int_{\mathbb{R}^{2}}|x|^{2} w^{2}(x) d x\right]^{\frac{1}{4}},} & \text { if } & p>2 ;
\end{array}\right.
$$

where the point $y_{0} \in \mathbb{R}^{2}$ is as in the assumption $(V)$. Under the assumptions $(1.8)$ and $(V)$ for some $p>0$, we have the following result concerning the $L^{\infty}$-uniform convergence as $a \nearrow a^{*}$. 
Theorem 1.2. Suppose $V(x) \in L_{\mathrm{loc}}^{\infty}\left(\mathbb{R}^{2}\right)$ satisfies (1.8) and $(V)$ for some $p>0$, and assume $0<\Omega<\Omega^{*}$, where $\Omega^{*}>0$ is defined as in (1.9). If $u_{a}$ is a minimizer of $e_{F}(a)$, then we have

$$
w_{a}(x):=\frac{\left(a^{*}-a\right)^{\frac{1}{2+\gamma}}}{\lambda} u_{a}\left(\frac{\left(a^{*}-a\right)^{\frac{1}{2+\gamma}}}{\lambda} x+x_{a}\right) e^{-i\left(\frac{\Omega}{2 \lambda}\left(a^{*}-a\right)^{\frac{1}{2+\gamma}} x \cdot x_{a}^{\perp}-\theta_{a}\right)} \rightarrow \frac{w(x)}{\sqrt{a^{*}}} \text { as a } \nearrow a^{*}
$$

uniformly in $L^{\infty}\left(\mathbb{R}^{2}, \mathbb{C}\right)$, where $\gamma=\min \{p, 2\}>0$ and $\theta_{a} \in[0,2 \pi)$ is a properly chosen constant. Here $x_{a} \in \mathbb{R}^{2}$ is the unique global maximal point of $\left|u_{a}\right|$ as a $\nearrow a^{*}$.

The proof of Theorem 1.2 relies heavily on the non-degenerancy of $w$ and the delicate energy estimates of $e_{F}(a)$ as well. As mentioned in [41, Remark 2.2], the method of [41] can be used to derive the convergence of (1.17) in an $L^{2}$ sense for the general cases where the trapping potentials attain their minimum at the origin and behave at least quadratically both at zero and infinity, for instance the quartic-quadratic potential $V(x)=|x|^{2}+k|x|^{4}$ for $k \geq 0$. Compared with those obtained in [41], we remark that our Theorem 1.2 holds in the $L^{\infty}$ sense and is applicable to more general cases where trapping potentials attain their minimum at the origin and behave at least quadratically only at infinity, for instance the general potential $V(x)=|x|^{2}+k|x|^{q}$ for $k \geq 0$ and $q>0$.

Finally, we consider a more refined limit behavior of minimizers for $e_{F}(a)$ as $a \nearrow a^{*}$, in the case where $V(x)=|x|^{2}$ and $0<\Omega<\Omega^{*}$ is fixed. In this case, recall from (1.10) that $\Omega^{*}=2$. Based on the $L^{\infty}$-uniform convergence of Theorem 1.2, we shall show the following nonexistence of vortices for $e_{F}(a)$ confined in the harmonic trap.

Theorem 1.3. Suppose $V(x)=|x|^{2}$ and $0<\Omega<\Omega^{*}=2$. Then, up to a constant phase, all minimizers of $e_{F}(a)$ are real-valued, unique and free of vortices as a is sufficiently close to $a^{*}$ from below.

As the main result of the current paper, Theorem 1.3 can be generalized to the case $V(x)=$ $|x-A|^{2}$ for any point $A=\left(a_{1}, a_{2}\right) \in \mathbb{R}^{2}$. This follows directly by the transformation $v(x)=$ $u\left(x+\frac{4 A}{4-\Omega^{2}}\right) e^{-i \frac{2 \Omega}{4-\Omega^{2}} x \cdot A^{\perp}}$, where $A^{\perp}=\left(-a_{2}, a_{1}\right)$. Theorem 1.3 and [29, Theorem 1.4] yield immediately that up to a constant phase $\theta_{a} \in[0,2 \pi)$, the unique minimizer $u_{a}$ of $e_{F}(a)$ satisfies

$$
\begin{aligned}
u_{a}(x)=e^{-i \theta_{a}} u_{a}^{0}(x) \approx & \frac{\lambda}{\|w\|_{2}} e^{-i \theta_{a}}\left\{\frac{1}{\left(a^{*}-a\right)^{\frac{1}{4}}} w\left(\frac{\lambda|x|}{\left(a^{*}-a\right)^{\frac{1}{4}}}\right)+\left(a^{*}-a\right)^{\frac{3}{4}} \psi\left(\frac{\lambda|x|}{\left(a^{*}-a\right)^{\frac{1}{4}}}\right)\right. \\
& \left.+\left(a^{*}-a\right)^{\frac{7}{4}} \phi_{0}\left(\frac{\lambda|x|}{\left(a^{*}-a\right)^{\frac{1}{4}}}\right)+o\left(\left(a^{*}-a\right)^{\frac{7}{4}}\right)\right\} \text { as } a \nearrow a^{*},
\end{aligned}
$$

where $u_{a}^{0}(x)>0$ is the unique real minimizer of $e_{F}(a)$ at $\Omega=0$ as $a \nearrow a^{*}$, and $\lambda>0$ is defined by (1.16) with $p=2$. Here the real-valued radially symmetric functions $\psi, \phi_{0} \in C^{2}\left(\mathbb{R}^{2}\right) \cap L^{\infty}\left(\mathbb{R}^{2}\right)$ are unique. Therefore, the expansion (1.18) gives a positive answer for the question raised soon after [41, Theorem 2.1].

\subsection{Proof strategy of Theorem 1.3}

The purpose of this subsection is to explain the general strategy of proving Theorem 1.3, which can be summarized as the method of inductive symmetry. Roughly speaking, our basic idea is to derive the radial symmetry of minimizers as $a \nearrow a^{*}$ by analyzing the refined limit behavior of minimizers and employing the non-degenerancy of $w$.

To prove Theorem 1.3, the first step is the $L^{\infty}$-uniform convergence already established in Theorem 1.2. Under the assumptions that $V=|x|^{2}$ and $0<\Omega<\Omega^{*}=2$, let $u_{a}$ be a minimizer of $e_{F}(a)$. Stimulated by (1.17), we consider

$$
\begin{aligned}
w_{a}(x) & :=\varepsilon_{a} u_{a}\left(\varepsilon_{a} x+x_{a}\right) e^{-i \Omega\left(\frac{\varepsilon_{a}}{2} x \cdot x_{a}^{\perp}-\theta_{a}\right)} \\
& :=R_{a}(x)+i I_{a}(x), \quad \varepsilon_{a}:=\left(\int_{\mathbb{R}^{2}}\left|\nabla u_{a}\right|^{2} d x\right)^{-\frac{1}{2}}>0,
\end{aligned}
$$


where $\theta_{a} \in[0,2 \pi)$ is chosen properly as in (1.17), and $x_{a} \in \mathbb{R}^{2}$ is the global maximal point of $\left|u_{a}\right|$. Here $R_{a}$ and $I_{a}$ are real-valued functions in $H^{1}\left(\mathbb{R}^{2}\right)$. The proof of Theorem 1.2 then yields the following $L^{\infty}$-uniform convergence

$$
R_{a} \rightarrow \frac{w}{\sqrt{a^{*}}} \text { and } I_{a} \rightarrow 0 \text { uniformly in } \mathbb{R}^{2} \text { as } a \nearrow a^{*},
$$

where the non-degenerancy of $w$ is used.

In the second step, we shall establish Proposition 4.1 on the first estimates of the difference $R_{a}-\frac{w}{\sqrt{a^{*}}}$ and $I_{a}$ as $a \nearrow a^{*}$, by following (1.20) and analyzing the following system:

$$
\left\{\begin{aligned}
\mathcal{L}_{a} R_{a} & =\varepsilon_{a}^{2} \Omega\left(x^{\perp} \cdot \nabla I_{a}\right) & & \text { in } \mathbb{R}^{2}, \\
\mathcal{L}_{a} I_{a} & =-\varepsilon_{a}^{2} \Omega\left(x^{\perp} \cdot \nabla R_{a}\right) & & \text { in } \mathbb{R}^{2},
\end{aligned}\right.
$$

where

$$
\mathcal{L}_{a}:=-\Delta+\left(\frac{\varepsilon_{a}^{4} \Omega^{2}}{4}|x|^{2}+\varepsilon_{a}^{2} V_{\Omega}\left(\varepsilon_{a} x+x_{a}\right)-\varepsilon_{a}^{2} \mu_{a}-a\left|w_{a}\right|^{2}\right) .
$$

Here, the system (1.21) is obtained from (1.12) and (1.19).

The third step is the first estimates of $R_{a}-v_{a}$ and $I_{a}$ as $a \nearrow a^{*}$, where $v_{a}>0$ is the rescaled positive minimizer of $e_{F}(a)$ at zero rotation $\Omega=0$. Note from [29] that $v_{a}>0$ is radially symmetric and unique as $a \nearrow a^{*}$. We should emphasize that we are unable to derive the further refined estimates $R_{a}$ and $I_{a}$ as $a \nearrow a^{*}$ by further analyzing the difference $R_{a}-\frac{w}{\sqrt{a^{*}}}$, which is mainly due to the non-symmetry of $\mathcal{L}_{a}$. The main results of this step are given in Proposition 5.1 by applying Proposition 4.1 and some refined estimates of [29].

Associated to each $v_{a}$, there is a linearized operator $\mathcal{N}_{a}: D\left(\mathcal{N}_{a}\right) \subset L^{2}\left(\mathbb{R}^{2}\right) \longmapsto L^{2}\left(\mathbb{R}^{2}\right)$ defined as

$$
\mathcal{N}_{a}:=-\Delta+\left(\varepsilon_{a}^{4}|x|^{2}-\varepsilon_{a}^{2} \beta_{a}-3 a v_{a}^{2}\right),
$$

where $\varepsilon_{a}>0$ is defined in (1.19), and the domain $D\left(\mathcal{N}_{a}\right)$ is defined by

$$
D\left(\mathcal{N}_{a}\right)=H^{2}\left(\mathbb{R}^{2}\right) \cap\left\{u \in L^{2}\left(\mathbb{R}^{2}\right): \int_{\mathbb{R}^{2}}|x|^{4} u^{2} d x<\infty\right\} .
$$

The fourth step is to study the operator $\mathcal{N}_{a}$ and establish the inductive estimates of Lemma 5.2.

Following the estimates in previous steps, the last step is the complete proof of Theorem 1.3 by an induction process. Heuristically speaking, we shall show that once we have a good control on the non-radial part of $R_{a}-v_{a}$, then a better control on the non-radial part of $R_{a}-v_{a}$ could be obtained. As a consequence, we shall finally prove in Subsection 5.2 that the non-radial part of $R_{a}-v_{a}$ is arbitrarily small and

$$
\text { both } I_{a}(x) \equiv 0 \text { and } x_{a} \equiv 0 \text { as } a \nearrow a^{*} \text {. }
$$

By the uniqueness of $v_{a}>0$ as $a \nearrow a^{*}$ (cf. [29, Theorem 1.3]), we are able to conclude from above and (1.19) that up to a constant phase, all minimizers of $e_{F}(a)$ must be real-valued, unique and free of vortices as $a \nearrow a^{*}$, and thereby Theorem 1.3 is proved. For the details of the proof of Theorem 1.3, we refer the reader to Section 5.

It is worthy of mentioning that the above "inductive symmetry" argument can be applied for any radially symmetric potential like $|x|^{2}+t|x|^{p}$ with positive $t$ and $p \in(1,+\infty)$. Indeed, it can be generalized to the general radially symmetric function which admits polynomial growth at most, provided that the following two points are ensured: at first, Theorem 5.3 still holds for the given potential; secondly, we have to change the assumptions of Lemma 5.4 and Lemma A.1 a little bit by requiring that $\left|T_{m, a}\right|$ and $\left|f_{i}\right|$ decay slightly faster than $\left|\nabla T_{m, a}\right|$ and $\left|\nabla f_{i}\right|$, respectively, where $i=1,2$.

Before we end the introduction, it deserves to remark that the nonexistence of vortices for rotating BECs with repulsive interactions were proved earlier in $[3,4,17-20,35,36,53]$ by different arguments, including jacobian estimates, vortex ball constructions, and so on. We also refer 
the celebrated monograph [56] to various kinds of techniques involved in the investigation of vortex structures for defocusing nonlinear problems. As far as we know, it seems that the above mentioned methods of studying vortex structures concentrate more on the analysis of the energy, which is not enough for our focusing nonlinear case. Indeed, besides the refined analysis of the energy, the proof of our Theorem 1.3 relies more on the refined limit analysis of the governing system (1.21), for which one needs to make full use of the non-degenerancy of $w$.

This paper is organized as follows: in Section 2 we shall sketch briefly the proof of Theorem 1.1 under more general potentials satisfying (1.8). The proof of Theorem 1.2 is given in Section 3 by employing energy methods and blow-up analysis. Section 4 is devoted to the proof of Proposition 4.1 on the refined limit behavior of minimizers for $e_{F}(a)$ as $a \nearrow a^{*}$. In Section 5 , we first establish Proposition 5.1, and the linearized operator $\mathcal{N}_{a}$ is then analyzed in Subsection 5.1. We shall complete the proof of Theorem 1.3 in Subsection 5.2 by the so-called method of inductive symmetry described above. In Appendix A, we shall prove two lemmas which are used in the proof of Theorem 1.3.

\section{Existence of Minimizers}

In this section, we shall address the proof of Theorem 1.1 on the existence and nonexistence of minimizers for $e_{F}(a)$. Since the proof of Theorem 1.1 is overall similar to [30, Theorem 1], we only sketch out the main differences.

Letting $w>0$ be the unique positive radial solution of (1.4), note that $w$ strictly decreases in $|x|$ (cf. [24, Proposition 4.1]), and admits the following exponential decay

$$
w(x),|\nabla w(x)|=O\left(|x|^{-\frac{1}{2}} e^{-|x|}\right) \quad \text { as }|x| \rightarrow \infty,
$$

which are often used throughout the paper. We next recall some results and notations from [30] on the minimizers of $e_{F}(a)$ in the special case where $\Omega=0$. Equivalently, we consider the following Gross-Pitaevskii energy functional

$$
E_{a}(u):=\int_{\mathbb{R}^{2}}\left(|\nabla u|^{2}+V(x)|u|^{2}\right) d x-\frac{a}{2} \int_{\mathbb{R}^{2}}|u|^{4} d x, \quad u \in \mathcal{H}
$$

under the unit mass constraint $\int_{\mathbb{R}^{2}}|u|^{2} d x=1$, i.e.,

$$
e(a):=\inf _{\left\{u \in \mathcal{H} \text { and } \int_{\mathbb{R}^{2}}|u|^{2} d x=1\right\}} E_{a}(u),
$$

where $\mathcal{H}$ is defined as in (1.3). One can note that $E_{a}(|u|) \leq E_{a}(u)$ holds for any $u \in \mathcal{H}$. This property and [30, Theorem 1] yield immediately the following existence and nonexistence:

Theorem 2.1. Let $w$ be the unique positive radial solution of (1.4). Suppose $V \in L_{\text {loc }}^{\infty}\left(\mathbb{R}^{2}\right)$ satisfies $\lim _{|x| \rightarrow \infty} V(x)=\infty$. Then we have

1. If $0 \leq a<a^{*}:=\|w\|_{2}^{2}$, then there exists at least one minimizer for (2.3).

2. If $a \geq a^{*}:=\|w\|_{2}^{2}$, then there is no minimizer for (2.3).

To prove Theorem 1.1 for the rotational case where $\Omega>0$, one needs to use the following diamagnetic inequality (cf. [42]): for $\mathcal{A}=\frac{\Omega}{2} x^{\perp}$,

$$
|\nabla u|^{2}-\Omega x^{\perp} \cdot(i u, \nabla u)=|(\nabla-i \mathcal{A}) u|^{2}-\frac{\Omega^{2}}{4}|x|^{2}|u|^{2} \geq\left.|\nabla| u\right|^{2}-\frac{\Omega^{2}}{4}|x|^{2}|u|^{2}, \quad u \in H^{1}\left(\mathbb{R}^{2}, \mathbb{C}\right) .
$$

Define the critical rotational velocity $\Omega^{*}$ as in (1.9), such that if $V(x)$ satisfies (1.8), then $\Omega^{*}$ exists and satisfies $0<\Omega^{*} \leq \infty$. Similar to those in [63] and references therein, one can establish that for $V \in L_{\text {loc }}^{\infty}\left(\mathbb{R}^{2}\right)$ satisfying $\lim _{|x| \rightarrow \infty} V(x)=\infty$, if $2 \leq q<\infty$, then the embedding $\mathcal{H} \hookrightarrow L^{q}\left(\mathbb{R}^{2}, \mathbb{C}\right)$ is compact. Applying the Gagliardo-Nirenberg inequality (1.6) and the diamagnetic inequality (2.4), similar to [30, Theorem 1] it is standard to use the above compactness property to derive the following existence. 
Theorem 2.2. Let $w=w(|x|)$ be the unique positive radial solution of (1.4). Suppose $V(x) \in$ $L_{\text {loc }}^{\infty}\left(\mathbb{R}^{2}\right)$ satisfies (1.8) such that $\Omega^{*}>0$ exists, where $\Omega^{*}$ is defined as in (1.9). Then for any $0<\Omega<\Omega^{*}$ and $0 \leq a<a^{*}:=\|w\|_{2}^{2}$, there exists at least one minimizer for $e_{F}(a)$.

We now make the following observation: by applying (1.6), the second inequality of (2.4) yields that

$$
F_{a}(u) \geq\left(1-\frac{a}{a^{*}}\right) \int_{\mathbb{R}^{2}}|\nabla| u||^{2} d x+\int_{\mathbb{R}^{2}} V_{\Omega}(x)|u|^{2} d x, \quad u \in \mathcal{H},
$$

where $V_{\Omega}(x)=V(x)-\frac{\Omega^{2}}{4}|x|^{2}$. Choose a non-negative function $\varphi \in C_{0}^{\infty}\left(\mathbb{R}^{2}\right)$ such that $\varphi(x)=1$ for $|x| \leq 1$. For any large $\tau>0$, we then consider the test function

$$
w_{\tau}(x)=A_{\tau} \frac{\tau}{\|w\|_{2}} \varphi\left(x-x_{0}\right) w\left(\tau\left(x-x_{0}\right)\right) e^{i \Omega S(x)},
$$

where $x_{0} \in \mathbb{R}^{2}$ is a suitable point, $S(x)=\frac{1}{2} x \cdot x_{0}^{\perp}$, and $A_{\tau}>0$ is chosen so that $\int_{\mathbb{R}^{2}}\left|w_{\tau}(x)\right|^{2} d x=1$. Employing above estimates and the test function (2.6), the following nonexistence result can be established in the similar way of proving [30, Theorem 1].

Theorem 2.3. Let $w=w(|x|)$ be the unique positive radial solution of (1.4). Suppose $V(x) \in$ $L_{\text {loc }}^{\infty}\left(\mathbb{R}^{2}\right)$ satisfies (1.8) such that $\Omega^{*}>0$ exists, where $\Omega^{*}$ is defined as in (1.9). Then there is no minimizer for $e_{F}(a)$, provided that either

1. $0<\Omega<\Omega^{*}$ and $a \geq a^{*}:=\|w\|_{2}^{2}$, or

2. $\Omega>\Omega^{*}$ and $a \geq 0$.

Moreover, $e_{F}(a)>\inf _{\mathbb{R}^{2}} V_{\Omega}(x)$ for $0 \leq \Omega<\Omega^{*}$ and $a<a^{*}, \lim _{a \nearrow a^{*}} e_{F}(a)=e_{F}\left(a^{*}\right)=$ $\inf _{\mathbb{R}^{2}} V_{\Omega}(x)$ for $0 \leq \Omega<\Omega^{*}$, and $e(a)=-\infty$ for $\Omega \geq 0$ and $a>a^{*}$.

Combining with Theorems 2.1 and 2.2, whose proofs are omitted for simplicity, Theorem 2.3 completes the proof of Theorem 1.1.

Remark 2.1. As the complement of Theorem 1.1, we guess that the existence and nonexistence of minimizers in the case where $\Omega=\Omega^{*}<\infty$ depend on $a$ and $V(x)$. Specifically, consider the trapping profile $V(x)=|x|^{2}$ and $\Omega=\Omega^{*}(=2)$. When $a=0$, by the following inequality (see [22, Remark 2.5])

$$
\int_{\mathbb{R}^{2}}\left|\nabla u-i x^{\perp} u\right|^{2} d x \geq 2 \int_{\mathbb{R}^{2}}|u|^{2} d x
$$

we have $e_{F}(0) \geq 2$, and one can further check that $e_{F}(0) \leq 2$ by using $u=\frac{1}{\sqrt{\pi}} e^{-\frac{|x|^{2}}{2}}$ as a test function. Thus, $e_{F}(0)$ admits a minimizer $u=\frac{1}{\sqrt{\pi}} e^{-\frac{|x|^{2}}{2}}$ in this case. While if $a=a^{*}$, we have $F_{a^{*}}(u) \geq 0$, and we can further verify that $e_{F}\left(a^{*}\right)=0$ by taking $u_{\tau}=\frac{1}{\sqrt{a^{*}}} \tau w(\tau x)$ as a test function and letting $\tau \rightarrow \infty$. Then, it is not difficult to check that there is no minimizer in the case where $a=a^{*}$.

\section{Mass Concentration as $a \nearrow a^{*}$}

By employing energy methods and blow-up analysis, the main purpose of this section is to establish Theorem 1.2 on the $L^{\infty}$-uniform convergence of minimizers for $e_{F}(a)$ as $a \nearrow a^{*}$. Recall from Theorem 1.1 that $e_{F}(a)$ admits minimizers and $e_{F}(a) \rightarrow \inf _{\mathbb{R}^{2}} V_{\Omega}(x)$ as $a \nearrow a^{*}$, where $V_{\Omega}(x)$ satisfies the assumption $(V)$ for any $p>0$, and $e_{F}(a)$ is the GP energy defined in (1.1).

To prove Theorem 1.2, we first address the following energy estimates of $e_{F}(a)$ as $a \nearrow a^{*}$. 
Lemma 3.1. Suppose that $V(x) \in L_{\mathrm{loc}}^{\infty}\left(\mathbb{R}^{2}\right)$ satisfies (1.8) and $(V)$ for some $p>0$, and assume $0<\Omega<\Omega^{*}$, where $\Omega^{*}>0$ is defined in (1.9). Then there exist two positive constants $m$ and $M$, independent of $0<a<a^{*}$, such that

$$
0<m\left(a^{*}-a\right)^{\frac{p}{p+2}} \leq e_{F}(a) \leq M\left(a^{*}-a\right)^{\frac{\gamma}{\gamma+2}} \text { as a } \nearrow a^{*},
$$

where $\gamma=\min \{p, 2\}>0$.

Proof. By (2.5), we first note that a similar argument of [30, Lemma 3] gives the lower bound of (3.1).

To prove the upper bound of (3.1), similar to Theorem 2.3, we use a test function $w_{\tau}$ of the form (2.6) with $S(x) \equiv 0$, where $A_{\tau}>0$ satisfies

$$
\frac{1}{A_{\tau}^{2}}=\frac{1}{\|w\|_{2}^{2}} \int_{\mathbb{R}^{2}} w(x)^{2} \varphi(x / \tau)^{2} d x=1+O\left(\tau^{-\infty}\right) \quad \text { as } \tau \rightarrow \infty,
$$

due to the exponential decay of $w$ in Eq. (2.1), where and below we always use $f(t)=O\left(t^{-\infty}\right)$ to denote a function $f$ satisfying $\lim _{t \rightarrow \infty}|f(t)| t^{s}=0$ for all $s>0$. Under the assumption $(V)$ for some $p>0$, choose $\mathcal{R}>0$ small enough so that $V_{\Omega}(x) \leq C_{0}|x|^{p}$ for $|x| \leq \mathcal{R}$, where $V_{\Omega}(x)$ is defined as in (1.11). In view of this fact, we have

$$
\int_{\mathbb{R}^{2}} V_{\Omega}(x)\left|w_{\tau}(x)\right|^{2} d x \leq \frac{C_{0} A_{\tau}^{2}}{\tau^{p}} \int_{\mathbb{R}^{2}}|x|^{p} w^{2}(x) d x \quad \text { as } \tau \rightarrow \infty .
$$

We then obtain from (2.6) that

$$
\begin{aligned}
C_{\tau}\left(w_{\tau}\right): & =\int_{\mathbb{R}^{2}}\left(\left|\nabla w_{\tau}\right|^{2}-\Omega x^{\perp} \cdot\left(i w_{\tau}, \nabla w_{\tau}\right)+\frac{\Omega^{2}}{4}|x|^{2}\left|w_{\tau}\right|^{2}\right) d x-\frac{a}{2} \int_{\mathbb{R}^{2}}\left|w_{\tau}\right|^{4} d x \\
& =\frac{\tau^{2}}{2\|w\|_{2}^{2}}\left(1-\frac{a}{a^{*}}\right) \int_{\mathbb{R}^{2}} w^{4}(x) d x+\frac{\Omega^{2}}{4\|w\|_{2}^{2} \tau^{2}} \int_{\mathbb{R}^{2}}|x|^{2} w^{2}(x) d x+O\left(\tau^{-\infty}\right)
\end{aligned}
$$

as $\tau \rightarrow \infty$. Thus, we conclude from above that

$$
\begin{aligned}
e_{F}(a) \leq & \frac{\tau^{2}}{2\left(a^{*}\right)^{2}}\left(a^{*}-a\right) \int_{\mathbb{R}^{2}} w^{4}(x) d x+\frac{C_{0}}{\tau^{p}} \int_{\mathbb{R}^{2}}|x|^{p} w^{2}(x) d x \\
& +\frac{\Omega^{2}}{4\|w\|_{2}^{2} \tau^{2}} \int_{\mathbb{R}^{2}}|x|^{2} w^{2}(x) d x+O\left(\tau^{-\infty}\right) \text { as } \tau \rightarrow \infty .
\end{aligned}
$$

Optimizing the right hand side of (3.3) by taking

$$
\tau=\left(a^{*}-a\right)^{-\frac{1}{\gamma+2}}, \quad \text { where } \gamma:=\min \{p, 2\}>0,
$$

we arrive at the desired upper bound of (3.1), and the proof is therefore complete.

For a given $0<\Omega<\Omega^{*}$, if $u_{a}$ is a minimizer of $e_{F}(a)$ for $0<a<a^{*}$, then $u_{a}$ is a solution of the following elliptic equation

$$
-\Delta u_{a}+V(x) u_{a}+i \Omega\left(x^{\perp} \cdot \nabla u_{a}\right)=\mu_{a} u_{a}+a\left|u_{a}\right|^{2} u_{a} \quad \text { in } \mathbb{R}^{2},
$$

where $\mu_{a} \in \mathbb{R}$ is a Lagrange multiplier. One can check that $\mu_{a}$ satisfies

$$
\mu_{a}=e_{F}(a)-\frac{a}{2} \int_{\mathbb{R}^{2}}\left|u_{a}\right|^{4} d x, 0<a<a^{*} .
$$

The following lemma gives the refined estimates of $u_{a}$ and $\mu_{a}$ as $a \nearrow a^{*}$.

Lemma 3.2. Set

$$
\varepsilon_{a}:=\left(\int_{\mathbb{R}^{2}}\left|\nabla u_{a}\right|^{2} d x\right)^{-\frac{1}{2}}>0,
$$

where $u_{a}$ is a minimizer of $e_{F}(a)$. Suppose that the assumption of Theorem 1.2 holds. Then we have 
1. $\varepsilon_{a}>0$ satisfies $\varepsilon_{a} \rightarrow 0$ and $\mu_{a} \varepsilon_{a}^{2} \rightarrow-1$ as a $\nearrow a^{*}$.

2. Define the normalized function as follows:

$$
w_{a}(x):=\varepsilon_{a} u_{a}\left(\varepsilon_{a} x+x_{a}\right) e^{-i\left(\theta_{a}+\frac{\varepsilon_{a} \Omega}{2} x \cdot x_{a}^{\perp}\right)},
$$

where $x_{a}$ is a global maximal point of $\left|u_{a}\right|$ and $\theta_{a} \in[0,2 \pi)$ is a proper constant. Then there exists a constant $\eta>0$, independent of $0<a<a^{*}$, such that

$$
\int_{B_{2}(0)}\left|w_{a}(x)\right|^{2} d x \geq \eta>0 \text { as a } \nearrow a^{*} .
$$

3. Any global maximal point $x_{a}$ of $\left|u_{a}\right|$ satisfies $\lim _{a \nearrow a^{*}} V_{\Omega}\left(x_{a}\right)=0$, and $w_{a}(x)$ satisfies

$$
\lim _{a \nearrow a^{*}} w_{a}(x)=\frac{w(x)}{\sqrt{a^{*}}} \quad \text { strongly in } H^{1}\left(\mathbb{R}^{2}, \mathbb{C}\right) .
$$

Proof. 1. On the contrary, suppose that $\varepsilon_{a} \nrightarrow 0$ as $a \nearrow a^{*}$. By the definition of $e_{F}(a)$, then we derive from (1.6) and the diamagnetic inequality (2.4) that

$$
e_{F}(a)=\int_{\mathbb{R}^{2}}\left|(\nabla-i \mathcal{A}) u_{a}\right|^{2} d x-\frac{a}{2} \int_{\mathbb{R}^{2}}\left|u_{a}\right|^{4} d x+\int_{\mathbb{R}^{2}} V_{\Omega}(x)\left|u_{a}\right|^{2} d x \geq \int_{\mathbb{R}^{2}} V_{\Omega}(x)\left|u_{a}\right|^{2} d x .
$$

Following the upper estimate of $e_{F}(a)$ in (3.1), we deduce from the above inequality that the minimizer $u_{a}$ is bounded uniformly in $\mathcal{H}$. Furthermore, since the embedding $\mathcal{H} \hookrightarrow L^{q}\left(\mathbb{R}^{2}, \mathbb{C}\right)$ is compact for $2 \leq q<\infty$, we obtain that there exists a subsequence of $\left\{u_{a}\right\}$, still denoted by $\left\{u_{a}\right\}$, such that

$$
u_{a} \rightarrow u_{0} \text { weakly in } \mathcal{H}, \quad u_{a} \rightarrow u_{0} \text { strongly in } L^{q}\left(\mathbb{R}^{2}, \mathbb{C}\right)
$$

for some $u_{0} \in \mathcal{H}$, where $q \in[2, \infty)$. Since $u_{a} \rightarrow u_{0}$ strongly in $L^{2}\left(\mathbb{R}^{2}, \mathbb{C}\right)$ as $a \nearrow a^{*}$, we now have $\left\|u_{0}\right\|_{2}^{2}=1$. However, it follows from (3.1) and (3.10) that

$$
0=\lim _{a \nearrow a^{*}} e_{F}(a) \geq \liminf _{a \nearrow a^{*}} \int_{\mathbb{R}^{2}} V_{\Omega}(x)\left|u_{a}\right|^{2} d x \geq \int_{\mathbb{R}^{2}} V_{\Omega}(x)\left|u_{0}\right|^{2} d x \geq 0,
$$

which implies that $u_{0} \equiv 0$ in $\mathbb{R}^{2}$ by the assumption $(V)$, a contradiction. Therefore, we have $\varepsilon_{a} \rightarrow 0$ as $a \nearrow a^{*}$.

We next prove that $\mu_{a} \varepsilon_{a}^{2} \rightarrow-1$ as $a \nearrow a^{*}$. Actually, since $0<\Omega<\Omega^{*}$ is fixed, the definition (1.9) of $\Omega^{*}$ implies that

$$
|x|^{2} \leq C(\Omega) V_{\Omega}(x) \quad \text { for sufficiently large }|x|>0 .
$$

By (3.12) we then obtain from (3.1) and (3.10) that for any given large constant $M>0$,

$$
\begin{aligned}
\int_{\mathbb{R}^{2}} \frac{\Omega^{2}|x|^{2}}{4}\left|u_{a}\right|^{2} d x & =\int_{|x| \leq M} \frac{\Omega^{2}|x|^{2}}{4}\left|u_{a}\right|^{2} d x+\int_{|x|>M} \frac{\Omega^{2}|x|^{2}}{4}\left|u_{a}\right|^{2} d x \\
& \leq C+C(\Omega) \int_{|x|>M} V_{\Omega}(x)\left|u_{a}\right|^{2} d x \leq C .
\end{aligned}
$$

It then follows from above that for any given $\sigma>0$,

$$
\begin{aligned}
\Omega\left|\int_{\mathbb{R}^{2}} x^{\perp} \cdot\left(i u_{a}, \nabla u_{a}\right) d x\right| & \leq \frac{\sigma}{2} \int_{\mathbb{R}^{2}}\left|\nabla u_{a}\right|^{2} d x+\frac{\Omega^{2}}{2 \sigma} \int_{\mathbb{R}^{2}}|x|^{2}\left|u_{a}\right|^{2} d x \\
& \leq \frac{\sigma}{2} \int_{\mathbb{R}^{2}}\left|\nabla u_{a}\right|^{2} d x+C(\sigma) .
\end{aligned}
$$


By the Gagliardo-Nirenberg inequality (1.6) and Lemma 3.1, we also have

$$
\limsup _{a \nearrow a^{*}} \frac{\frac{a}{2} \int_{\mathbb{R}^{2}}\left|u_{a}\right|^{4} d x-e_{F}(a)}{\int_{\mathbb{R}^{2}}\left|\nabla u_{a}\right|^{2} d x} \leq 1
$$

Suppose now that $\mu_{a} \varepsilon_{a}^{2} \nrightarrow-1$ as $a \nearrow a^{*}$. By taking a subsequence if necessary, we then obtain from above and (3.5) that there exists some constant $\gamma_{0}>0$ such that

$$
-\liminf _{a \nearrow a^{*}} \mu_{a} \varepsilon_{a}^{2}=\liminf _{a \nearrow a^{*}} \frac{\frac{a}{2} \int_{\mathbb{R}^{2}}\left|u_{a}\right|^{4} d x-e_{F}(a)}{\int_{\mathbb{R}^{2}}\left|\nabla u_{a}\right|^{2} d x} \leq 1-\gamma_{0}
$$

Applying (3.11)-(3.15), we thus deduce that

$$
\begin{aligned}
e_{F}(a) & =\int_{\mathbb{R}^{2}}\left|\nabla u_{a}\right|^{2} d x-\frac{a}{2} \int_{\mathbb{R}^{2}}\left|u_{a}\right|^{4} d x+\int_{\mathbb{R}^{2}} V(x)\left|u_{a}\right|^{2} d x-\Omega \int_{\mathbb{R}^{2}} x^{\perp} \cdot\left(i u_{a}, \nabla u_{a}\right) \\
& \geq \int_{\mathbb{R}^{2}}\left|\nabla u_{a}\right|^{2} d x-\frac{a}{2} \int_{\mathbb{R}^{2}}\left|u_{a}\right|^{4} d x-\frac{\sigma}{2} \int_{\mathbb{R}^{2}}\left|\nabla u_{a}\right|^{2} d x-C(\sigma) \\
& \geq\left(\gamma_{0}-\frac{\sigma}{2}\right) \int_{\mathbb{R}^{2}}\left|\nabla u_{a}\right|^{2} d x-C(\sigma)=\frac{\gamma_{0}}{2} \int_{\mathbb{R}^{2}}\left|\nabla u_{a}\right|^{2} d x-C\left(\gamma_{0}\right),
\end{aligned}
$$

where we take $\sigma=\gamma_{0}>0$ in the last identity. Since we have $\varepsilon_{a} \rightarrow 0$ as $a \nearrow a^{*}$, it follows from (3.6) that $\int_{\mathbb{R}^{2}}\left|\nabla u_{a}\right|^{2} d x \rightarrow \infty$ as $a \nearrow a^{*}$. The above inequality then yields that $e_{F}(a) \rightarrow \infty$ as $a \nearrow a^{*}$, which however contradicts to Lemma 3.1. This completes the proof of (1).

2. Denote $\bar{w}_{a}(x)=\varepsilon_{a} u_{a}\left(\varepsilon_{a} x+x_{a}\right) e^{-i\left(\frac{\varepsilon_{a} \Omega}{2} x \cdot x_{a}^{\perp}\right)}$ and $w_{a}(x):=\bar{w}_{a}(x) e^{i \theta_{a}}$, where the parameter $\theta_{a} \in[0,2 \pi)$ is chosen properly such that

$$
\left\|w_{a}-\frac{w}{\sqrt{a^{*}}}\right\|_{L^{2}\left(\mathbb{R}^{2}\right)}=\min _{\theta \in[0,2 \pi)}\left\|e^{i \theta} \bar{w}_{a}-\frac{w}{\sqrt{a^{*}}}\right\|_{L^{2}\left(\mathbb{R}^{2}\right)} .
$$

Rewrite $w_{a}(x)=R_{a}(x)+i I_{a}(x)$, where $R_{a}(x)$ denotes the real part of $w_{a}(x)$, and $I_{a}(x)$ denotes the imaginary part of $w_{a}(x)$. By (3.16), we obtain the following orthogonality condition

$$
\int_{\mathbb{R}^{2}} w(x) I_{a}(x) d x=0 .
$$

Following (3.4), we see that $w_{a}(x)$ satisfies the following Euler-Lagrange equation

$$
-\Delta w_{a}+i \varepsilon_{a}^{2} \Omega\left(x^{\perp} \cdot \nabla w_{a}\right)+\left[\frac{\varepsilon_{a}^{4} \Omega^{2}}{4}|x|^{2}+\varepsilon_{a}^{2} V_{\Omega}\left(\varepsilon_{a} x+x_{a}\right)-\varepsilon_{a}^{2} \mu_{a}-a\left|w_{a}\right|^{2}\right] w_{a}=0 \text { in } \mathbb{R}^{2},
$$

where $w_{a}$ is bounded uniformly in $H^{1}\left(\mathbb{R}^{2}, \mathbb{C}\right)$. Define $W_{a}(x)=\left|w_{a}(x)\right|^{2} \geq 0$ in $\mathbb{R}^{2}$. We then derive from (3.18) that

$$
\begin{aligned}
& -\frac{1}{2} \Delta W_{a}+\left|\nabla w_{a}\right|^{2}-\varepsilon_{a}^{2} \Omega x^{\perp} \cdot\left(i w_{a}, \nabla w_{a}\right) \\
& +\left[\frac{\varepsilon_{a}^{4} \Omega^{2}}{4}|x|^{2}+\varepsilon_{a}^{2} V_{\Omega}\left(\varepsilon_{a} x+x_{a}\right)-\varepsilon_{a}^{2} \mu_{a}-a W_{a}\right] W_{a}=0 \quad \text { in } \mathbb{R}^{2} .
\end{aligned}
$$

Since

$$
\left|\nabla w_{a}\right|^{2}-\varepsilon_{a}^{2} \Omega x^{\perp} \cdot\left(i w_{a}, \nabla w_{a}\right)+\frac{\varepsilon_{a}^{4} \Omega^{2}}{4}|x|^{2} W_{a} \geq 0 \text { in } \mathbb{R}^{2},
$$

which is due to the diamagnetic inequality (2.4), we have

$$
-\frac{1}{2} \Delta W_{a}-\varepsilon_{a}^{2} \mu_{a} W_{a}-a W_{a}^{2} \leq 0 \quad \text { in } \mathbb{R}^{2} .
$$

Since 0 is a global maximal point of $W_{a}(x)$ for all $a<a^{*}$, we have $-\Delta W_{a}(0) \geq 0$ for all $a<a^{*}$. Combining the fact that $\varepsilon_{a}^{2} \mu_{a} \rightarrow-1$ as $a \nearrow a^{*}$, we then get that $W_{a}(0) \geq \beta>0$ holds uniformly 
in $a$ for some positive constant $\beta$. Following De Giorgi-Nash-Moser theory [33, Theorem 4.1], we thus deduce from (3.20) that

$$
\int_{B_{2}(0)} W_{a}^{2} d x \geq C \max _{x \in B_{1}(0)} W_{a}(x) \geq C_{1}(\beta) \text { as } a \nearrow a^{*} .
$$

Moreover, since $w_{a}$ is bounded uniformly in $H^{1}\left(\mathbb{R}^{2}, \mathbb{C}\right)$, we obtain from $(3.21)$ that

$$
W_{a}(0)=\max _{x \in \mathbb{R}^{2}} W_{a}(x) \leq C \int_{B_{2}(0)} W_{a}^{2} d x \leq C .
$$

Combining (3.21) and (3.22), we get that

$$
\int_{B_{2}(0)}\left|w_{a}\right|^{2} d x \geq \int_{B_{2}(0)} \frac{W_{a}^{2}}{\max _{x \in \mathbb{R}^{2}} W_{a}(x)} d x \geq C_{2}(\beta)>0,
$$

which completes the proof of (3.8).

3. Since $\left|w_{a}\right|$ is bounded uniformly in $H^{1}\left(\mathbb{R}^{2}\right)$, we may assume that up to a subsequence if necessary, $\left|w_{a}\right|$ converges to $w_{0}$ weakly in $H^{1}\left(\mathbb{R}^{2}\right)$ as $a \nearrow a^{*}$ for some $0 \leq w_{0} \in H^{1}\left(\mathbb{R}^{2}\right)$. Note from (3.23) that $w_{0} \neq \equiv 0$ in $\mathbb{R}^{2}$. By the weak convergence, we may assume that $\left|w_{a}\right| \rightarrow w_{0}$ almost everywhere in $\mathbb{R}^{2}$ as $a \nearrow a^{*}$. Applying Brézis-Lieb lemma gives that

$$
\left\|w_{a}\right\|_{q}^{q}=\left\|w_{0}\right\|_{q}^{q}+\left\|\left|w_{a}\right|-w_{0}\right\|_{q}^{q}+o(1) \quad \text { as } a \nearrow a^{*}, \quad \text { where } 2 \leq q<\infty,
$$

and

$$
\left\|\nabla\left|w_{a}\right|\right\|_{2}^{2}=\left\|\nabla w_{0}\right\|_{2}^{2}+\left\|\nabla\left(\left|w_{a}\right|-w_{0}\right)\right\|_{2}^{2}+o(1) \quad \text { as } a \nearrow a^{*} .
$$

This also implies that $\left\|\left|w_{a}\right|-w_{0}\right\|_{2}^{2} \leq 1$ holds uniformly as $a \nearrow a^{*}$. Recall from (3.1) that $e_{F}(a) \rightarrow 0$ as $a \nearrow a^{*}$, and note also that

$$
1=-\lim _{a \nearrow a^{*}} \mu_{a} \varepsilon_{a}^{2}=\lim _{a \nearrow a^{*}} \frac{\frac{a}{2} \int_{\mathbb{R}^{2}}\left|u_{a}\right|^{4} d x-e_{F}(a)}{\int_{\mathbb{R}^{2}}\left|\nabla u_{a}\right|^{2} d x} .
$$

By the definition of $w_{a}(x)$, we therefore get that

$$
\lim _{a \nearrow a^{*}} \int_{\mathbb{R}^{2}}\left|w_{a}\right|^{4} d x=\frac{2}{a^{*}} .
$$

Thus, we have

$$
\begin{aligned}
& \lim _{a \nearrow a^{*}}\left\{\int_{\mathbb{R}^{2}}|\nabla| w_{a}||^{2} d x-\frac{a^{*}}{2} \int_{\mathbb{R}^{2}}\left|w_{a}\right|^{4} d x\right\} \\
& \leq \lim _{a \nearrow a^{*}}\left\{\int_{\mathbb{R}^{2}}\left|\nabla w_{a}\right|^{2} d x-\frac{a^{*}}{2} \int_{\mathbb{R}^{2}}\left|w_{a}\right|^{4} d x\right\}=0 .
\end{aligned}
$$

By the Gagliardo-Nirenberg inequality (1.6), we then get from (3.24), (3.25) and (3.27) that

$$
\begin{aligned}
0 \geq & \lim _{a \nearrow a^{*}}\left\{\int_{\mathbb{R}^{2}}|\nabla| w_{a}||^{2} d x-\frac{a^{*}}{2} \int_{\mathbb{R}^{2}}\left|w_{a}\right|^{4} d x\right\} \\
= & \int_{\mathbb{R}^{2}}\left|\nabla w_{0}\right|^{2} d x-\frac{a^{*}}{2} \int_{\mathbb{R}^{2}}\left|w_{0}\right|^{4} d x \\
& +\lim _{a \nearrow a^{*}}\left\{\int_{\mathbb{R}^{2}}\left|\nabla\left(\left|w_{a}\right|-w_{0}\right)\right|^{2} d x-\frac{a^{*}}{2} \int_{\mathbb{R}^{2}}|| w_{a}\left|-w_{0}\right|^{4} d x\right\} \\
\geq & \int_{\mathbb{R}^{2}}\left|\nabla w_{0}\right|^{2} d x-\frac{a^{*}}{2} \int_{\mathbb{R}^{2}}\left|w_{0}\right|^{4} d x \\
& +\frac{a^{*}}{2} \lim _{a \nearrow a^{*}}\left(||\left|w_{a}\right|-w_{0} \|_{2}^{-2}-1\right) \int_{\mathbb{R}^{2}}|| w_{a}\left|-w_{0}\right|^{4} d x \geq 0 .
\end{aligned}
$$


Using (1.6) again, the above inequality implies that $\left\|w_{0}\right\|_{2}^{2}=1$ and

$$
\left|w_{a}\right| \rightarrow w_{0} \text { strongly in } L^{2}\left(\mathbb{R}^{2}\right) \text { as } a \nearrow a^{*} .
$$

Due to the uniform boundedness of $\left|w_{a}\right|$ in $H^{1}\left(\mathbb{R}^{2}\right)$, we obtain that $\left|w_{a}\right| \rightarrow w_{0}$ strongly in $L^{4}\left(\mathbb{R}^{2}\right)$ as $a \nearrow a^{*}$. By the weak lower semicontinuity and (1.6), we further get that $\nabla\left|w_{a}\right| \rightarrow \nabla w_{0}$ strongly in $L^{2}\left(\mathbb{R}^{2}\right)$ as $a \nearrow a^{*}$. Therefore, $\sqrt{a^{*}} w_{0}$ must be an optimizer of the Gagliardo-Nirenberg inequality (1.6), and there exists $y_{0} \in \mathbb{R}^{2}$ such that up to a subsequence if necessary,

$$
\left|w_{a}(x)\right| \rightarrow w_{0}(x)=\frac{w\left(x+y_{0}\right)}{\sqrt{a^{*}}} \quad \text { strongly in } H^{1}\left(\mathbb{R}^{2}\right) \quad \text { as } a \nearrow a^{*} .
$$

Since the origin is a global maximal point of $\left|w_{a}\right|$ for all $a \in\left(0, a^{*}\right)$, it must be a global maximal point of $w\left(x+y_{0}\right)$, which implies that $y_{0}=0$, and up to a subsequence if necessary,

$$
\left|w_{a}(x)\right| \rightarrow \frac{w(x)}{\sqrt{a^{*}}} \quad \text { strongly in } H^{1}\left(\mathbb{R}^{2}\right) \text { as } a \nearrow a^{*} .
$$

Moreover, because the convergence (3.29) is independent of the subsequence $\left\{\left|w_{a}\right|\right\}$, we conclude that (3.29) holds for the whole sequence. Then it is not difficult to derive from (3.29) that up to a subsequence if necessary,

$$
\lim _{a \nearrow a^{*}} w_{a}=\frac{w}{\sqrt{a^{*}}} e^{i \sigma} \text { strongly in } H^{1}\left(\mathbb{R}^{2}, \mathbb{C}\right)
$$

for some $\sigma \in \mathbb{R}$. Moreover, we have $\sigma=0$ in view of (3.17). Since the convergence of (3.30) is independent of the choice of the subsequence, we deduce that (3.30) holds essentially true for the whole sequence, and hence (3.9) holds true.

Following (3.1), we also obtain that $\lim _{a \nearrow a^{*}} e_{F}(a)=0$, and hence $\lim _{a \nearrow a^{*}} V_{\Omega}\left(x_{a}\right)=0$ in view of (3.10) and (3.30), where $x_{a}$ is a global maximum point of $\left|u_{a}\right|$. This then completes the proof of Lemma 3.2 .

\section{1 $\quad L^{\infty}$-uniform convergence as $a \nearrow a^{*}$}

In this subsection, we first derive $L^{\infty}$-uniform convergence of $w_{a}(x)$ as $a \nearrow a^{*}$, based on which we finally finish the proof of Theorem 1.2.

Proposition 3.3. Under the assumptions of Theorem 1.2, let $u_{a}$ be a minimizer of $e_{F}(a)$, and consider the sequences $\left\{w_{a}\right\}$ and $\left\{x_{a}\right\}$ defined in Lemma 3.2. Then we have

(i). There exists a large constant $R>0$ such that as a $\nearrow a^{*}$,

$$
\left|w_{a}(x)\right| \leq C e^{-\frac{2}{3}|x|} \quad \text { in } \mathbb{R}^{2} / B_{R}(0) .
$$

(ii). The global maximal point $x_{a}$ of $\left|u_{a}\right|$ is unique as a $\nearrow a^{*}$, and $w_{a}(x)$ satisfies

$$
w_{a}(x) \rightarrow \frac{w(x)}{\sqrt{a^{*}}} \text { uniformly in } L^{\infty}\left(\mathbb{R}^{2}, \mathbb{C}\right) \text { as a } \nearrow a^{*} .
$$

(iii). The following estimate holds

$$
\Omega \int_{\mathbb{R}^{2}} x^{\perp}\left(i w_{a}(x), \nabla w_{a}(x)\right) d x=o\left(\varepsilon_{a}^{1+\frac{\gamma}{2}}\right) \text { as a } \nearrow a^{*},
$$

where $\varepsilon_{a}=\varepsilon_{a}>0$ is as in Lemma 3.2 and $\gamma=\min \{p, 2\}>0$. 
Proof. 1. Set $W_{a}=\left|w_{a}\right|^{2}$, so that $W_{a}$ satisfies (3.20). Recall from (3.29) that $W_{a} \rightarrow \frac{1}{a^{*}} w^{2}(|x|)$ strongly in $L^{2}\left(\mathbb{R}^{2}\right)$ as $a \nearrow a^{*}$. Applying De Giorgi-Nash-Moser theory [33, Theorem 4.1] to (3.20), then there exists a sufficiently large $R>0$ such that

$$
W_{a}(x) \leq \frac{1}{18 a^{*}} \text { in } \mathbb{R}^{2} / B_{R}(0)
$$

holds uniformly as $a \nearrow a^{*}$. By Lemma 3.2 , we then derive from (3.20) that for sufficiently large $R>0$,

$$
-\Delta W_{a}(x)+\frac{16}{9} W_{a}(x) \leq 0 \quad \text { in } \mathbb{R}^{2} / B_{R}(0)
$$

where we used that $-\varepsilon_{a}^{2} \mu_{a} \rightarrow 1$ as $a \nearrow a^{*}$. Applying the comparison principle to (3.35), the exponential decay (3.31) is then proved.

2. We first claim that $w_{a}(x)$ converges to $\frac{w(x)}{\sqrt{a^{*}}}$ uniformly in $L^{\infty}\left(\mathbb{R}^{2}, \mathbb{C}\right)$ as $a \nearrow a^{*}$. Indeed, by the exponential decay of (2.1) and (3.31), we only need to show the $L^{\infty}$-uniform convergence of $w_{a}(x)$ on any compact domain of $\mathbb{R}^{2}$ as $a \nearrow a^{*}$. Since $w_{a}(x)$ satisfies (3.18), denote

$$
G_{a}(x):=-i \varepsilon_{a}^{2} \Omega\left(x^{\perp} \cdot \nabla w_{a}\right)-\left[\frac{\varepsilon_{a}^{4} \Omega^{2}}{4}|x|^{2}+\varepsilon_{a}^{2} V_{\Omega}\left(\varepsilon_{a} x+x_{a}\right)-\varepsilon_{a}^{2} \mu_{a}-a\left|w_{a}\right|^{2}\right] w_{a},
$$

so that

$$
-\Delta w_{a}(x)=G_{a}(x) \quad \text { in } H^{1}\left(\mathbb{R}^{2}, \mathbb{C}\right) .
$$

Because $w_{a}(x)$ is bounded uniformly in $H^{1}\left(\mathbb{R}^{2}, \mathbb{C}\right)$ as $a \nearrow a^{*}, G_{a}(x)$ is also bounded uniformly in $L_{\text {loc }}^{2}\left(\mathbb{R}^{2}, \mathbb{C}\right)$ as $a \nearrow a^{*}$. For any large $R>0$, it then follows from [25, Theorem 8.8] that

$$
\left\|w_{a}(x)\right\|_{H^{2}\left(B_{R}\right)} \leq C\left(\left\|w_{a}(x)\right\|_{H^{1}\left(B_{R+1}\right)}+\left\|G_{a}(x)\right\|_{L^{2}\left(B_{R+1}\right)}\right),
$$

where $C>0$ is independent of $a>0$ and $R>0$. Therefore, $w_{a}(x)$ is also bounded uniformly in $H_{\text {loc }}^{2}\left(\mathbb{R}^{2}, \mathbb{C}\right)$. Since the embedding $H^{2}\left(B_{R}\right) \hookrightarrow L^{\infty}\left(B_{R}\right)$ is compact, cf. [25, Theorem 7.26], we obtain that there exists a subsequence $\left\{w_{a_{k}}(x)\right\}$ of $\left\{w_{a}(x)\right\}$ such that

$$
\lim _{a_{k} \nearrow a^{*}} w_{a_{k}}(x)=w_{0}(x) \quad \text { uniformly in } L^{\infty}\left(B_{R}, \mathbb{C}\right) .
$$

Since $R>0$ is arbitrary, we get from (3.9) that

$$
\lim _{a_{k} \nearrow a^{*}} w_{a_{k}}(x)=\frac{w}{\sqrt{a^{*}}} \text { uniformly in } L_{l o c}^{\infty}\left(\mathbb{R}^{2}, \mathbb{C}\right) .
$$

Because the above convergence is independent of the subsequence that we choose, (3.38) holds essentially for the whole sequence. This further implies that $(3.32)$ holds uniformly in $L^{\infty}\left(\mathbb{R}^{2}, \mathbb{C}\right)$ as $a \nearrow a^{*}$.

We next prove the uniqueness of $x_{a}$ as $a \nearrow a^{*}$, where $x_{a}$ is a global maximum point of $\left|u_{a}\right|$. By (3.37), one can get that $\left|\nabla w_{a}\right|$ is bounded uniformly in $L_{l o c}^{q}\left(\mathbb{R}^{2}\right)$ as $a \nearrow a^{*}$ for any $q \geq 2$. The $L^{p}$ estimate [25, Theorem 9.11] applied to (3.36) then implies that $w_{a}$ is bounded uniformly in $W_{l o c}^{2, q}\left(\mathbb{R}^{2}\right)$ as a $\nearrow a^{*}$. The standard Sobolev embedding thus gives that $w_{a}$ is bounded uniformly in $C_{l o c}^{1, \alpha}\left(\mathbb{R}^{2}\right)$. Furthermore, since $\varepsilon_{a}^{2} V_{\Omega}\left(\varepsilon_{a} x+x_{a}\right) \in C_{l o c}^{\alpha}\left(\mathbb{R}^{2}\right)$, it follows from the Schauder estimate [25, Theorem 6.2] that $w_{a}$ is bounded uniformly in $C_{l o c}^{2, \alpha}\left(\mathbb{R}^{2}\right)$ as $a \nearrow a^{*}$. Hence, there exists $w_{0} \in C_{l o c}^{2}\left(\mathbb{R}^{2}\right)$ such that

$$
w_{a} \rightarrow w_{0} \text { in } C_{l o c}^{2}\left(\mathbb{R}^{2}\right) \text { as } a \nearrow a^{*} .
$$

Noting from (3.32) that $w_{a} \rightarrow \frac{w}{\sqrt{a^{*}}}$ in $L^{\infty}\left(\mathbb{R}^{2}\right)$ as $a \nearrow a^{*}$, we conclude that $w_{0} \equiv \frac{w}{\sqrt{a^{*}}}$, and hence

$$
\left|w_{a}\right| \rightarrow \frac{w}{\sqrt{a^{*}}} \text { in } C_{l o c}^{2}\left(\mathbb{R}^{2}\right) \text { as } a \nearrow a^{*}
$$


Because the origin is the unique global maximum point of $w$, the above convergence shows that all global maximum points of $\left|w_{a}\right|$ must stay in a small ball $B_{\delta}(0)$ as $a \nearrow a^{*}$ for some small $\delta>0$. Since $w^{\prime \prime}(0)<0$, we conclude that $w^{\prime \prime}(r)<0$ for $0 \leq r<\delta$. It then follows from [48, Lemma $4.2]$ that as $a \nearrow a^{*}$, each $\left|w_{a}\right|$ has a unique global maximum point which is just the origin. This further proves the uniqueness of global maximum points for $\left|u_{a}\right|$ as $a \nearrow a^{*}$.

3. By the definition of $e_{F}(a)$, we obtain from Lemma 3.2 that

$$
\begin{aligned}
e_{F}(a)= & F_{a}\left(u_{a}\right)=F_{a}\left(\frac{1}{\varepsilon_{a}} e^{i\left(\frac{\Omega}{2} x \cdot x_{a}^{\perp}-\theta\right)} w_{a}\left(\frac{x-x_{a}}{\varepsilon_{a}}\right)\right) \\
= & \frac{1}{\varepsilon_{a}^{2}} \int_{\mathbb{R}^{2}}\left|\nabla w_{a}(x)\right|^{2} d x-\frac{a}{2 \varepsilon_{a}^{2}} \int_{\mathbb{R}^{2}}\left|w_{a}(x)\right|^{4} d x+\frac{\varepsilon_{a}^{2} \Omega^{2}}{4} \int_{\mathbb{R}^{2}}|x|^{2}\left|w_{a}(x)\right|^{2} d x \\
& +\int_{\mathbb{R}^{2}} V_{\Omega}\left(\varepsilon_{a} x+x_{a}\right)\left|w_{a}(x)\right|^{2} d x-\Omega \int_{\mathbb{R}^{2}} x^{\perp} \cdot\left(i w_{a}(x), \nabla w_{a}(x)\right) d x .
\end{aligned}
$$

Applying the convergence (3.32) and the exponential decay (3.31), we have

$$
\begin{aligned}
\Omega \int_{\mathbb{R}^{2}} x^{\perp} \cdot\left(i w_{a}(x), \nabla w_{a}(x)\right) d x & =\Omega \int_{\mathbb{R}^{2}} x^{\perp} \cdot\left(R_{a} \nabla I_{a}-I_{a} \nabla R_{a}\right) d x \\
& =2 \Omega \int_{\mathbb{R}^{2}} x^{\perp} \cdot\left(R_{a} \nabla I_{a}\right) d x \leq C\left\|\nabla I_{a}\right\|_{L^{2}},
\end{aligned}
$$

where $w_{a}(x):=R_{a}(x)+i I_{a}(x)$ is defined after (3.16). We then derive from above that

$$
\varepsilon_{a}^{2} e_{F}(a) \geq \int_{\mathbb{R}^{2}}\left(\left|\nabla R_{a}\right|^{2}+\left|\nabla I_{a}\right|^{2}\right) d x-\frac{a^{*}}{2} \int_{\mathbb{R}^{2}}\left(R_{a}^{4}+I_{a}^{4}+2 R_{a}^{2} I_{a}^{2}\right) d x-C \varepsilon_{a}^{2}\left\|\nabla I_{a}\right\|_{L^{2}} .
$$

Since it follows from (3.32) that $R_{a} \rightarrow \frac{w}{\sqrt{a^{*}}}$ and $I_{a} \rightarrow 0$ uniformly in $\mathbb{R}^{2}$ as $a \nearrow a^{*}$, we now have

$$
\begin{aligned}
& \int_{\mathbb{R}^{2}}\left|\nabla R_{a}\right|^{2} d x-\frac{a^{*}}{2} \int_{\mathbb{R}^{2}}\left(R_{a}^{4}+I_{a}^{4}+2 R_{a}^{2} I_{a}^{2}\right) d x \\
\geq & \int_{\mathbb{R}^{2}}\left|\nabla R_{a}\right|^{2} d x\left(1-\int_{\mathbb{R}^{2}}\left|R_{a}\right|^{2} d x\right)-\frac{a^{*}}{2} \int_{\mathbb{R}^{2}}\left(I_{a}^{2}+2 R_{a}^{2}\right) I_{a}^{2} d x \\
= & (1+o(1)) \int_{\mathbb{R}^{2}} I_{a}^{2} d x-(1+o(1)) \int_{\mathbb{R}^{2}} w^{2} I_{a}^{2} d x \text { as } a \nearrow a^{*},
\end{aligned}
$$

where the Gagliardo-Nirenberg inequality (1.6) is used. Then, we derive from (3.40) that

$$
\begin{aligned}
\varepsilon_{a}^{2} e_{F}(a) & \geq \int_{\mathbb{R}^{2}}\left|\nabla I_{a}\right|^{2} d x+\int_{\mathbb{R}^{2}} I_{a}^{2} d x-\int_{\mathbb{R}^{2}} w^{2} I_{a}^{2} d x-o(1) \int_{\mathbb{R}^{2}} I_{a}^{2} d x-C \varepsilon_{a}^{2}\left\|\nabla I_{a}\right\|_{L^{2}} \\
& =\left(\mathcal{L} I_{a}, I_{a}\right)-o(1) \int_{\mathbb{R}^{2}} I_{a}^{2} d x-C \varepsilon_{a}^{2}\left\|\nabla I_{a}\right\|_{L^{2}} \quad \text { as } a \nearrow a^{*},
\end{aligned}
$$

where the operator $\mathcal{L}$ is defined by

$$
\mathcal{L}:=-\Delta-w^{2}+1 \quad \text { in } L^{2}\left(\mathbb{R}^{2}\right) .
$$

Note from [42, Corollary 11.9 and Theorem 11.8] that $(0, w)$ is the first eigenpair of $\mathcal{L}$ and $\operatorname{ker}(\mathcal{L})=\{w\}$. Moreover, since the essential spectrum of the operator $\mathcal{L}$ satisfies $\sigma_{\text {ess }}(\mathcal{L})=$ $[1,+\infty)$, we have $0 \in \sigma_{d}(\mathcal{L})$, where $\sigma_{d}$ denotes its discrete spectrum. Because $I_{a}$ is orthogonal to $w(x)$ by (3.17), we deduce that

$$
\left(\mathcal{L} I_{a}, I_{a}\right) \geq\left\|I_{a}\right\|_{L^{2}\left(\mathbb{R}^{2}\right)}^{2} \text { as } a \nearrow a^{*} .
$$

On the other hand, we obtain from (3.42) that

$$
\left(\mathcal{L} I_{a}, I_{a}\right) \geq\left\|\nabla I_{a}\right\|_{L^{2}\left(\mathbb{R}^{2}\right)}^{2}-\|w\|_{L^{\infty}}^{2}\left\|I_{a}\right\|_{L^{2}\left(\mathbb{R}^{2}\right)}^{2} .
$$


We thus deduce from (3.43) and (3.44) that there exists a constant $\rho>0$ such that as $a \nearrow a^{*}$,

$$
\left(\mathcal{L} I_{a}, I_{a}\right) \geq \rho\left\|I_{a}\right\|_{H^{1}\left(\mathbb{R}^{2}\right)}^{2} .
$$

Inserting (3.45) into (3.41), we have

$$
\varepsilon_{a}^{2} e_{F}(a) \geq \frac{\rho}{2}\left\|I_{a}\right\|_{H^{1}\left(\mathbb{R}^{2}\right)}^{2}-C \varepsilon_{a}^{2}\left\|\nabla I_{a}\right\|_{L^{2}\left(\mathbb{R}^{2}\right)} \quad \text { as } a \nearrow a^{*} .
$$

Similar to (3.10), applying Lemma 3.1 yields that for $\gamma=\min \{p, 2\}>0$,

$$
\begin{aligned}
& M\left(a^{*}-a\right)^{\frac{\gamma}{\gamma+2}} \geq e_{F}(a) \\
= & \int_{\mathbb{R}^{2}}\left|(\nabla-i \mathcal{A}) u_{a}\right|^{2} d x-\frac{a}{2} \int_{\mathbb{R}^{2}}\left|u_{a}\right|^{4} d x+\int_{\mathbb{R}^{2}} V_{\Omega}(x)\left|u_{a}\right|^{2} d x \\
\geq & \int_{\mathbb{R}^{2}}|\nabla| u_{a}||^{2} d x-\frac{a}{2} \int_{\mathbb{R}^{2}}\left|u_{a}\right|^{4} d x \geq\left(1-\frac{a}{a^{*}}\right) \int_{\mathbb{R}^{2}}|\nabla| u_{a}||^{2} d x \\
\geq & \frac{1}{2}\left(1-\frac{a}{a^{*}}\right) \varepsilon_{a}^{-2} \text { as } a \nearrow a^{*} .
\end{aligned}
$$

This further implies that

$$
\left(a^{*}-a\right)^{\frac{1}{\gamma+2}} \leq C \varepsilon_{a}, \quad e_{F}(a) \leq M\left(a^{*}-a\right)^{\frac{\gamma}{\gamma+2}} \leq C \varepsilon_{a}^{\gamma} \quad \text { as } a \nearrow a^{*} .
$$

Following (3.46) and (3.47), we obtain that

$$
\left\|I_{a}\right\|_{H^{1}\left(\mathbb{R}^{2}\right)} \leq C \varepsilon_{a}^{1+\frac{\gamma}{2}} \text { as } a \nearrow a^{*} .
$$

Applying (3.31) and (3.48) now yields that

$$
\begin{aligned}
& \int_{\mathbb{R}^{2}} x^{\perp} \cdot\left(i w_{a}(x), \nabla w_{a}(x)\right) d x \\
= & 2 \int_{\mathbb{R}^{2}} x^{\perp} \cdot\left(R_{a} \nabla I_{a}\right) d x=2 \int_{\mathbb{R}^{2}} x^{\perp} \cdot\left(\frac{w(x)}{\sqrt{a^{*}}} \nabla I_{a}\right) d x+o\left(\varepsilon_{a}^{1+\frac{\gamma}{2}}\right) \\
= & -2 \int_{\mathbb{R}^{2}} x^{\perp} \cdot\left(I_{a} \nabla \frac{w(x)}{\sqrt{a^{*}}}\right) d x+o\left(\varepsilon_{a}^{1+\frac{\gamma}{2}}\right)=o\left(\varepsilon_{a}^{1+\frac{\gamma}{2}}\right) \text { as } a \nearrow a^{*},
\end{aligned}
$$

where the convergence (3.32) is also used. The proof of Proposition 3.3 is therefore complete.

Completion of the proof for Theorem 1.2. In view of (3.7) and (3.32), to establish Theorem 1.2 , the rest is to prove that

$$
\varepsilon_{a}:=\left(\int_{\mathbb{R}^{2}}\left|\nabla u_{a}\right|^{2} d x\right)^{-\frac{1}{2}}=\frac{\left(a^{*}-a\right)^{\frac{1}{2+\gamma}}}{\lambda}+o\left(\left(a^{*}-a\right)^{\frac{1}{2+\gamma}}\right)>0 \text { as } a \nearrow a^{*},
$$

where $\gamma=\min \{2, p\}>0$ and $\lambda>0$ is as in (1.16).

In order to prove (3.50), we take

$$
u_{\beta}(x)=\frac{\beta}{\|w\|_{2}\left(a^{*}-a\right)^{\frac{1}{2+\gamma}}} w\left(\frac{\beta}{\left(a^{*}-a\right)^{\frac{1}{2+\gamma}}} x-y_{0}\right) e^{\frac{i \Omega y_{0}^{\perp} \cdot x}{2 \tau}},
$$

where $\beta \in(0, \infty)$ is to be determined later, as a test function of the energy $e_{F}(a)$ as $a \nearrow a^{*}$, and minimize it over $\beta>0$. By the similar calculations of Lemma 3.1, one then gets that

$$
e_{F}(a) \leq \min _{\beta \in(0, \infty)} F_{a}\left(u_{\beta}\right)=\left(1+\frac{2}{\gamma}\right) \frac{\lambda^{2}}{a^{*}}\left(a^{*}-a\right)^{\gamma /(2+\gamma)} \quad \text { as } a \nearrow a^{*} .
$$


On the other hand, following (3.39) and (3.33), we derive from Lemma 3.2 that

$$
\begin{aligned}
e_{F}(a)= & F_{a}\left(u_{a}\right) \\
= & \frac{1}{\varepsilon_{a}^{2}}\left[\int_{\mathbb{R}^{2}}\left|\nabla w_{a}(x)\right|^{2} d x-\frac{a^{*}}{2} \int_{\mathbb{R}^{2}}\left|w_{a}(x)\right|^{4} d x\right]+\frac{a^{*}-a}{2 \varepsilon_{a}^{2}} \int_{\mathbb{R}^{2}}\left|w_{a}(x)\right|^{4} d x \\
& +\frac{\varepsilon_{a}^{2} \Omega^{2}}{4} \int_{\mathbb{R}^{2}}|x|^{2}\left|w_{a}(x)\right|^{2} d x+\int_{\mathbb{R}^{2}} V_{\Omega}\left(\varepsilon_{a} x+x_{a}\right)\left|w_{a}(x)\right|^{2} d x \\
& -\Omega \int_{\mathbb{R}^{2}} x^{\perp}\left(i w_{a}(x), \nabla w_{a}(x)\right) d x .
\end{aligned}
$$

The term in the square bracket is non-negative and can be dropped for a lower bound of $e_{F}(a)$. Moreover, we infer from (3.33) that as $a \nearrow a^{*}$,

$$
\Omega \int_{\mathbb{R}^{2}} x^{\perp}\left(i w_{a}(x), \nabla w_{a}(x)\right) d x= \begin{cases}o\left(\varepsilon_{a}^{p}\right), & \text { if } 0<p \leq 2 \\ o\left(\varepsilon_{a}^{2}\right), & \text { if } p>2 .\end{cases}
$$

Because $w_{a} \rightarrow \frac{w}{\sqrt{a^{*}}}$ uniformly in $\mathbb{R}^{2}$ as $a \nearrow a^{*}$, we get from the exponential decay (3.31) that

$$
\begin{gathered}
\frac{a^{*}-a}{2 \varepsilon_{a}^{2}} \int_{\mathbb{R}^{2}}\left|w_{a}(x)\right|^{4} d x=[1+o(1)] \frac{a^{*}-a}{2\left(a^{*}\right)^{2} \varepsilon_{a}^{2}} \int_{\mathbb{R}^{2}}|w|^{4} d x \text { as } a \nearrow a^{*}, \\
\frac{\varepsilon_{a}^{2} \Omega^{2}}{4} \int_{\mathbb{R}^{2}}|x|^{2}\left|w_{a}(x)\right|^{2} d x=[1+o(1)] \frac{\varepsilon_{a}^{2} \Omega^{2}}{4 a^{*}} \int_{\mathbb{R}^{2}}|x|^{2}|w(x)|^{2} d x \text { as } a \nearrow a^{*},
\end{gathered}
$$

and

$$
\begin{aligned}
& \int_{\mathbb{R}^{2}} V_{\Omega}\left(\varepsilon_{a} x+x_{a}\right)\left|w_{a}(x)\right|^{2} d x \\
= & \int_{B \frac{1}{\sqrt{\varepsilon_{a}}}} V_{\Omega}\left(\varepsilon_{a} x+x_{a}\right)\left|w_{a}(x)\right|^{2} d x+\int_{\mathbb{R}^{2} / B \frac{1}{\sqrt{\varepsilon_{a}}}} V_{\Omega}\left(\varepsilon_{a} x+x_{a}\right)\left|w_{a}(x)\right|^{2} d x \\
= & \varepsilon_{a}^{p} \int_{B \frac{1}{\sqrt{\varepsilon_{a}}}} h\left(x+\varepsilon_{a}^{-1} x_{a}\right)\left|w_{a}(x)\right|^{2} d x+o\left(\varepsilon_{a}^{p}\right) \\
= & {[1+o(1)] \frac{\varepsilon_{a}^{p}}{a^{*}} \int_{\mathbb{R}^{2}} h\left(x+\varepsilon_{a}^{-1} x_{a}\right)|w(x)|^{2} d x \text { as } a \nearrow a^{*} . }
\end{aligned}
$$

Note that for $p=2$,

$$
\begin{aligned}
& \frac{a^{*}-a}{2\left(a^{*}\right)^{2} \varepsilon_{a}^{2}} \int_{\mathbb{R}^{2}}|w|^{4} d x+\frac{\varepsilon_{a}^{2} \Omega^{2}}{4 a^{*}} \int_{\mathbb{R}^{2}}|x|^{2}|w(x)|^{2} d x+\frac{\varepsilon_{a}^{p}}{a^{*}} \int_{\mathbb{R}^{2}} h\left(x+\varepsilon_{a}^{-1} x_{a}\right)|w(x)|^{2} d x \\
\geq & \left(1+\frac{2}{\gamma}\right) \frac{\lambda^{2}}{a^{*}}\left(a^{*}-a\right)^{\gamma /(2+\gamma)} \text { as } a \nearrow a^{*},
\end{aligned}
$$

where the identity holds if and only if (3.50) holds true. Applying (3.51), we then conclude from above that for $p=2$,

$$
e_{F}(a) \approx\left(1+\frac{2}{\gamma}\right) \frac{\lambda^{2}}{a^{*}}\left(a^{*}-a\right)^{\gamma /(2+\gamma)} \text { as } a \nearrow a^{*},
$$

and (3.50) is hence true for $p=2$. Similarly, one can also obtain from above that (3.50) holds true for the cases where $p \neq 2$. This completes the proof of Theorem 1.2.

The proof of Theorem 1.2 implies, see those around (3.53), that for all $p>0$,

$$
\varepsilon_{a}:=\left(\int_{\mathbb{R}^{2}}\left|\nabla u_{a}\right|^{2} d x\right)^{-\frac{1}{2}}=\frac{\left(a^{*}-a\right)^{\frac{1}{2+\gamma}}}{\lambda}+o\left(\left(a^{*}-a\right)^{\frac{1}{2+\gamma}}\right)>0 \text { as } a \nearrow a^{*},
$$




$$
0<\frac{1}{\varepsilon_{a}^{2}}\left[\int_{\mathbb{R}^{2}}\left|\nabla w_{a}(x)\right|^{2} d x-\frac{a^{*}}{2} \int_{\mathbb{R}^{2}}\left|w_{a}(x)\right|^{4} d x\right]=o\left(\varepsilon_{a}^{\gamma}\right) \text { as } a \nearrow a^{*}
$$

and

$$
\lim _{a \nearrow a^{*}} \frac{x_{a}}{\varepsilon_{a}}=y_{0}, \text { if } 0<p \leq 2,
$$

where $y_{0}$ is a global minimum point of $H(y)=\int_{\mathbb{R}^{2}} h(x+y) w^{2}(x) d x$ defined in the assumption $(V)$.

\section{Refined Limiting Profiles}

In this section we always assume $0<\Omega<\Omega^{*}$, where $\Omega^{*}>0$ is defined as in (1.9), and suppose $V(x) \in L_{\mathrm{loc}}^{\infty}\left(\mathbb{R}^{2}\right)$ satisfying (1.8) and

$\left(V_{1}\right) . V_{\Omega}(x)=V(x)-\frac{\Omega^{2}}{4}|x|^{2} \geq 0, V_{\Omega}(x) \in C^{2}\left(\mathbb{R}^{2}\right)$ is a homogeneous function of degree 2 and $H(y)=\int_{\mathbb{R}^{2}} V_{\Omega}(x+y) w^{2}(x) d x$ admits a unique critical point $y_{0} \in \mathbb{R}^{2}$.

It is not difficult to get from the above assumption that Theorem 1.2 is applicable as $a \nearrow a^{*}$. As before, let $u_{a}$ be a minimizer of $e_{F}(a)$ as $a \nearrow a^{*}$, and set

$$
\begin{aligned}
w_{a}(x) & :=\varepsilon_{a} u_{a}\left(\varepsilon_{a} x+x_{a}\right) e^{-i\left(\frac{\varepsilon_{a} \Omega}{2} x \cdot x_{a}^{\perp}-\theta_{a}\right)} \\
& :=R_{a}(x)+i I_{a}(x)=\frac{w(x)}{\sqrt{a^{*}}}+\hat{R}_{a}(x)+i I_{a}(x), \quad \varepsilon_{a}:=\left(\int_{\mathbb{R}^{2}}\left|\nabla u_{a}\right|^{2} d x\right)^{-\frac{1}{2}}>0,
\end{aligned}
$$

where $\theta_{a} \in[0,2 \pi)$ is chosen properly such that (3.16) holds, and $x_{a} \in \mathbb{R}^{2}$ denotes the unique global maximal point of $\left|u_{a}\right|$ as $a \nearrow a^{*}$. Here $R_{a}, \hat{R}_{a}$ and $I_{a}$ are real-valued functions in $H^{1}\left(\mathbb{R}^{2}\right)$. Following the proof of Theorem 1.2, we reduce from (3.17) and (3.32) that

$$
\int_{\mathbb{R}^{2}} w I_{a} d x \equiv 0, \quad \hat{R}_{a} \rightarrow 0 \text { and } I_{a} \rightarrow 0 \text { uniformly in } \mathbb{R}^{2} \text { as } a \nearrow a^{*} .
$$

Based on (4.2), the main purpose of this section is to establish the following refined estimates of $\hat{R}_{a}$ and $I_{a}$ as $a \nearrow a^{*}$.

Proposition 4.1. Suppose $V(x) \in L_{\mathrm{loc}}^{\infty}\left(\mathbb{R}^{2}\right)$ satisfies (1.8) and $\left(V_{1}\right)$, and assume $0<\Omega<\Omega^{*}$, where $\Omega^{*}>0$ is defined as in (1.9). Suppose $u_{a}$ is a minimizer of $e_{F}(a)$, and let $\hat{R}_{a}$ and $I_{a}$ be defined by (4.1). Then there exists a constant $C>0$, independent of $0<a<a^{*}$, such that as $a \nearrow a^{*}$,

(i). $\left|\hat{R}_{a}(x)\right|,\left|\nabla \hat{R}_{a}(x)\right| \leq C \varepsilon_{a}^{4} e^{-\frac{1}{2}|x|}$ in $\mathbb{R}^{2}$,

(ii). $\left|I_{a}(x)\right|,\left|\nabla I_{a}(x)\right| \leq C \varepsilon_{a}^{6} e^{-\frac{1}{8}|x|}$ in $\mathbb{R}^{2}$,

where $\varepsilon_{a}>0$ is as in (4.1).

In order to prove Proposition 4.1, we first give some observations. Because $\nabla\left|w_{a}(0)\right| \equiv 0$ holds for all $a \in\left(0, a^{*}\right)$, we calculate from (4.1) that

$$
\nabla \hat{R}_{a}(0)=-\frac{I_{a}(0) \nabla I_{a}(0)}{\frac{w(0)}{\sqrt{a^{*}}}+\hat{R}_{a}(0)} \rightarrow 0 \text { uniformly in } \mathbb{R}^{2} \text { as a } \nearrow a^{*} .
$$

Recall from (3.18) that $w_{a}$ satisfies the following equation

$$
\Delta w_{a}-i \varepsilon_{a}^{2} \Omega\left(x^{\perp} \cdot \nabla w_{a}\right)-\left[\frac{\varepsilon_{a}^{4} \Omega^{2}}{4}|x|^{2}+\varepsilon_{a}^{2} V_{\Omega}\left(\varepsilon_{a} x+x_{a}\right)-\mu_{a} \varepsilon_{a}^{2}-a\left|w_{a}\right|^{2}\right] w_{a}=0 \text { in } \mathbb{R}^{2},
$$


where $\varepsilon_{a}>0$ is as in (4.1), and $\mu_{a} \in \mathbb{R}$ is a Lagrange multiplier. Note from Lemma 3.2 that $\mu_{a} \in \mathbb{R}$ satisfies $\mu_{a} \varepsilon_{a}^{2} \rightarrow-1$ as $a \nearrow a^{*}$. For simplicity, we denote the operator $\mathcal{L}_{a}$ by

$$
\mathcal{L}_{a}:=-\Delta+\left(\frac{\varepsilon_{a}^{4} \Omega^{2}}{4}|x|^{2}+\varepsilon_{a}^{2} V_{\Omega}\left(\varepsilon_{a} x+x_{a}\right)-\varepsilon_{a}^{2} \mu_{a}-a\left|w_{a}\right|^{2}\right) .
$$

It then follows from (4.4) that $\left(R_{a}, I_{a}\right)$ satisfies the following system

$$
\left\{\begin{aligned}
\mathcal{L}_{a} R_{a} & =\varepsilon_{a}^{2} \Omega\left(x^{\perp} \cdot \nabla I_{a}\right) \text { in } \mathbb{R}^{2}, \\
\mathcal{L}_{a} I_{a} & =-\varepsilon_{a}^{2} \Omega\left(x^{\perp} \cdot \nabla R_{a}\right) \text { in } \mathbb{R}^{2} .
\end{aligned}\right.
$$

By applying Lemma A.1, we start with the following estimates:

Lemma 4.2. Under the assumptions of Proposition 4.1, we have

1. There exists a constant $C>0$, independent of $0<a<a^{*}$, such that as a $\nearrow a^{*}$,

$$
\left|\nabla R_{a}(x)\right|,\left|\nabla I_{a}(x)\right| \leq C e^{-\frac{2}{3}|x|} \text { in } \mathbb{R}^{2},
$$

where $R_{a}$ and $I_{a}$ are defined by (4.1).

2. The Lagrange multiplier $\mu_{a}$ of (4.4) satisfies

$$
\left|1+\mu_{a} \varepsilon_{a}^{2}\right| \leq C \varepsilon_{a}^{4} \text { as a } \nearrow a^{*},
$$

where $\varepsilon_{a}>0$ is as in (4.1) and $C>0$ is independent of $0<a<a^{*}$.

Proof. 1. We first claim that

$$
\left\|\nabla w_{a}\right\|_{L^{\infty}\left(\mathbb{R}^{2}\right)} \leq C \text { and } \lim _{|x| \rightarrow \infty}\left|\nabla w_{a}(x)\right|=0 \text { as } a \nearrow a^{*},
$$

where $C>0$ is independent of $0<a<a^{*}$. To prove the above claim, denote

$$
\hat{V}_{a}(x)=\frac{\varepsilon_{a}^{4} \Omega^{2}}{4}|x|^{2}+\varepsilon_{a}^{2} V_{\Omega}\left(\varepsilon_{a} x+x_{a}\right)-\mu_{a} \varepsilon_{a}^{2}-a\left|w_{a}\right|^{2},
$$

where $\mu_{a} \varepsilon_{a}^{2} \rightarrow-1$ as $a \nearrow a^{*}$. We then derive from (4.4) that $w_{a}$ satisfies

$$
\left[-\Delta+\hat{V}_{a}(x)\right] w_{a}+i \varepsilon_{a}^{2} \Omega\left(x^{\perp} \cdot \nabla w_{a}\right)=0 \text { in } \mathbb{R}^{2} .
$$

Following (4.11), we obtain that for $i \neq j$,

$$
\left[-\Delta+\hat{V}_{a}(x)\right] \partial_{i} w_{a}+\partial_{i} \hat{V}_{a}(x) w_{a}+i \varepsilon_{a}^{2} \Omega\left(x^{\perp} \cdot \nabla \partial_{i} w_{a}\right)+(-1)^{i+1} i \varepsilon_{a}^{2} \Omega \partial_{j} w_{a}=0 \text { in } \mathbb{R}^{2},
$$

which further implies that for $i \neq j$,

$$
\begin{aligned}
& -\frac{1}{2} \Delta\left|\partial_{i} w_{a}\right|^{2}+\hat{V}_{a}(x)\left|\partial_{i} w_{a}\right|^{2}+\left|\nabla \partial_{i} w_{a}\right|^{2}+\partial_{i} \hat{V}_{a}(x)\left(w_{a}, \partial_{i} w_{a}\right) \\
& -\varepsilon_{a}^{2} \Omega x^{\perp} \cdot\left(i \partial_{i} w_{a}, \nabla \partial_{i} w_{a}\right)+(-1)^{i+1} \varepsilon_{a}^{2} \Omega\left(i \partial_{j} w_{a}, \partial_{i} w_{a}\right)=0 \text { in } \mathbb{R}^{2} .
\end{aligned}
$$

Note from (3.31) and (4.10) that for $i \neq j$,

$$
\begin{gathered}
\varepsilon_{a}^{2} \Omega x^{\perp} \cdot\left(i \partial_{i} w_{a}, \nabla \partial_{i} w_{a}\right) \leq \frac{\varepsilon_{a}^{4} \Omega^{2}}{4}|x|^{2}\left|\partial_{i} w_{a}\right|^{2}+\left|\nabla \partial_{i} w_{a}\right|^{2}, \\
\partial_{i} \hat{V}_{a}(x)\left(w_{a}, \partial_{i} w_{a}\right) \leq\left|\partial_{i} \hat{V}_{a}(x)\right|^{2}\left|w_{a}\right|^{2}+\frac{1}{4}\left|\partial_{i} w_{a}\right|^{2} \leq C \varepsilon_{a}^{4} e^{-|x|}+8 a^{2}\left|w_{a}\right|^{4}\left|\partial_{i} w_{a}\right|^{2}+\frac{1}{4}\left|\partial_{i} w_{a}\right|^{2}, \\
\left|\varepsilon_{a}^{2} \Omega\left(i \partial_{j} w_{a}, \partial_{i} w_{a}\right)\right| \leq \frac{\varepsilon_{a}^{2} \Omega}{2}\left[\left(\partial_{i} w_{a}\right)^{2}+\left(\partial_{j} w_{a}\right)^{2}\right] .
\end{gathered}
$$


It then follows from (4.12) that as $a \nearrow a^{*}$,

$$
\left(-\frac{1}{2} \Delta-a\left|w_{a}\right|^{2}-8 a^{2}\left|w_{a}\right|^{4}\right)\left|\nabla w_{a}\right|^{2} \leq C \varepsilon_{a}^{4} e^{-|x|} \text { in } \mathbb{R}^{2},
$$

where the constant $C>0$ is independent of $0<a<a^{*}$. Applying De Giorgi-Nash-Moser theory [33, Theorem 4.1], we obtain from (4.13) that for any $y \in \mathbb{R}^{2}$,

$$
\sup _{x \in B_{\frac{1}{2}}(y)}\left|\nabla w_{a}(x)\right|^{2} \leq C\left(\left\|\nabla w_{a}\right\|_{L^{2}\left(B_{1}(y)\right)}^{2}+\varepsilon_{a}^{4}\left\|e^{-|x|}\right\|_{L^{2}\left(B_{1}(y)\right)}\right)
$$

holds as $a \nearrow a^{*}$, where the constant $C>0$ is independent of $0<a<a^{*}$. Since Theorem 1.2 gives that $w_{a} \rightarrow \frac{w}{\sqrt{a^{*}}}$ strongly in $H^{1}\left(\mathbb{R}^{2}, \mathbb{C}\right)$ as $a \nearrow a^{*}$, the claim $(4.9)$ is now proved in view of (4.14).

We next prove the estimate (4.7). Since $\left(R_{a}, I_{a}\right)$ solves the system (4.6), $\left(R_{a}, I_{a}\right)$ satisfies the system (A.3) with

$$
\begin{gathered}
V_{1}(x)=V_{2}(x)=\frac{\varepsilon_{a}^{4} \Omega^{2}}{4}|x|^{2}+\varepsilon_{a}^{2} V_{\Omega}\left(\varepsilon_{a} x+x_{a}\right)-\varepsilon_{a}^{2} \mu_{a}, \delta=\frac{1}{18}, \\
b_{1}=-b_{2}=\varepsilon_{a}^{2} \Omega, \quad f_{1}(x)=a\left|w_{a}\right|^{2} R_{a}, \quad f_{2}(x)=a\left|w_{a}\right|^{2} I_{a} .
\end{gathered}
$$

We derive from (3.31) that as $a \nearrow a^{*}$,

$$
\left|f_{1}(x)\right|, \quad\left|f_{2}(x)\right| \leq C_{1} e^{-\frac{2|x|}{3}} \text { in } \mathbb{R}^{2},
$$

where $C_{1}>0$ is independent of $0<a<a^{*}$. Together with (4.9), the above estimate of $f_{1}(x)$ and $f_{2}(x)$ then yields that as $a \nearrow a^{*}$, the assumption (A.1) of Lemma A.1 is satisfied for some $P>0$ and $Q=\frac{2}{3}$. Since $V_{\Omega}(x)$ is a homogeneous function of degree 2 , we have $2 V_{\Omega}(x)=x \cdot \nabla V_{\Omega}(x)$, which implies that

$$
\left|\nabla V_{\Omega}(x)\right| \geq \frac{2 V_{\Omega}(x)}{|x|} \geq 2|x| \min _{x \in \mathbb{R}^{2}} V_{\Omega}\left(\frac{x}{|x|}\right):=C_{1}|x| .
$$

On the other hand, we also have

$$
\nabla V_{\Omega}(x)=\nabla\left[|x|^{2} V_{\Omega}\left(\frac{x}{|x|}\right)\right]=2 V_{\Omega}\left(\frac{x}{|x|}\right) x+\left.\frac{\nabla V_{\Omega}(y) \cdot x^{\perp}}{|x|} x^{\perp}\right|_{y=\frac{x}{|x|}},
$$

which implies that

$$
\left|\nabla V_{\Omega}(x)\right| \leq C_{2}|x|, \quad C_{2}:=3\left\|V_{\Omega}(x)\right\|_{C^{1}\left(\partial B_{1}\right)}>0 .
$$

We thus obtain from above that there exist positive constants $C_{1}>0$ and $C_{2}>0$ such that

$$
C_{1}|x| \leq\left|\nabla V_{\Omega}(x)\right| \leq C_{2}|x| \text { in } \mathbb{R}^{2} .
$$

Applying (4.15), one can check that as $a \nearrow a^{*}, V_{1}(x)$ and $V_{2}(x)$ satisfy the assumption (A.2) of Lemma A.1 for $\delta=\frac{1}{18}$. Therefore, the estimate (4.7) is now proved by applying Lemma A.1.

2. Denoting $\hat{u}_{a}(x):=\varepsilon_{a} u_{a}\left(\varepsilon_{a} x+x_{a}\right)$, we have $\left\|\nabla \hat{u}_{a}\right\|_{2}^{2}=1$ and

$$
\begin{aligned}
\int_{\mathbb{R}^{2}}\left|\nabla w_{a}(x)\right|^{2} d x & =\int_{\mathbb{R}^{2}}\left|\nabla\left(\hat{u}_{a}(x) e^{-i\left(\frac{\Omega \varepsilon_{a} x \cdot x_{a}^{\perp}}{2}-\theta_{a}\right)}\right)\right|^{2} d x \\
& =\int_{\mathbb{R}^{2}}\left|\nabla \hat{u}_{a}(x) e^{-i\left(\frac{\Omega \varepsilon_{a} x \cdot x_{a}^{\perp}}{2}-\theta_{a}\right)}-\frac{i \Omega \varepsilon_{a} x_{a}^{\perp}}{2} \hat{u}_{a}(x) e^{-i\left(\frac{\Omega \varepsilon_{a} x \cdot x_{a}^{\perp}}{2}-\theta_{a}\right)}\right|^{2} d x \\
& =\int_{\mathbb{R}^{2}}\left|\nabla \hat{u}_{a}(x)-\frac{i \Omega \varepsilon_{a} x_{a}^{\perp}}{2} \hat{u}_{a}(x)\right|^{2} d x \\
& =\int_{\mathbb{R}^{2}}\left\{\left|\nabla \hat{u}_{a}\right|^{2}+\frac{\varepsilon_{a}^{2} \Omega^{2}\left|x_{a}\right|^{2}}{4}\left|\hat{u}_{a}\right|^{2}-\Omega \varepsilon_{a} x_{a}^{\perp} \cdot\left(i \hat{u}_{a}, \nabla \hat{u}_{a}\right)\right\} d x \\
& =\int_{\mathbb{R}^{2}}\left|\nabla \hat{u}_{a}\right|^{2} d x-I I I=1-I I I .
\end{aligned}
$$


We obtain from (3.48) and (3.56) that as $a \nearrow a^{*}$,

$$
\begin{aligned}
|I I I|: & =\left|\int_{\mathbb{R}^{2}}\left\{\frac{\varepsilon_{a}^{2} \Omega^{2}\left|x_{a}\right|^{2}}{4}\left|\hat{u}_{a}\right|^{2}-\Omega \varepsilon_{a} x_{a}^{\perp} \cdot\left(i \hat{u}_{a}, \nabla \hat{u}_{a}\right)\right\} d x\right| \\
& \leq C \varepsilon_{a}^{4}+\varepsilon_{a}\left|\int_{\mathbb{R}^{2}} \Omega x_{a}^{\perp} \cdot\left(i \hat{u}_{a}, \nabla \hat{u}_{a}\right) d x\right| \\
& \leq C \varepsilon_{a}^{4}+\varepsilon_{a}\left|\int_{\mathbb{R}^{2}} \Omega x_{a}^{\perp} \cdot\left(i w_{a}, \nabla w_{a}\right) d x\right|+\frac{\Omega^{2}\left|x_{a}\right|^{2} \varepsilon_{a}^{2}}{2} \int_{\mathbb{R}^{2}}\left|w_{a}\right|^{2} d x \leq C \varepsilon_{a}^{4} .
\end{aligned}
$$

On the other hand, we infer from (3.5) and (3.7) that

$$
\varepsilon_{a}^{2} \mu_{a}=\varepsilon_{a}^{2} e_{F}(a)-\frac{a}{2} \varepsilon_{a}^{2} \int_{\mathbb{R}^{2}}\left|u_{a}\right|^{4} d x=\varepsilon_{a}^{2} e_{F}(a)-\frac{a}{2} \int_{\mathbb{R}^{2}}\left|w_{a}\right|^{4} d x .
$$

We thus derive from (4.16)-(4.18) and (3.51) that as $a \nearrow a^{*}$,

$$
\begin{aligned}
\left|1+\varepsilon_{a}^{2} \mu_{a}\right| & =\left.\left|\int_{\mathbb{R}^{2}}\right| \nabla w_{a}\right|^{2}+I I I+\varepsilon_{a}^{2} e_{F}(a)-\frac{a}{2} \int_{\mathbb{R}^{2}}\left|w_{a}\right|^{4} d x \mid \\
& \leq\left.\left|\int_{\mathbb{R}^{2}}\right| \nabla w_{a}\right|^{2}-\frac{a^{*}}{2} \int_{\mathbb{R}^{2}}\left|w_{a}\right|^{4} d x+\frac{a^{*}-a}{2} \int_{\mathbb{R}^{2}}\left|w_{a}\right|^{4} d x \mid+C \varepsilon_{a}^{4} \leq C \varepsilon_{a}^{4},
\end{aligned}
$$

where the estimate (3.55) is also used in the last inequality. Therefore, the estimate (4.8) is proved. This completes the proof of Lemma 4.2.

The proof of Proposition 4.1 also needs the following iteration results.

Lemma 4.3. Under the assumptions of Proposition 4.1, for any $\alpha \geq 0$ suppose there exists a constant $C(\alpha)>0$, independent of $0<a<a^{*}$, such that as a $\nearrow a^{*}$,

$$
\left|x^{\perp} \cdot \nabla R_{a}(x)\right| \leq C(\alpha) \varepsilon_{a}^{\alpha} e^{-\frac{1}{4}|x|} \text { in } \mathbb{R}^{2} .
$$

Then as a $\nearrow a^{*}$,

$$
\left|I_{a}(x)\right|, \quad\left|\nabla I_{a}(x)\right| \leq C C(\alpha) \varepsilon_{a}^{2+\alpha} e^{-\frac{1}{8}|x|} \text { in } \mathbb{R}^{2},
$$

where the constant $C>0$ is independent of $0<a<a^{*}$ and $\alpha \geq 0$.

Proof. Recall from (4.2) and (4.6) that $I_{a}$ satisfies

$$
\mathcal{L}_{a} I_{a}(x)=-\varepsilon_{a}^{2} \Omega\left(x^{\perp} \cdot \nabla R_{a}\right) \quad \text { in } \mathbb{R}^{2}, \quad \int_{\mathbb{R}^{2}} I_{a}(x) w(x) d x=0 .
$$

Multiplying (4.22) by $I_{a}$ and integrating over $\mathbb{R}^{2}$, we obtain from (4.20) that

$$
\int_{\mathbb{R}^{2}}\left(\mathcal{L}_{a} I_{a}\right) I_{a} d x=-\varepsilon_{a}^{2} \Omega \int_{\mathbb{R}^{2}}\left(x^{\perp} \cdot \nabla R_{a}\right) I_{a} d x \leq C C(\alpha) \varepsilon_{a}^{2+\alpha}\left\|I_{a}\right\|_{L^{2}\left(\mathbb{R}^{2}\right)} .
$$

Following (3.45), we also get that

$$
\begin{aligned}
\int_{\mathbb{R}^{2}}\left(\mathcal{L}_{a} I_{a}\right) I_{a} d x & \geq \int_{\mathbb{R}^{2}}\left[\left(\mathcal{L} I_{a}\right) I_{a}-\left(1+\varepsilon_{a}^{2} \mu_{a}\right) I_{a}^{2}-\left(a\left|w_{a}\right|^{2}-w^{2}\right) I_{a}^{2}\right] d x \\
& =\int_{\mathbb{R}^{2}}\left(\mathcal{L} I_{a}\right) I_{a} d x+o(1) \int_{\mathbb{R}^{2}} I_{a}^{2} d x \geq \frac{\rho}{2}\left\|I_{a}\right\|_{H^{1}\left(\mathbb{R}^{2}\right)}^{2},
\end{aligned}
$$

where the constant $\rho>0$ given in (3.45) is independent of $0<a<a^{*}$. Thus, we infer from (4.23) and (4.24) that

$$
\left\|I_{a}\right\|_{L^{2}\left(\mathbb{R}^{2}\right)} \leq\left\|I_{a}\right\|_{H^{1}\left(\mathbb{R}^{2}\right)} \leq C C(\alpha) \varepsilon_{a}^{2+\alpha} .
$$


On the other hand, we derive from (4.22) that $\left|I_{a}\right|^{2}$ satisfies the following equation

$$
\begin{aligned}
& {\left[-\frac{1}{2} \Delta+\left(\frac{\varepsilon_{a}^{4} \Omega^{2}}{4}|x|^{2}+\varepsilon_{a}^{2} V_{\Omega}\left(\varepsilon_{a} x+x_{a}\right)-\mu_{a} \varepsilon_{a}^{2}-a\left|w_{a}\right|^{2}\right)\right]\left|I_{a}\right|^{2}+\left|\nabla I_{a}\right|^{2}} \\
& =-\varepsilon_{a}^{2} \Omega\left(x^{\perp} \cdot \nabla R_{a}\right) I_{a} \text { in } \mathbb{R}^{2},
\end{aligned}
$$

which implies that

$$
-\frac{1}{2} \Delta\left|I_{a}\right|^{2}-\mu_{a} \varepsilon_{a}^{2}\left|I_{a}\right|^{2}-a\left|w_{a}\right|^{2}\left|I_{a}\right|^{2} \leq-\varepsilon_{a}^{2} \Omega\left(x^{\perp} \cdot \nabla R_{a}\right) I_{a} \text { in } \mathbb{R}^{2} .
$$

Since $\mu_{a} \varepsilon_{a}^{2} \rightarrow-1$ as $a \nearrow a^{*}$, by De Giorgi-Nash-Moser theory [33, Theorem 4.1], we obtain from (4.26) that for any $y \in \mathbb{R}^{2}$,

$$
\sup _{x \in B_{\frac{1}{2}(y)}}\left|I_{a}(x)\right|^{2} \leq C\left(\left\|I_{a}\right\|_{L^{2}\left(B_{1}(y)\right)}^{2}+\left\|\varepsilon_{a}^{2} \Omega\left(x^{\perp} \cdot \nabla R_{a}\right) I_{a}\right\|_{L^{2}\left(B_{1}(y)\right)}\right) .
$$

We hence deduce from (3.31), (4.25) and (4.27) that

$$
\left\|I_{a}\right\|_{L^{\infty}\left(\mathbb{R}^{2}\right)} \leq C C(\alpha) \varepsilon_{a}^{2+\alpha}
$$

and

$$
\left.|a| w_{a}\right|^{2} I_{a}-\varepsilon_{a}^{2} \Omega\left(x^{\perp} \cdot \nabla R_{a}\right) \mid \leq C C(\alpha) \varepsilon_{a}^{2+\alpha} e^{-\frac{|x|}{4}} \text { in } \mathbb{R}^{2},
$$

where the assumption (4.20) is used. Applying the comparison principle to (4.6), we derive from (4.28) and (4.29) that

$$
\left|I_{a}(x)\right| \leq C C(\alpha) \varepsilon_{a}^{2+\alpha} e^{-\frac{|x|}{4}} \text { in } \mathbb{R}^{2} .
$$

Furthermore, applying gradient estimates (see (3.15) in [25]) to the equation (4.22), we conclude from above that

$$
\left|\nabla I_{a}(x)\right| \leq C C(\alpha) \varepsilon_{a}^{2+\alpha} e^{-\frac{1}{8}|x|} \text { in } \mathbb{R}^{2},
$$

which therefore completes the proof of Lemma 4.3.

Proof of Proposition 4.1. Note from (4.1) and (4.6) that $\left(\hat{R}_{a}, I_{a}\right)$ satisfies the following system

$$
\left\{\begin{aligned}
\left(\mathcal{L}_{a}-w^{2}-\sqrt{a^{*}} R_{a} w\right) \hat{R}_{a} & =F_{a}(x)+\varepsilon_{a}^{2} \Omega\left(x^{\perp} \cdot \nabla I_{a}\right) \text { in } \mathbb{R}^{2}, \\
\mathcal{L}_{a} I_{a} & =-\varepsilon_{a}^{2} \Omega\left(x^{\perp} \cdot \nabla \hat{R}_{a}\right) \text { in } \mathbb{R}^{2}
\end{aligned}\right.
$$

where $F_{a}(x)$ is defined by

$$
F_{a}(x):=-\left[\frac{\varepsilon_{a}^{4} \Omega^{2}}{4}|x|^{2}+\varepsilon_{a}^{2} V_{\Omega}\left(\varepsilon_{a} x+x_{a}\right)-\varepsilon_{a}^{2} \mu_{a}-1-a\left|I_{a}\right|^{2}+\left(a^{*}-a\right) R_{a}^{2}\right] \frac{w}{\sqrt{a^{*}}} .
$$

Next, we shall divide the proof into three steps.

Step 1. We first prove the following $C^{1}$ estimates of $\hat{R}_{a}$ and $I_{a}$ :

$$
\left\|\hat{R}_{a}\right\|_{C^{1}\left(\mathbb{R}^{2}\right)},\left\|I_{a}\right\|_{C^{1}\left(\mathbb{R}^{2}\right)} \leq C \varepsilon_{a}^{2} \text { as a } \nearrow a^{*},
$$

where $C>0$ is independent of $0<a<a^{*}$.

Recall from Lemmas 4.2 and 4.3 that as $a \nearrow a^{*}$,

$$
\left|I_{a}(x)\right|,\left|\nabla I_{a}(x)\right| \leq C \varepsilon_{a}^{2} e^{-\frac{1}{8}|x|} \text { in } \mathbb{R}^{2} .
$$

We claim that there exists $C>0$, independent of $0<a<a^{*}$, such that as $a \nearrow a^{*}$,

$$
\left\|\hat{R}_{a}\right\|_{L^{\infty}\left(\mathbb{R}^{2}\right)} \leq C \varepsilon_{a}^{2} .
$$


On the contrary, suppose that the above claim is false, i.e., $\lim _{a \nearrow a^{*}} \frac{\left\|\hat{R}_{a}\right\|_{L^{\infty}\left(\mathbb{R}^{2}\right)}}{\varepsilon_{a}^{2}}=\infty$. Set $\bar{R}_{a}=$ $\frac{\hat{R}_{a}}{\left\|\hat{R}_{a}\right\|_{L^{\infty}\left(\mathbb{R}^{2}\right)}}$, then we get from (4.31) that $\bar{R}_{a}$ satisfies

$$
\left(\mathcal{L}_{a}-w^{2}-\sqrt{a^{*}} R_{a} w\right) \bar{R}_{a}=\frac{F_{a}(x)+\varepsilon_{a}^{2} \Omega\left(x^{\perp} \cdot \nabla I_{a}\right)}{\left\|\hat{R}_{a}\right\|_{L^{\infty}\left(\mathbb{R}^{2}\right)}} \text { in } \mathbb{R}^{2} .
$$

The estimate (4.34) and Lemma 4.2 imply that the right hand side of (4.36) satisfies

$$
\left|\frac{F_{a}(x)+\varepsilon_{a}^{2} \Omega\left(x^{\perp} \cdot \nabla I_{a}\right)}{\left\|\hat{R}_{a}\right\|_{L^{\infty}\left(\mathbb{R}^{2}\right)}}\right| \leq e^{-\frac{1}{16}|x|} \quad \text { in } \mathbb{R}^{2} .
$$

By the comparison principle, we then obtain from (4.36) that

$$
\left|\bar{R}_{a}(x)\right| \leq e^{\frac{R}{16}-\frac{1}{16}|x|} \text { in } \mathbb{R}^{2} / B_{R} .
$$

Let $z_{a} \in \mathbb{R}^{2}$ be a global maximal point of $\bar{R}_{a}$ in $\mathbb{R}^{2}$. We then have $\left|z_{a}\right| \leq 2 R$ uniformly in $0<a<a^{*}$ for sufficiently large $R>0$. Applying the elliptic regularity theory, there exists a subsequence, still denoted by $\left\{\bar{R}_{a}\right\}$, such that $\bar{R}_{a}(x) \rightarrow R_{0}(x)$ in $C_{l o c}^{1}\left(\mathbb{R}^{2}\right)$ as $a \nearrow a^{*}$. Hence, $R_{0}$ satisfies

$$
-\Delta R_{0}+\left(1-3 w^{2}\right) R_{0}=0 \text { in } \mathbb{R}^{2},
$$

which implies that $R_{0}=\sum_{i=1}^{2} c_{i} \frac{\partial w}{\partial x_{i}}$. On the other hand, by (4.3) and (4.34), we have

$$
\left|\nabla R_{0}(0)\right|=\lim _{a \nearrow a^{*}}\left|\frac{-I_{a}(0) \nabla I_{a}(0)}{\left[\frac{w(0)}{\sqrt{a^{*}}}+\hat{R}_{a}(0)\right]\left\|\hat{R}_{a}\right\|_{L^{\infty}\left(\mathbb{R}^{2}\right)}}\right|=0 .
$$

Because $\nabla R_{0}(0)=0$, by the non-degeneracy of $w(x)$ at the origin, we get that $c_{1}=c_{2}=0$ and hence $R_{0} \equiv 0$, which however contradicts to the fact that $1=\bar{R}_{a}\left(z_{a}\right) \rightarrow R_{0}\left(z_{0}\right)=1$ as $a \nearrow a^{*}$ for some $z_{0} \in \mathbb{R}^{2}$. Thus, the claim (4.35) holds true.

Applying (4.8), (4.34) and (4.35), by the comparison principle we derive from (4.31) that $\hat{R}_{a}$ satisfies

$$
\left|\hat{R}_{a}(x)\right| \leq C \varepsilon_{a}^{2} e^{-\frac{1}{16}|x|} \text { in } \mathbb{R}^{2} .
$$

Furthermore, using the gradient estimates (see (3.15) in [25]) again, it follows from (4.31) and (4.37) that

$$
\left|\nabla \hat{R}_{a}\right| \leq C \varepsilon_{a}^{2} e^{-\frac{1}{32}|x|} \quad \text { in } \mathbb{R}^{2} .
$$

Combining (4.34), (4.35) and (4.38), we therefore conclude that (4.33) holds true.

Step 2. We next prove that as $a \nearrow a^{*}$,

$$
\left|\hat{R}_{a}(x)\right|,\left|\nabla \hat{R}_{a}(x)\right|,\left|I_{a}(x)\right|,\left|\nabla I_{a}(x)\right| \leq C \varepsilon_{a}^{2} e^{-\frac{1}{2}|x|} \text { in } \mathbb{R}^{2},
$$

where the constant $C>0$ is independent of $0<a<a^{*}$.

Indeed, note from the system (4.31) that $\left(\hat{R}_{a}, I_{a}\right)$ satisfies the system (A.3) with

$$
\begin{gathered}
V_{1}(x)=V_{2}(x)=\frac{\varepsilon_{a}^{4} \Omega^{2}}{4}|x|^{2}+\varepsilon_{a}^{2} V_{\Omega}\left(\varepsilon_{a} x+x_{a}\right)-\varepsilon_{a}^{2} \mu_{a}, \\
f_{1}(x)=\left(w^{2}+\sqrt{a^{*}} R_{a} w\right) \hat{R}_{a}+F_{a}(x)+a\left|w_{a}\right|^{2} \hat{R}_{a}, f_{2}(x)=a\left|w_{a}\right|^{2} I_{a}, \\
b_{1}=-b_{2}=\varepsilon_{a}^{2} \Omega, P=C \varepsilon_{a}^{2}, Q=\frac{1}{2}, \delta=\frac{1}{4},
\end{gathered}
$$

where $F_{a}(x)$ is defined by (4.32). By Step 1, one can check from (4.15), (4.34), (4.37) and (4.38) that the assumptions (A.1) and (A.2) of Lemma A.1 are satisfied as $a \nearrow a^{*}$. We therefore conclude from Lemma A.1 that the estimate (4.39) of $\left(\hat{R}_{a}, I_{a}\right)$ holds true as $a \nearrow a^{*}$. 
Step 3. Following Step 2, we deduce from Lemma 4.3 that as $a \nearrow a^{*}$,

$$
\left|I_{a}(x)\right|,\left|\nabla I_{a}(x)\right| \leq C \varepsilon_{a}^{4} e^{-\frac{1}{8}|x|} \text { in } \mathbb{R}^{2},
$$

where $C>0$ is independent of $0<a<a^{*}$. By the definition of $F_{a}(x)$ in (4.32), this further implies that as $a \nearrow a^{*}$,

$$
\left|F_{a}(x)\right| \leq C \varepsilon_{a}^{4} e^{-\frac{1}{16}|x|} \text { in } \mathbb{R}^{2}
$$

where (3.31) is also used. Similar to (4.37) and (4.38), we then obtain that as $a \nearrow a^{*}$,

$$
\left|\hat{R}_{a}(x)\right|,\left|\nabla \hat{R}_{a}(x)\right| \leq C \varepsilon_{a}^{4} e^{-\frac{1}{32}|x|} \text { in } \mathbb{R}^{2} .
$$

Similar to Step 2, we further conclude from (4.40), (4.41) and Lemma A.1 that as $a \nearrow a^{*}$,

$$
\left|\hat{R}_{a}(x)\right|,\left|\nabla \hat{R}_{a}(x)\right|,\left|I_{a}(x)\right|,\left|\nabla I_{a}(x)\right| \leq C \varepsilon_{a}^{4} e^{-\frac{1}{2}|x|} \text { in } \mathbb{R}^{2}
$$

where $C>0$ is independent of $0<a<a^{*}$. Therefore, we infer from (4.42) and Lemma 4.3 that as $a \nearrow a^{*}$,

$$
\left|I_{a}(x)\right|,\left|\nabla I_{a}(x)\right| \leq C \varepsilon_{a}^{6} e^{-\frac{1}{8}|x|} \text { in } \mathbb{R}^{2},
$$

where $C>0$ is also independent of $0<a<a^{*}$. This completes the proof of Proposition 4.1.

\section{Nonexistence of Vortices}

Following the previous section's notations and results, in this section we shall prove Theorem 1.3 on the nonexistence of vortices, for which we shall employ the so-called method of inductive symmetry explained in Subsection 1.1. To prove Theorem 1.3, we focus on the typical case where $V(x)=|x|^{2}$ and $0<\Omega<\Omega^{*}$ is fixed. In this case, one can check that $\Omega^{*}=2$ and $y_{0}=(0,0)$ is a unique and non-degenerate critical point of $H(y)$, see the assumption $\left(V_{1}\right)$ in Section 4 , and therefore Proposition 4.1 is applicable.

Under the above assumptions of Theorem 1.3, suppose $u_{a}$ is a complex-valued minimizer of $e_{F}(a)$ as $a \nearrow a^{*}$, and let $w_{a}$ be defined by (4.1) for the rescaling form of $u_{a}$ with a constant phase. Denote

$$
w_{a}(x):=R_{a}(x)+i I_{a}(x)=\frac{w(x)}{\sqrt{a^{*}}}+\hat{R}_{a}(x)+i I_{a}(x), \quad \varepsilon_{a}:=\left(\int_{\mathbb{R}^{2}}\left|\nabla u_{a}\right|^{2} d x\right)^{-\frac{1}{2}}>0,
$$

where $R_{a}(x), \hat{R}_{a}(x)$ and $I_{a}(x)$ are real-valued functions in $H^{1}\left(\mathbb{R}^{2}\right)$. Proposition 4.1 gives us the first estimates of $\hat{R}_{a}(x)$ and $I_{a}(x)$ as $a \nearrow a^{*}$. In this section, we first apply Proposition 4.1 to deriving Proposition 5.1 on the first estimate of the difference $R_{a}-v_{a}$ as $a \nearrow a^{*}$, instead of the difference $R_{a}-\frac{w}{\sqrt{a^{*}}}$, where $v_{a}>0$ defined in (5.5) denotes the rescaled real-valued minimizer of $e_{F}(a)$ at zero rotation $\Omega=0$. Note from [29] that $v_{a}>0$ is radially symmetric and unique as a $\nearrow a^{*}$. We then analyze the linearized operator $\mathcal{N}_{a}$ in Subsection 5.1, see (5.16) for the definition of $\mathcal{N}_{a}$. To finish the proof of Theorem 1.3, in Subsection 5.2 we shall apply the induction process to showing that the non-radial part of $R_{a}-v_{a}$ is arbitrarily small and

$$
\text { both } I_{a}(x) \equiv 0 \text { and } x_{a} \equiv 0 \text { as } a \nearrow a^{*} \text {, }
$$

where $x_{a} \in \mathbb{R}^{2}$ denotes the unique global maximal point of $\left|u_{a}\right|$ as $a \nearrow a^{*}$. This is the most crucial part in proving Theorem 1.3.

In order to establish Theorem 1.3 , we now denote $\hat{v}_{a}>0$ to be a radially symmetric real-valued minimizer of the following functional

$$
e_{a}=\inf _{\left\{u \in \mathbb{H},\|u\|_{2}^{2}=1\right\}} E_{a}(u),
$$


where the space $\mathbb{H}$ is defined by

$$
\mathbb{H}:=\left\{u \in H^{1}\left(\mathbb{R}^{2}\right): \int_{\mathbb{R}^{2}}|x|^{2} u^{2} d x<\infty\right\},
$$

and $E_{a}(u)$ satisfies

$$
E_{a}(u)=\int_{\mathbb{R}^{2}}\left(|\nabla u|^{2}+|x|^{2} u^{2}\right)-\frac{a}{2} \int_{\mathbb{R}^{2}} u^{4} d x .
$$

Since $\hat{v}_{a}>0$ is a minimizer of $e_{a}$, it satisfies the following Euler-Lagrange equation

$$
-\Delta \hat{v}_{a}(x)+|x|^{2} \hat{v}_{a}(x)=\beta_{a} \hat{v}_{a}(x)+a \hat{v}_{a}^{3}(x) \text { in } \mathbb{R}^{2},
$$

where $\beta_{a} \in \mathbb{R}$ is a Lagrange multiplier. Note that $\beta_{a}$ satisfies

$$
\beta_{a}=e_{a}-\frac{a}{2} \int_{\mathbb{R}^{2}} \hat{v}_{a}^{4} d x<0 \text { as } a \nearrow a^{*},
$$

where $\hat{v}_{a}>0$ is a unique positive minimizer of $e_{a}$ as $a \nearrow a^{*}$ in view of [29, Theorem 1.3].

Denote

$$
v_{a}(x)=\varepsilon_{a} \hat{v}_{a}\left(\varepsilon_{a} x\right), \quad \varepsilon_{a}:=\left(\int_{\mathbb{R}^{2}}\left|\nabla u_{a}\right|^{2} d x\right)^{-\frac{1}{2}}>0 .
$$

It then follows from (5.3) that $v_{a}>0$ satisfies

$$
-\Delta v_{a}(x)+\varepsilon_{a}^{4}|x|^{2} v_{a}(x)=\varepsilon_{a}^{2} \beta_{a} v_{a}(x)+a v_{a}^{3}(x) \text { in } \mathbb{R}^{2} .
$$

Moreover, since $\varepsilon_{a}>0$ satisfies (5.5), we obtain from (3.54) that

$$
\varepsilon_{a}=\alpha_{a}+o\left(\alpha_{a}\right) \quad \text { as } a \nearrow a^{*}, \text { where } \quad \alpha_{a}:=\frac{\left(a^{*}-a\right)^{\frac{1}{4}}}{\lambda}>0 .
$$

Following Proposition 4.1 and Lemma A.2, we begin with the following estimates as $a \nearrow a^{*}$ :

Proposition 5.1. Under the assumptions of Theorem 1.3, let $\varepsilon_{a}>0$ and $\alpha_{a}>0$ be defined by (5.5) and (5.7), respectively. Then as a $\nearrow a^{*}$,

(i). $\left|x_{a}\right| \leq C \varepsilon_{a}^{5}$;

(ii). $\left|\varepsilon_{a}-\alpha_{a}\right| \leq C \alpha_{a}^{3}$;

(iii). $\varepsilon_{a}^{2}\left|\beta_{a}-\mu_{a}\right| \leq C \alpha_{a}^{2}$;

(iv). $\left|R_{a}-v_{a}\right|,\left|\nabla\left(R_{a}-v_{a}\right)\right| \leq C \alpha_{a}^{2} e^{-\frac{1}{2}|x|}$ in $\mathbb{R}^{2}$,

where $x_{a} \in \mathbb{R}^{2}$ denotes a global maximal point of $\left|u_{a}\right|, R_{a}=R_{a}(x)$ is defined by (4.1), and $\mu_{a} \in \mathbb{R}$ satisfies (3.5).

Proof. 1. Multiplying the first equation of (4.31) by $\partial_{i} w:=\frac{\partial w}{\partial x_{i}}$ and then integrating over $\mathbb{R}^{2}$, we obtain that for $i=1,2$,

$$
\int_{\mathbb{R}^{2}} \partial_{i} w\left(\mathcal{L}_{a}-w^{2}-\sqrt{a^{*}} R_{a} w\right) \hat{R}_{a}=\int_{\mathbb{R}^{2}}\left(g_{1 a}+g_{2 a}\right) \partial_{i} w
$$

where

$$
g_{1 a}(x)=-\left[\frac{\varepsilon_{a}^{4} \Omega^{2}}{4}|x|^{2}+\varepsilon_{a}^{4}\left(1-\frac{\Omega^{2}}{4}\right)\left|x+\varepsilon_{a}^{-1} x_{a}\right|^{2}-\varepsilon_{a}^{2} \mu_{a}-1+\left(a^{*}-a\right) R_{a}^{2}\right] \frac{w}{\sqrt{a^{*}}},
$$

and

$$
g_{2 a}(x)=\frac{a w\left|I_{a}\right|^{2}}{\sqrt{a^{*}}}+\varepsilon_{a}^{2} \Omega\left(x^{\perp} \cdot \nabla I_{a}\right) .
$$


Applying Proposition 4.1 gives that as $a \nearrow a^{*}$,

$$
\left|\int_{\mathbb{R}^{2}} g_{2 a}(x) \partial_{i} w(x) d x\right| \leq C \varepsilon_{a}^{8}
$$

where $C>0$ is independent of $0<a<a^{*}$.

As for $\int_{\mathbb{R}^{2}} g_{1 a} \partial_{i} w$, we notice that

$$
\begin{aligned}
\int_{\mathbb{R}^{2}} g_{1 a} \partial_{i} w= & -\int_{\mathbb{R}^{2}} \partial_{i} w\left[\frac{\varepsilon_{a}^{4} \Omega^{2}}{4}|x|^{2}+\varepsilon_{a}^{4}\left(1-\frac{\Omega^{2}}{4}\right)\left|x+\varepsilon_{a}^{-1} x_{a}\right|^{2}-\varepsilon_{a}^{2} \mu_{a}-1+\left(a^{*}-a\right) R_{a}^{2}\right] \frac{w}{\sqrt{a^{*}}} \\
= & -\left(1-\frac{\Omega^{2}}{4}\right) \varepsilon_{a}^{4} \int_{\mathbb{R}^{2}} \partial_{i} w\left[\left|x+\varepsilon_{a}^{-1} x_{a}\right|^{2}-|x|^{2}\right] \frac{w}{\sqrt{a^{*}}} \\
& -\int_{\mathbb{R}^{2}} \partial_{i} w\left[\left(a^{*}-a\right)\left(R_{a}+\frac{w}{\sqrt{a^{*}}}\right) \hat{R}_{a}\right] \frac{w}{\sqrt{a^{*}}}
\end{aligned}
$$

due to the radial symmetry of $w$. We then obtain from above that as $a \nearrow a^{*}$,

$$
\begin{aligned}
\int_{\mathbb{R}^{2}} g_{1 a} \partial_{i} w= & -2 \varepsilon_{a}^{3}\left(1-\frac{\Omega^{2}}{4}\right) \int_{\mathbb{R}^{2}}[1+o(1)] \frac{\partial_{i} w^{2}}{2 \sqrt{a^{*}}} x_{a} \cdot x \\
& -\int_{\mathbb{R}^{2}} \partial_{i} w\left[\left(a^{*}-a\right)\left(R_{a}+\frac{w}{\sqrt{a^{*}}}\right) \hat{R}_{a}\right] \frac{w}{\sqrt{a^{*}}} \\
:= & -2 \varepsilon_{a}^{3}\left(1-\frac{\Omega^{2}}{4}\right) \int_{\mathbb{R}^{2}}[1+o(1)] \frac{\partial_{i} w^{2}}{2 \sqrt{a^{*}}} x_{a} \cdot x-I .
\end{aligned}
$$

Note from Proposition 4.1 that as $a \nearrow a^{*}$,

$$
|I|:=\left|\int_{\mathbb{R}^{2}} \partial_{i} w\left[\left(a^{*}-a\right)\left(R_{a}+\frac{w}{\sqrt{a^{*}}}\right) \hat{R}_{a}\right] \frac{w}{\sqrt{a^{*}}}\right| \leq C \varepsilon_{a}^{8} .
$$

On the other hand, denoting the operator

$$
\hat{\mathcal{L}}:=-\Delta+1-3 w^{2} \quad \text { in } \mathbb{R}^{2},
$$

we have

$$
\begin{aligned}
& I I:= \int_{\mathbb{R}^{2}} \partial_{i} w\left(\mathcal{L}_{a}-w^{2}-\sqrt{a^{*}} R_{a} w\right) \hat{R}_{a} \\
&= \int_{\mathbb{R}^{2}} \partial_{i} w \hat{\mathcal{L}} \hat{R}_{a}+\int_{\mathbb{R}^{2}} \partial_{i} w\left(\mathcal{L}_{a}-w^{2}-\sqrt{a^{*}} R_{a} w-\hat{\mathcal{L}}\right) \hat{R}_{a} \\
&=\int_{\mathbb{R}^{2}} \partial_{i} w\left[\frac{\varepsilon_{a}^{4} \Omega^{2}}{4}|x|^{2}+\varepsilon_{a}^{4}\left(1-\frac{\Omega^{2}}{4}\right)\left|x+\varepsilon_{a}^{-1} x_{a}\right|^{2}-\varepsilon_{a}^{2} \mu_{a}-1+2 w^{2}\right. \\
&\left.\quad-a\left|w_{a}\right|^{2}-\sqrt{a^{*}} R_{a} w\right] \hat{R}_{a} .
\end{aligned}
$$

Hence, we obtain from above that as $a \nearrow a^{*}$,

$$
|I I|:=\left|\int_{\mathbb{R}^{2}} \partial_{i} w\left(\mathcal{L}_{a}-w^{2}-\sqrt{a^{*}} R_{a} w\right) \hat{R}_{a}\right| \leq C \varepsilon_{a}^{8},
$$

where (4.8) and Proposition 4.1 are used. Therefore, we derive from (5.8)-(5.11) that as $a \nearrow a^{*}$,

$$
\left|2 \varepsilon_{a}^{3} \int_{\mathbb{R}^{2}}[1+o(1)] \frac{\partial_{i} w^{2}}{2 \sqrt{a^{*}}} x_{a} \cdot x\right| \leq C \varepsilon_{a}^{8}, \quad i=1,2,
$$

which further implies that Proposition 5.1(i) holds true.

2. On the contrary, suppose Proposition 5.1(ii) is false. Then there exists a constant $C(a)>0$, where $C(a) \rightarrow \infty$ as $a \nearrow a^{*}$, such that

$$
\left|\varepsilon_{a}-\alpha_{a}\right| \geq C(a) \alpha_{a}^{3} \quad \text { as } a \nearrow a^{*} .
$$


Select the test function $w_{\tau}(x):=\frac{\tau w(\tau x)}{\|w\|_{2}}$, where $\tau=\lambda\left(a^{*}-a\right)^{-\frac{1}{4}}$ and $\lambda=\left(\int_{\mathbb{R}^{2}}|x|^{2} w^{2}(x) d x\right)^{\frac{1}{4}}$ is defined by (1.16). We then get that as $a \nearrow a^{*}$,

$$
e_{F}(a) \leq F_{a}\left(w_{\tau}\right) \leq \int_{\mathbb{R}^{2}}\left(\left|\nabla w_{\tau}\right|^{2}+|x|^{2}\left|w_{\tau}\right|^{2}\right) d x-\frac{a}{2} \int_{\mathbb{R}^{2}}\left|w_{\tau}\right|^{4} d x=\frac{2 \lambda^{2}\left(a^{*}-a\right)^{\frac{1}{2}}}{a^{*}} .
$$

On the other hand, we infer from Proposition 4.1 that

$$
\Omega \int_{\mathbb{R}^{2}} x^{\perp} \cdot\left(i w_{a}(x), \nabla w_{a}(x)\right) d x=\Omega \int_{\mathbb{R}^{2}} x^{\perp} \cdot\left(R_{a} \nabla I_{a}-I_{a} \nabla R_{a}\right) d x=O\left(\varepsilon_{a}^{10}\right) \text { as } a \nearrow a^{*} .
$$

Then from Proposition 5.1(i), (3.39) and Proposition 4.1 we derive that as $a \nearrow a^{*}$,

$$
\begin{aligned}
e_{F}(a) \geq & \frac{1}{\varepsilon_{a}^{2}} \int_{\mathbb{R}^{2}}\left|\nabla w_{a}(x)\right|^{2} d x-\frac{a}{2 \varepsilon_{a}^{2}} \int_{\mathbb{R}^{2}}\left|w_{a}(x)\right|^{4} d x \\
& +\varepsilon_{a}^{2} \int_{\mathbb{R}^{2}}\left(\frac{\Omega^{2}}{4}|x|^{2}+\frac{4-\Omega^{2}}{4}\left|x+\frac{x_{a}}{\varepsilon_{a}}\right|^{2}\right)\left|w_{a}(x)\right|^{2} d x-C \varepsilon_{a}^{10} \\
\geq & \frac{a^{*}-a}{2 \varepsilon_{a}^{2}} \int_{\mathbb{R}^{2}}\left|w_{a}(x)\right|^{4} d x+\varepsilon_{a}^{2} \int_{\mathbb{R}^{2}}|x|^{2}\left|w_{a}(x)\right|^{2} d x-C \varepsilon_{a}^{6} \\
\geq & \frac{a^{*}-a}{2 \varepsilon_{a}^{2}} \int_{\mathbb{R}^{2}}\left|\frac{w}{\sqrt{a^{*}}}\right|^{4} d x+\varepsilon_{a}^{2} \int_{\mathbb{R}^{2}}|x|^{2}\left|\frac{w}{\sqrt{a^{*}}}\right|^{2} d x-C \varepsilon_{a}^{6}:=\frac{1}{a^{*}} f\left(\varepsilon_{a}\right)-C \varepsilon_{a}^{6},
\end{aligned}
$$

where $f(t):=\left(a^{*}-a\right) t^{-2}+t^{2} \int_{\mathbb{R}^{2}}|x|^{2} w^{2}(x) d x$ for $t>0$. Since $f^{\prime}\left(\alpha_{a}\right)=0$, applying Taylor's expansion, we obtain from (5.14) that as $a \nearrow a^{*}$,

$$
\begin{aligned}
e_{F}(a) & \geq \frac{1}{a^{*}} f\left(\varepsilon_{a}\right)-C \varepsilon_{a}^{6}=\frac{1}{a^{*}}\left[f\left(\alpha_{a}\right)+8 \lambda^{4}\left(\varepsilon_{a}-\alpha_{a}\right)^{2}+O\left(\left|\varepsilon_{a}-\alpha_{a}\right|^{3}\right)\right]-C \varepsilon_{a}^{6} \\
& \geq \frac{2 \lambda^{2}\left(a^{*}-a\right)^{\frac{1}{2}}}{a^{*}}+\left(\frac{4 \lambda^{4} C^{2}(a)}{a^{*}}-C\right) \varepsilon_{a}^{6},
\end{aligned}
$$

where both (5.7) and (5.12) are used. Since $C(a) \rightarrow \infty$ as $a \nearrow a^{*}$, the above estimate however contradicts to (5.13). Therefore, Proposition 5.1(ii) is proved.

3. Following Proposition 5.1(ii) and Lemma A.2(i), one can deduce from (4.8) that as $a \nearrow a^{*}$,

$$
\left|\varepsilon_{a}^{2}\left(\beta_{a}-\mu_{a}\right)\right| \leq\left|1+\varepsilon_{a}^{2} \beta_{a}\right|+\left|1+\varepsilon_{a}^{2} \mu_{a}\right| \leq\left|1+\alpha_{a}^{2} \beta_{a}\right|+\left|\left(\alpha_{a}^{2}-\varepsilon_{a}^{2}\right) \beta_{a}\right|+C \alpha_{a}^{4} \leq C \alpha_{a}^{2},
$$

which then implies that Proposition 5.1(iii) holds true.

4. By Lemma A.2, we deduce from Proposition 4.1 that as $a \nearrow a^{*}$,

$$
\begin{aligned}
& \left|R_{a}(x)-v_{a}(x)\right| \\
\leq & \left|R_{a}(x)-\frac{w(x)}{\sqrt{a^{*}}}\right|+\left|\frac{w(x)}{\sqrt{a^{*}}}-\alpha_{a} \hat{v}_{a}\left(\alpha_{a} x\right)\right|+\left|\alpha_{a} \hat{v}_{a}\left(\alpha_{a} x\right)-v_{a}(x)\right| \\
\leq & C \varepsilon_{a}^{4} e^{-\frac{1}{2}|x|}+C \alpha_{a}^{4} e^{-\frac{2}{3}|x|}+C\left|\frac{\left(\alpha_{a}-\varepsilon_{a}\right)}{\alpha_{a}} \alpha_{a} \hat{v}_{a}\left(\alpha_{a} x\right)\right| \\
& +C\left|\frac{\left(\alpha_{a}-\varepsilon_{a}\right) x}{\alpha_{a}}\right|\left|\nabla\left(\alpha_{a} \hat{v}_{a}\left(\alpha_{a} x+\theta\left(\alpha_{a}-\varepsilon_{a}\right) x\right)\right)\right| \\
\leq & C \varepsilon_{a}^{2} e^{-\frac{1}{2}|x|} \text { in } \mathbb{R}^{2},
\end{aligned}
$$

where $\theta=\left(\theta_{1}, \theta_{2}\right)$ is a vector with $|\theta|<1$. Similarly, one can obtain the similar estimate of $\nabla\left(R_{a}-v_{a}\right)$ as $a \nearrow a^{*}$. This completes the proof of Proposition 5.1(iv), and we are done.

\subsection{Analysis of the linearized operator $\mathcal{N}_{a}$}

In view of (5.6), this subsection is devoted to the analysis of the linearized operator $\mathcal{N}_{a}: D\left(\mathcal{N}_{a}\right) \subset$ $L^{2}\left(\mathbb{R}^{2}\right) \longmapsto L^{2}\left(\mathbb{R}^{2}\right)$ defined as

$$
\mathcal{N}_{a}:=-\Delta+\left(\varepsilon_{a}^{4}|x|^{2}-\varepsilon_{a}^{2} \beta_{a}-3 a v_{a}^{2}\right),
$$


where $v_{a}>0$ and $\varepsilon_{a}>0$ are defined in (5.5), and the domain $D\left(\mathcal{N}_{a}\right)$ satisfies

$$
D\left(\mathcal{N}_{a}\right)=H^{2}\left(\mathbb{R}^{2}\right) \cap\left\{u \in L^{2}\left(\mathbb{R}^{2}\right): \int_{\mathbb{R}^{2}}|x|^{4} u^{2} d x<\infty\right\} .
$$

It then follows from [12, Corollary 1.5] that $\mathcal{N}_{a}$ is non-degenerate and $\operatorname{ker}\left(\mathcal{N}_{a}\right)=\{0\}$. The main aim of this subsection is to establish the following estimate:

Lemma 5.2. Under the assumptions of Theorem 1.3, for any $\alpha \geq 0$ suppose $T_{\alpha, a} \in D\left(\mathcal{N}_{a}\right)$ is the unique solution of the following problem

$$
\begin{gathered}
\mathcal{N}_{a} T_{\alpha, a}=f_{\alpha, a}(x) \in L^{2}\left(\mathbb{R}^{2}\right), \\
\left|\nabla T_{\alpha, a}(0)\right| \leq C(\alpha) \varepsilon_{a}^{2 \alpha},\left|f_{\alpha, a}(x)\right| \leq C(\alpha) \varepsilon_{a}^{2 \alpha} e^{-\frac{1}{16}|x|} \text { in } \mathbb{R}^{2},
\end{gathered}
$$

where $C(\alpha)>0$ depends only on $\alpha \geq 0$. Then there exists a positive constant $C>0$, independent of $\alpha$ and $a$, such that as $a \nearrow a^{*}$,

$$
\left|T_{\alpha, a}(x)\right|,\left|\nabla T_{\alpha, a}(x)\right| \leq C C(\alpha) \varepsilon_{a}^{2 \alpha} e^{-\frac{1}{32}|x|} \text { in } \mathbb{R}^{2} .
$$

To prove Lemma 5.2, motivated by [49, Lemma 4.2] we first employ Proposition 5.1 to discuss the following analytical properties of $\mathcal{N}_{a}$.

Theorem 5.3. Under the assumptions of Theorem 1.3, we have

(i). $\mathcal{N}_{a}^{-1}: L^{2}\left(\mathbb{R}^{2}\right) \longmapsto L^{2}\left(\mathbb{R}^{2}\right)$ exists and is a continuous linear operator. Moreover, the following estimate holds true:

$$
\|\psi(x)\|_{H^{2}\left(\mathbb{R}^{2}\right)} \leq C\left\|\mathcal{N}_{a} \psi(x)\right\|_{L^{2}\left(\mathbb{R}^{2}\right)}, \quad \text { if } \psi \in\left\{\frac{\partial w}{\partial x_{1}}, \frac{\partial w}{\partial x_{2}}\right\}^{\perp},
$$

where $C>0$ is a constant independent of $0<a<a^{*}$.

(ii). If $\phi(x) \in L^{2}\left(\mathbb{R}^{2}\right)$ is radially symmetric, then $\psi(x)=\mathcal{N}_{a}^{-1} \phi(x) \in D\left(\mathcal{N}_{a}\right)$ is also radially symmetric, where the space $D\left(\mathcal{N}_{a}\right)$ is defined by (5.17).

Proof. (i). For any given $f(x) \in L^{2}\left(\mathbb{R}^{2}\right)$, consider the following equation

$$
-\Delta u+\left(\varepsilon_{a}^{4}|x|^{2}-\varepsilon_{a}^{2} \beta_{a}-3 a v_{a}^{2}\right) u=f \text { in } \mathbb{R}^{2} .
$$

By [50, Theorem 4.1] or [52, Theorem XIII.67], the operator $\left(-\Delta+\left(\varepsilon_{a}^{4}|x|^{2}-\varepsilon_{a}^{2} \beta_{a}\right)\right)^{-1}$ is compact from $L^{2}\left(\mathbb{R}^{2}\right)$ onto $L^{2}\left(\mathbb{R}^{2}\right)$. Hence, the equation (5.21) is solvable, if and only if the following holds

$$
u-3 a\left(-\Delta+\left(\varepsilon_{a}^{4}|x|^{2}-\varepsilon_{a}^{2} \beta_{a}\right)\right)^{-1}\left(v_{a}^{2} u\right)=\left(-\Delta+\left(\varepsilon_{a}^{4}|x|^{2}-\varepsilon_{a}^{2} \beta_{a}\right)\right)^{-1} f .
$$

One can obtain from Proposition 5.1(iv) that $v_{a} \in L^{\infty}\left(\mathbb{R}^{2}\right)$, which further implies that $(-\Delta+$ $\left.\left(\varepsilon_{a}^{4}|x|^{2}-\varepsilon_{a}^{2} \beta_{a}\right)\right)^{-1} \circ v_{a}^{2}$ is a compact operator. By Riesz-Fredholm theory of compact linear operators, we thus deduce that the equation (5.22) is solvable, if and only if $\operatorname{ker} \mathcal{N}_{a}=\{0\}$, but the latter is true in view of $\left[12\right.$, Corally 1.5]. This gives that $\mathcal{N}_{a}^{-1}$ exists. Therefore, $\mathcal{N}_{a}$ is a one-to-one and continuous linear mapping from $D\left(\mathcal{N}_{a}\right)$ onto $L^{2}\left(\mathbb{R}^{2}\right)$, which implies that $\mathcal{N}_{a}^{-1}$ is also continuous by the open mapping theorem.

We next prove the estimate (5.20) as follows. By the above argument, for any given $\phi(x) \in$ $L^{2}\left(\mathbb{R}^{2}\right)$, assume $\psi \in D\left(\mathcal{N}_{a}\right)$ is the unique solution of the following equation

$$
\mathcal{N}_{a} \psi(x)=\phi(x) \text { in } \mathbb{R}^{2} .
$$

For simplicity, we denote

$$
d_{a}(x)=-\varepsilon_{a}^{2} \beta_{a}-1+3 w^{2}-3 a v_{a}^{2}, \quad \hat{\mathcal{L}}=-\Delta+1-3 w^{2} \text { in } \mathbb{R}^{2},
$$


so that

$$
\mathcal{N}_{a}=\hat{\mathcal{L}}+\varepsilon_{a}^{4}|x|^{2}+d_{a}(x) .
$$

By (1) of Lemma 3.2 and (iii)-(iv) of Proposition 5.1, we obtain that

$$
\left\|d_{a}\right\|_{C^{1}\left(\mathbb{R}^{2}\right)} \rightarrow 0 \text { as } a \nearrow a^{*} .
$$

Thus, we infer from (5.23) that

$$
\begin{aligned}
\int_{\mathbb{R}^{2}}|\phi(x)|^{2} d x & =\int_{\mathbb{R}^{2}}(\hat{\mathcal{L}} \psi)^{2} d x+\int_{\mathbb{R}^{2}}\left(\varepsilon_{a}^{4}|x|^{2} \psi+d_{a} \psi\right)^{2} d x+2 \int_{\mathbb{R}^{2}} \hat{\mathcal{L}} \psi\left(\varepsilon_{a}^{4}|x|^{2} \psi+d_{a} \psi\right) d x \\
& =I_{1}+I_{2}+I_{3} .
\end{aligned}
$$

Applying [49, Lemma 4.2], there exists a constant $\delta>0$ such that $I_{1}$ satisfies

$$
I_{1}:=\int_{\mathbb{R}^{2}}(\hat{\mathcal{L}} \psi)^{2} d x \geq \delta\|\psi\|_{H^{2}\left(\mathbb{R}^{2}\right)}^{2}, \quad \text { if } \psi \in\left\{\frac{\partial w}{\partial x_{1}}, \frac{\partial w}{\partial x_{2}}\right\}^{\perp} .
$$

We also derive that $I_{2}:=\int_{\mathbb{R}^{2}}\left(\varepsilon_{a}^{4}|x|^{2} \psi+d_{a} \psi\right)^{2} d x \geq 0$, and however $I_{3}$ satisfies

$$
\begin{aligned}
I_{3}:= & \int_{\mathbb{R}^{2}} 2 \hat{\mathcal{L}} \psi\left(\varepsilon_{a}^{4}|x|^{2} \psi+d_{a} \psi\right) d x \\
= & \int_{\mathbb{R}^{2}}\left\{2 \varepsilon_{a}^{4}\left(|x|^{2}|\nabla \psi|^{2}+2 \psi x \cdot \nabla \psi\right)+2 d_{a}|\nabla \psi|^{2}+2 \psi \nabla \psi \nabla d_{a}\right. \\
& \left.+2\left(1-3 w^{2}\right)\left(\varepsilon_{a}^{4}|x|^{2}+d_{a}\right) \psi^{2}\right\} d x \\
\geq & \int_{\mathbb{R}^{2}}\left[-2 \varepsilon_{a}^{4}|\psi|^{2}-2\left|d_{a}\right||\nabla \psi|^{2}-\left|\nabla d_{a}\right|\left(|\psi|^{2}+|\nabla \psi|^{2}\right)\right. \\
& \left.-2\left|d_{a}\right|\left|1-3 w^{2}\right| \psi^{2}-6 \varepsilon_{a}^{4} w^{2}|x|^{2} \psi^{2}\right] d x \\
\geq & -\int_{\mathbb{R}^{2}}\left[C \max \left\{\varepsilon_{a}^{4},\left|\nabla d_{a}\right|,\left|d_{a}\right|\right\} \psi^{2}+\left(2\left|d_{a}\right|+\left|\nabla d_{a}\right|\right)|\nabla \psi|^{2}\right] d x \\
\geq & -\frac{\delta}{2}\|\psi\|_{H^{2}\left(\mathbb{R}^{2}\right)}^{2} \text { as } a \nearrow a^{*} .
\end{aligned}
$$

By applying above estimates, we conclude from (5.24) that if $\psi \in\left\{\frac{\partial w}{\partial x_{1}}, \frac{\partial w}{\partial x_{2}}\right\}^{\perp}$, then as $a \nearrow a^{*}$,

$$
\int_{\mathbb{R}^{2}}|\phi(x)|^{2} d x=\int_{\mathbb{R}^{2}}\left(\hat{\mathcal{L}} \psi+\varepsilon_{a}^{4}|x|^{2} \psi+d_{a} \psi\right)^{2} d x \geq \frac{\delta}{2}\|\psi\|_{H^{2}\left(\mathbb{R}^{2}\right)}^{2},
$$

which thus implies that (5.20) holds.

(ii). Set $\bar{\psi}(x)=\frac{1}{2 \pi|x|} \int_{|y|=|x|} \psi(y) d y$ for $x \neq(0,0)$, and $\bar{\psi}(x)=\psi(0)$ for $x=(0,0)$, where $\psi$ satisfies (5.23). We then obtain from (5.23) that $\bar{\psi}(x) \in D\left(\mathcal{N}_{a}\right)$ satisfies $\mathcal{N}_{a} \bar{\psi}(x)=\overline{\mathcal{N}_{a} \psi(x)}=$ $\bar{\phi}(x)=\phi(x)=\mathcal{N}_{a} \psi(x)$ in $\mathbb{R}^{2}$. By the non-degeneracy of $\mathcal{N}_{a}$ (cf. [12, Corollary 1.5]), it further implies that $\psi(x)$ is also radially symmetric. This completes the proof of Theorem 5.3.

Proof of Lemma 5.2. Choose suitable constants $a_{\alpha, a}$ and $b_{\alpha, a}$ such that

$$
T_{\alpha, a}=a_{\alpha, a} \partial_{1} w+b_{\alpha, a} \partial_{2} w+\hat{T}_{\alpha, a}, \quad \int_{\mathbb{R}^{2}} \hat{T}_{\alpha, a} \partial_{i} w=0 \text { for } i=1,2,
$$

where and below we denote $\partial_{i} w=\frac{\partial w}{\partial x_{i}}$ for $i=1$ and 2 . Without loss of generality, we may assume $\left|a_{\alpha, a}\right| \geq\left|b_{\alpha, a}\right|$.

We first claim that as $a \nearrow a^{*}$,

$$
\left|b_{\alpha, a}\right| \leq\left|a_{\alpha, a}\right| \leq C C(\alpha) \varepsilon_{a}^{2 \alpha} .
$$


Indeed, following (5.25), we have

$$
\begin{aligned}
\mathcal{N}_{a} \hat{T}_{\alpha, a} & =f_{\alpha, a}-\mathcal{N}_{a}\left(a_{\alpha, a} \partial_{1} w+b_{\alpha, a} \partial_{2} w\right) \\
& =f_{\alpha, a}+\left(-\varepsilon_{a}^{4}|x|^{2}+\varepsilon_{a}^{2} \beta_{a}+1-3 w^{2}+3 a v_{a}^{2}\right)\left(a_{\alpha, a} \partial_{1} w+b_{\alpha, a} \partial_{2} w\right) \text { in } \mathbb{R}^{2} .
\end{aligned}
$$

By (5.20) and (5.19), we infer from the above equation that as $a \nearrow a^{*}$,

$$
\begin{aligned}
& \left\|\hat{T}_{\alpha, a}\right\|_{L^{\infty}\left(\mathbb{R}^{2}\right)} \leq C\left\|\hat{T}_{\alpha, a}\right\|_{H^{2}\left(\mathbb{R}^{2}\right)} \\
\leq & C\left\|f_{\alpha, a}+\left(-\varepsilon_{a}^{4}|x|^{2}+\varepsilon_{a}^{2} \beta_{a}+1-3 w^{2}+3 a v_{a}^{2}\right)\left(a_{\alpha, a} \partial_{1} w+b_{\alpha, a} \partial_{2} w\right)\right\|_{L^{2}\left(\mathbb{R}^{2}\right)} \\
\leq & C\left\|f_{\alpha, a}\right\|_{L^{2}\left(\mathbb{R}^{2}\right)}+C \varepsilon_{a}^{2}\left(\left|a_{\alpha, a}\right|+\left|b_{\alpha, a}\right|\right) \\
\leq & C C(\alpha) \varepsilon_{a}^{2 \alpha}+C \varepsilon_{a}^{2}\left|a_{\alpha, a}\right|,
\end{aligned}
$$

where Proposition 5.1 and Lemma A.2 are also used in the second inequality. By the gradient estimates, we then obtain from (5.19) and (5.27) that as $a \nearrow a^{*}$,

$$
\begin{aligned}
& \left|\nabla \hat{T}_{\alpha, a}(0)\right| \\
\leq & C\left\|f_{\alpha, a}+\left(-\varepsilon_{a}^{4}|x|^{2}+\varepsilon_{a}^{2} \beta_{a}+1-3 w^{2}+3 a v_{a}^{2}\right)\left(a_{\alpha, a} \partial_{1} w+b_{\alpha, a} \partial_{2} w\right)\right\|_{L^{\infty}\left(B_{1}\right)} \\
& +C\left\|\hat{T}_{\alpha, a}\right\|_{L^{\infty}\left(\partial B_{1}\right)} \\
\leq & C C(\alpha) \varepsilon_{a}^{2 \alpha}+C \varepsilon_{a}^{2}\left|a_{\alpha, a}\right| .
\end{aligned}
$$

On the other hand, we have for $\lambda_{\alpha, a}:=\frac{b_{\alpha, a}}{a_{\alpha, a}}$,

$$
\begin{aligned}
& \left|\nabla\left(a_{\alpha, a} \partial_{1} w+b_{\alpha, a} \partial_{2} w\right)(0)\right| \\
= & \left|a_{\alpha, a}\right| \sqrt{\left[\partial_{1} \partial_{1} w(0)+\lambda_{\alpha, a} \partial_{1} \partial_{2} w(0)\right]^{2}+\left[\partial_{1} \partial_{2} w(0)+\lambda_{\alpha, a} \partial_{2} \partial_{2} w(0)\right]^{2}} \\
\geq & \left|a_{\alpha, a}\right| \frac{\left|\partial_{1} \partial_{1} w(0) \partial_{2} \partial_{2} w(0)-\left[\partial_{1} \partial_{2} w(0)\right]^{2}\right|}{\sqrt{\left[\partial_{1} \partial_{2} w(0)\right]^{2}+\left[\partial_{2} \partial_{2} w(0)\right]^{2}}}:=\eta\left|a_{\alpha, a}\right|,
\end{aligned}
$$

where the constant $\eta>0$ is independent of $\alpha \geq 0$ and $0<a<a^{*}$. Since $w(0)$ is non-degenerate, i.e.,

$$
\partial_{1} \partial_{1} w(0) \partial_{2} \partial_{2} w(0)-\left[\partial_{1} \partial_{2} w(0)\right]^{2} \neq 0,
$$

we have $\eta>0$. Therefore, we deduce from (5.28) and (5.29) that as $a \nearrow a^{*}$,

$$
\begin{aligned}
\left|\nabla T_{\alpha, a}(0)\right| & =\left|\nabla \hat{T}_{\alpha, a}(0)+\nabla\left(a_{\alpha, a} \partial_{1} w+b_{\alpha, a} \partial_{2} w\right)(0)\right| \\
& \geq\left|\nabla\left(a_{\alpha, a} \partial_{1} w+b_{\alpha, a} \partial_{2} w\right)(0)\right|-\left|\nabla \hat{T}_{\alpha, a}(0)\right| \\
& \geq \frac{\eta}{2}\left|a_{\alpha, a}\right|-C C(\alpha) \varepsilon_{a}^{2 \alpha} .
\end{aligned}
$$

Under the assumption (5.19), we therefore infer from the above inequality that (5.26) holds true.

Under the assumption (5.19), we now obtain from (5.25)-(5.27) that

$$
\left\|T_{\alpha, a}\right\|_{L^{\infty}\left(\mathbb{R}^{2}\right)} \leq C\left|a_{\alpha, a}\right|+\left\|\hat{T}_{\alpha, a}\right\|_{L^{\infty}\left(\mathbb{R}^{2}\right)} \leq C C(\alpha) \varepsilon_{a}^{2 \alpha} .
$$

Note from (5.18) and (5.19) that $T_{\alpha, a}$ satisfies

$$
\left|\mathcal{N}_{a} T_{\alpha, a}\right| \leq C C(\alpha) \varepsilon_{a}^{2 \alpha} e^{-\frac{1}{16}|x|} \text { in } \mathbb{R}^{2} / B_{R},
$$

where $R>0$ is large enough. Since $-\varepsilon_{a}^{2} \beta_{a} \rightarrow 1$ as $a \nearrow a^{*}$, by the comparison principle we deduce from (5.30) and Proposition 5.1(iv) that as $a \nearrow a^{*}$,

$$
\left|T_{\alpha, a}(x)\right| \leq C C(\alpha) \varepsilon_{a}^{2 \alpha} e^{-\frac{1}{16}|x|} \text { in } \mathbb{R}^{2} / B_{R} .
$$

By gradient estimates, together with (5.30), we further conclude from above that as $a \nearrow a^{*}$,

$$
\left|\nabla T_{\alpha, a}(x)\right| \leq C C(\alpha) \varepsilon_{a}^{2 \alpha} e^{-\frac{1}{32}|x|} \text { in } \mathbb{R}^{2},
$$

which completes the proof of Lemma 5.2. 


\subsection{Proof of Theorem $\mathbf{1 . 3}$}

The main purpose of this subsection is to complete the proof of Theorem 1.3. Before that, we need the following induction argument.

Lemma 5.4. Under the assumptions of Theorem 1.3, for any $m \geq 2$ suppose $R_{a}(x)-v_{a}(x)$ can be decomposed as

$$
\begin{gathered}
Q_{a}(x):=R_{a}(x)-v_{a}(x)=\psi_{m, a}(|x|)+T_{m, a}(x), \\
\left|T_{m, a}(x)\right|, \quad\left|\nabla T_{m, a}(x)\right| \leq A^{m} \varepsilon_{a}^{2 m} e^{-\frac{1}{2}|x|} \text { in } \mathbb{R}^{2} \text { as a } \nearrow a^{*},
\end{gathered}
$$

where $\psi_{m, a}(|x|)$ is radially symmetric and the constant $A>0$ is independent of $m$ and $0<a<a^{*}$. Then the decomposition of (5.31) and (5.32) holds for $m+1$.

Proof. Recall first that $R_{a}(x)$ and $v_{a}(x)$ are defined in (4.1) and (5.5), respectively. Under the assumptions of Theorem 1.3, suppose $R_{a}=v_{a}+\psi_{m, a}+T_{m, a}$ holds for some $m \geq 2$, where $v_{a}$ and $\psi_{m, a}(|x|)$ are radially symmetric, and $T_{m, a}$ satisfies (5.32). For convenience, we always use the symbol $C$ to denote some positive constant independent of $A>0, m \geq 2$ and $0<a<a^{*}$. We also consider sufficiently small $\varepsilon_{a}>0$, so that $0<A \varepsilon_{a}<1$ as $a>0$ is sufficiently close to $a^{*}$ from below.

For any $m \geq 2$, by Proposition 5.1(iv), we obtain from (5.31) and (5.32) that

$$
\begin{aligned}
\left|\psi_{m, a}(x)\right|,\left|\nabla \psi_{m, a}(x)\right| & \leq C A^{m} \varepsilon_{a}^{2 m} e^{-\frac{1}{2}|x|}+C \varepsilon_{a}^{2} e^{-\frac{1}{2}|x|} \\
& \leq C \varepsilon_{a}^{m} e^{-\frac{1}{2}|x|}+C \varepsilon_{a}^{2} e^{-\frac{1}{2}|x|} \\
& \leq C \varepsilon_{a}^{2} e^{-\frac{1}{2}|x|} \text { in } \mathbb{R}^{2} .
\end{aligned}
$$

Since $v_{a}(|x|)+\psi_{m, a}(|x|)$ is radially symmetric, we derive from (5.31) and (5.32) that for any $m \geq 2$,

$$
\left|x^{\perp} \cdot \nabla R_{a}\right|=\left|x^{\perp} \cdot \nabla T_{m, a}\right| \leq C A^{m} \varepsilon_{a}^{2 m} e^{-\frac{1}{4}|x|} \text { in } \mathbb{R}^{2} .
$$

We then obtain from Lemma 4.3 that

$$
\left|I_{a}(x)\right|,\left|\nabla I_{a}(x)\right| \leq C A^{m} \varepsilon_{a}^{2(m+1)} e^{-\frac{1}{8}|x|} \text { in } \mathbb{R}^{2} .
$$

Since $R_{a}=v_{a}+\psi_{m, a}+T_{m, a}$, we deduce from (4.6) and (5.16) that

$$
\begin{aligned}
\mathcal{N}_{a} Q_{a}:= & \frac{\left(4-\Omega^{2}\right) \varepsilon_{a}^{4}}{4}\left(|x|^{2}-\left|x+\varepsilon_{a}^{-1} x_{a}\right|^{2}\right) R_{a}-\varepsilon_{a}^{2}\left(\beta_{a}-\mu_{a}\right) R_{a} \\
& +a\left(R_{a}+2 v_{a}\right) Q_{a}^{2}+\varepsilon_{a}^{2} \Omega\left(x^{\perp} \cdot \nabla I_{a}\right)+a I_{a}^{2} R_{a} \\
= & \varepsilon_{a}^{2} \Omega\left(x^{\perp} \cdot \nabla I_{a}\right)+a I_{a}^{2} R_{a}+B_{1, a}(x)+B_{2, a}(x) \text { in } \mathbb{R}^{2},
\end{aligned}
$$

where the radially symmetric function $B_{1, a}(x)$ satisfies

$$
B_{1, a}(x)=-\varepsilon_{a}^{2}\left(\beta_{a}-\mu_{a}\right)\left(v_{a}+\psi_{m, a}\right)+a\left(\psi_{m, a}^{3}+3 v_{a} \psi_{m, a}^{2}\right),
$$

and the non-radially symmetric function $B_{2, a}(x)$ satisfies

$$
\begin{aligned}
B_{2, a}(x)= & \frac{\left(4-\Omega^{2}\right) \varepsilon_{a}^{4}}{4}\left(|x|^{2}-\left|x+\varepsilon_{a}^{-1} x_{a}\right|^{2}\right) R_{a}-\varepsilon_{a}^{2}\left(\beta_{a}-\mu_{a}\right) T_{m, a} \\
& +a\left(3 \psi_{m, a}^{2}+3 \psi_{m, a} T_{m, a}+T_{m, a}^{2}+6 v_{a} \psi_{m, a}+3 v_{a} T_{m, a}\right) T_{m, a} .
\end{aligned}
$$

Following Theorem 5.3 and (5.36), there exists a unique function $\psi_{m+1, a} \in C^{2}\left(\mathbb{R}^{2}\right) \cap L^{\infty}\left(\mathbb{R}^{2}\right)$ of

$$
\mathcal{N}_{a} \psi_{m+1, a}=B_{1, a}(x) \text { in } \mathbb{R}^{2},
$$

and moreover, $\psi_{m+1, a}$ is also radially symmetric. We now define

$$
T_{m+1, a}(x):=Q_{a}(x)-\psi_{m+1, a}(x),
$$


so that $T_{m+1, a}(x)$ satisfies

$$
\mathcal{N}_{a} T_{m+1, a}=\varepsilon_{a}^{2} \Omega\left(x^{\perp} \cdot \nabla I_{a}\right)+a I_{a}^{2} R_{a}+B_{2, a} \text { in } \mathbb{R}^{2},
$$

due to (5.35). Therefore, under the decomposition of (5.39), the rest is to prove that (5.32) holds for $m+1$. We shall prove it via the following three steps.

Step 1. Since $T_{m, a}$ satisfies (5.32), we claim that

$$
\left|x_{a}\right| \leq C A^{m} \varepsilon_{a}^{2 m-1} \text { as } a \nearrow a^{*},
$$

where $x_{a}$ is the unique global maximal point of $\left|u_{a}\right|$ as $a \nearrow a^{*}$.

Indeed, since $B_{1, a}(x)$ defined in (5.36) is radially symmetric, we deduce from (5.35) that

$$
\int_{\mathbb{R}^{2}} \partial_{i} w \mathcal{N}_{a} Q_{a}=\Omega \varepsilon_{a}^{2} \int_{\mathbb{R}^{2}} \partial_{i} w\left(x^{\perp} \cdot \nabla I_{a}\right)+a \int_{\mathbb{R}^{2}} \partial_{i} w I_{a}^{2} R_{a}+\int_{\mathbb{R}^{2}} \partial_{i} w B_{2, a} .
$$

Following (5.37) and (5.42) yields that

$$
\begin{gathered}
\int_{\mathbb{R}^{2}} \frac{\left(4-\Omega^{2}\right) \varepsilon_{a}^{4}}{4} \partial_{i} w\left(|x|^{2}-\left|x+\varepsilon_{a}^{-1} x_{a}\right|^{2}\right) R_{a} \\
=\int_{\mathbb{R}^{2}} \partial_{i} w \mathcal{N}_{a} Q_{a}-\Omega \varepsilon_{a}^{2} \int_{\mathbb{R}^{2}} \partial_{i} w\left(x^{\perp} \cdot \nabla I_{a}\right)-a \int_{\mathbb{R}^{2}} \partial_{i} w I_{a}^{2} R_{a} \\
-\int_{\mathbb{R}^{2}} \partial_{i} w\left[\varepsilon_{a}^{2}\left(\mu_{a}-\beta_{a}\right) T_{m, a}+a\left(3 \psi_{m, a}^{2}+3 \psi_{m, a} T_{m, a}\right.\right. \\
\left.\left.\quad+T_{m, a}^{2}+6 v_{a} \psi_{m, a}+3 v_{a} T_{m, a}\right) T_{m, a}\right] .
\end{gathered}
$$

We obtain from (5.39) that for $\hat{\mathcal{L}}=-\Delta+1-3 w^{2}$,

$$
\begin{aligned}
\int_{\mathbb{R}^{2}} \partial_{i} w \mathcal{N}_{a} Q_{a} & =\int_{\mathbb{R}^{2}} \partial_{i} w \hat{\mathcal{L}} Q_{a}+\int_{\mathbb{R}^{2}} \partial_{i} w\left(\mathcal{N}_{a}-\hat{\mathcal{L}}\right) Q_{a} \\
& =\int_{\mathbb{R}^{2}} \partial_{i} w\left[\varepsilon_{a}^{4}|x|^{2}-\varepsilon_{a}^{2} \beta_{a}-1+3 w^{2}-3 a v_{a}^{2}\right] Q_{a} \\
& =\int_{\mathbb{R}^{2}} \partial_{i} w\left[\varepsilon_{a}^{4}|x|^{2}-\varepsilon_{a}^{2} \beta_{a}-1+3 w^{2}-3 a v_{a}^{2}\right] T_{m, a} .
\end{aligned}
$$

By Proposition 5.1 and Lemma A.2, we get from (5.32) and (5.44) that as $a \nearrow a^{*}$,

$$
\left|\int_{\mathbb{R}^{2}} \partial_{i} w \mathcal{N}_{a} Q_{a}\right|=\left|\int_{\mathbb{R}^{2}} \partial_{i} w\left(\varepsilon_{a}^{4}|x|^{2}-\varepsilon_{a}^{2} \beta_{a}-1+3 w^{2}-3 a v_{a}^{2}\right) T_{m, a}\right| \leq C A^{m} \varepsilon_{a}^{2(m+1)} .
$$

Note from Proposition 5.1 and (5.32) that as $a \nearrow a^{*}$,

$$
\left|\varepsilon_{a}^{2}\left(\mu_{a}-\beta_{a}\right) \int_{\mathbb{R}^{2}} \partial_{i} w T_{m, a}\right| \leq C A^{m} \varepsilon_{a}^{2(m+1)} .
$$

Because $\varepsilon_{a}>0$ is small enough such that $0<A \varepsilon_{a}<1$, we also have as $a \nearrow a^{*}$,

$$
a\left|\int_{\mathbb{R}^{2}} \partial_{i} w I_{a}^{2} R_{a}\right| \leq C A^{m} \varepsilon_{a}^{2(m+1)},
$$

and

$$
a\left|\int_{\mathbb{R}^{2}} \partial_{i} w\left(3 \psi_{m, a}^{2}+3 \psi_{m, a} T_{m, a}+T_{m, a}^{2}+6 v_{a} \psi_{m, a}+3 v_{a} T_{m, a}\right) T_{m, a}\right| \leq C A^{m} \varepsilon_{a}^{2(m+1)},
$$

due to (5.33) and (5.34). Using above estimates, we then conclude from (5.34) and (5.43) that as $a \nearrow a^{*}$,

$$
\left|\int_{\mathbb{R}^{2}} \frac{\left(4-\Omega^{2}\right) \varepsilon_{a}^{4}}{4} \partial_{i} w\left(|x|^{2}-\left|x+\varepsilon_{a}^{-1} x_{a}\right|^{2}\right) R_{a}\right| \leq C A^{m} \varepsilon_{a}^{2(m+1)} .
$$


Since

$$
\begin{aligned}
& \int_{\mathbb{R}^{2}} \frac{\left(4-\Omega^{2}\right) \varepsilon_{a}^{4}}{4} \partial_{i} w\left(|x|^{2}-\left|x+\varepsilon_{a}^{-1} x_{a}\right|^{2}\right) R_{a} \\
& =-\int_{\mathbb{R}^{2}} \frac{\left(4-\Omega^{2}\right) \varepsilon_{a}^{3}}{2} \partial_{i} w\left(x_{a} \cdot x\right) R_{a}-\int_{\mathbb{R}^{2}} \frac{\left(4-\Omega^{2}\right) \varepsilon_{a}^{4}}{4} \partial_{i} w \varepsilon_{a}^{-2} x_{a}^{2} T_{m, a} \\
& =\int_{\mathbb{R}^{2}} \frac{\left(4-\Omega^{2}\right) \varepsilon_{a}^{3}}{4} w^{2} x_{a}^{(i)}+O\left(A^{m} \varepsilon_{a}^{2(m+1)}\right), \quad i=1,2,
\end{aligned}
$$

where $x_{a}=\left(x_{a}^{(1)}, x_{a}^{(2)}\right)$, we further deduce from (5.45) that the claim (5.41) holds true.

Step 2. We claim that as $a \nearrow a^{*}$,

$$
\left|T_{m+1, a}\right|,\left|\nabla T_{m+1, a}\right| \leq C A^{m} \varepsilon_{a}^{2(m+1)} e^{-\frac{1}{32}|x|} \text { in } \mathbb{R}^{2} .
$$

Actually, following (5.41) we obtain from (5.32), (3.31) and (5.37) that as $a \nearrow a^{*}$,

$$
\left|B_{2, a}(x)\right|,\left|\nabla B_{2, a}(x)\right| \leq C A^{m} \varepsilon_{a}^{2(m+1)} e^{-\frac{1}{2}|x|} \text { in } \mathbb{R}^{2} .
$$

Since

$$
T_{m+1, a}(x)=Q_{a}(x)-\psi_{m+1, a}(x)=R_{a}(x)-\left[v_{a}(x)+\psi_{m+1, a}(x)\right],
$$

where $v_{a}(x)+\psi_{m+1, a}(x)$ is radially symmetric, we derive from (5.34) that as $a \nearrow a^{*}$,

$$
\left|\nabla T_{m+1, a}(0)\right|=\left|\nabla R_{a}(0)\right|=\left|-\frac{I_{a}(0) \nabla I_{a}(0)}{R_{a}(0)}\right| \leq C A^{m} \varepsilon_{a}^{2(m+1)} .
$$

In view of (5.34), (5.47) and (5.48), applying Lemma 5.2 we therefore conclude from (5.40) that the claim (5.46) holds true.

Step 3. In this step, we prove that (5.32) holds for $m+1$. Recall from (4.6), (5.39) and (5.40) that $\left(T_{m+1, a}, I_{a}\right)$ satisfies the following system

$$
\left\{\begin{aligned}
\mathcal{N}_{a} T_{m+1, a} & =\varepsilon_{a}^{2} \Omega\left(x^{\perp} \cdot \nabla I_{a}\right)+a I_{a}^{2} R_{a}+B_{2, a}(x) \text { in } \mathbb{R}^{2}, \\
\mathcal{L}_{a} I_{a} & =-\varepsilon_{a}^{2} \Omega\left(x^{\perp} \cdot \nabla T_{m+1, a}\right) \text { in } \mathbb{R}^{2} .
\end{aligned}\right.
$$

Hence, we obtain from (5.49) that $\left(T_{m+1, a}, I_{a}\right)$ satisfies the system (A.3) with

$$
\begin{gathered}
b_{1}=-b_{2}=\varepsilon_{a}^{2} \Omega, P=C A^{m} \varepsilon_{a}^{2(m+1)}, Q=2 \delta=\frac{1}{2}, \\
f_{1}(x)=3 a v_{a}^{2} T_{m+1, a}+a I_{a}^{2} R_{a}+B_{2, a}(x), f_{2}(x)=a\left|w_{a}\right|^{2} I_{a}, \\
V_{1}(x)=\varepsilon_{a}^{4}|x|^{2}-\varepsilon_{a}^{2} \beta_{a}, V_{2}(x)=\frac{\varepsilon_{a}^{4} \Omega^{2}|x|^{2}}{4}+\varepsilon_{a}^{4}\left(1-\frac{\Omega^{2}}{4}\right)\left|x+x_{a} \varepsilon_{a}^{-1}\right|^{2}-\varepsilon_{a}^{2} \mu_{a} .
\end{gathered}
$$

By Steps 1 and 2, we obtain from (5.34) that $\left(T_{m+1, a}, I_{a}\right)$ satisfies (A.4), and $V_{1}(x)$ and $V_{2}(x)$ satisfy the assumption (A.2) of Lemma A.1. Moreover, we infer from (5.34), (5.47) and (5.46) that $f_{1}(x)$ and $f_{2}(x)$ satisfy the assumption (A.1) of Lemma A.1. Therefore, we deduce from Lemma A.1 that

$$
\left|I_{a}\right|,\left|\nabla I_{a}\right|,\left|T_{m+1, a}\right|,\left|\nabla T_{m+1, a}\right| \leq C A^{m} \varepsilon_{a}^{2(m+1)} e^{-\frac{1}{2}|x|} \text { in } \mathbb{R}^{2} .
$$

Because the positive constant $C$ in (5.50) is independent of $A>0, m \geq 2$ and $0<a<a^{*}$, one can choose a sufficiently large constant $A$ such that $A>C$. Therefore, we conclude from (5.50) that (5.32) holds for $m+1$, and the proof is complete.

Proof of Theorem 1.3. Set $T_{1, a}(x):=R_{a}(x)-v_{a}(x)$, where $R_{a}(x)$ and $v_{a}(x)$ are defined in (4.1) and (5.5), respectively. We then obtain from Proposition 5.1(iv) that

$$
\left|T_{1, a}(x)\right|,\left|\nabla T_{1, a}(x)\right| \leq C_{1} \varepsilon_{a}^{2} e^{-\frac{1}{2}|x|} \text { in } \mathbb{R}^{2},
$$


where $C_{1}>0$ is independent of $0<a<a^{*}$. Stimulated by (5.35), let $\psi_{2, a} \in C^{2}\left(\mathbb{R}^{2}\right) \cap L^{\infty}\left(\mathbb{R}^{2}\right)$ be the unique solution of the following equation

$$
\mathcal{N}_{a} \psi_{2, a}=-\varepsilon_{a}^{2}\left(\beta_{a}-\mu_{a}\right) v_{a} \text { in } \mathbb{R}^{2},
$$

and set $T_{2, a}:=R_{a}-v_{a}-\psi_{2, a}$. Theorem 5.3(ii) then gives that $\psi_{2, a}(|x|)$ is radially symmetric. Moreover, based on (5.51), the same argument of proving Lemma 5.4 gives that there exists a constant $C_{2}>0$, independent of $0<a<a^{*}$, so that

$$
\left|T_{2, a}(x)\right|,\left|\nabla T_{2, a}(x)\right| \leq C_{2} \varepsilon_{a}^{4} e^{-\frac{1}{2}|x|} \text { in } \mathbb{R}^{2} \text { as a } \nearrow a^{*} .
$$

Take $A>0$ large enough that $A^{2}>C_{2}$, from which we then have

$$
\left|T_{2, a}(x)\right|,\left|\nabla T_{2, a}(x)\right| \leq A^{2} \varepsilon_{a}^{4} e^{-\frac{1}{2}|x|} \text { in } \mathbb{R}^{2} \text { as a } \nearrow a^{*} .
$$

By Lemma 5.4, we thus deduce from (5.52) that for any $m \geq 2$,

$$
\left|T_{m, a}(x)\right|,\left|\nabla T_{m, a}(x)\right| \leq A^{m} \varepsilon_{a}^{2 m} e^{-\frac{1}{2}|x|} \text { in } \mathbb{R}^{2} \text { as a } \nearrow a^{*} .
$$

Recall from (5.31) that $R_{a}(x)=\left[v_{a}(x)+\psi_{m, a}(|x|)\right]+T_{m, a}(x)$, where $v_{a}(x)+\psi_{m, a}(|x|)$ is radially symmetric. Applying Lemma 4.3, we then derive from above that for any $m \geq 2$,

$$
\left|I_{a}(x)\right|,\left|\nabla I_{a}(x)\right| \leq C A^{m} \varepsilon_{a}^{2 m+2} e^{-\frac{1}{8}|x|} \text { in } \mathbb{R}^{2} \text { as a } \nearrow a^{*} .
$$

Moreover, the proof of Lemma 5.4, see (5.41), gives that for any $m \geq 2$,

$$
\left|x_{a}\right| \leq C A^{m} \varepsilon_{a}^{2 m-1} \quad \text { as } a \nearrow a^{*},
$$

where $x_{a}$ is the unique global maximal point of $\left|u_{a}\right|$ as $a \nearrow a^{*}$. Finally, let $a<a^{*}$ be sufficiently close to $a^{*}$ such that $0<A \varepsilon_{a}<1$. We then conclude from (5.53) and (5.54) that for any $m \geq 2$,

$$
\left\|I_{a}\right\|_{C^{1}\left(\mathbb{R}^{2}\right)} \leq C \varepsilon_{a}^{m+2} \text { in } \mathbb{R}^{2} \text { and }\left|x_{a}\right| \leq C \varepsilon_{a}^{m-1} \text { as } a \nearrow a^{*},
$$

which therefore implies that both $I_{a}(x) \equiv 0$ and $x_{a} \equiv 0$ hold as $a \nearrow a^{*}$.

Since $I_{a}(x) \equiv 0$ and $x_{a} \equiv 0$ as $a \nearrow a^{*}$, we obtain from (4.1) and (4.6) that $R_{a}:=$ $\varepsilon_{a} u_{a}\left(\varepsilon_{a} x\right) e^{i \Omega \theta_{a}}$ is a real-valued function and satisfies

$$
-\Delta R_{a}+\left(\varepsilon_{a}^{4}|x|^{2}-\varepsilon_{a}^{2} \mu_{a}-a R_{a}^{2}\right) R_{a}=0 \text { in } \mathbb{R}^{2} .
$$

This also gives the absence of vortices for $u_{a}$ as $a \nearrow a^{*}$. Following (5.55), the same argument of [29, Theorem 1.3] gives the uniqueness of $R_{a}$ as $a \nearrow a^{*}$, which then implies the uniqueness of $u_{a}$, up to a constant phase. This completes the proof of Theorem 1.3.

\section{A Appendix}

In this appendix, we shall establish Lemmas A.1 and A.2 which are used in the proof of Theorem 1.3 .

Lemma A.1. For $i=1,2$, suppose that $f_{i}(x) \in C^{1}\left(\mathbb{R}^{2}\right)$ satisfies

$$
\left|f_{i}(x)\right|,\left|\nabla f_{i}(x)\right| \leq P e^{-Q|x|} \text { in } \mathbb{R}^{2}
$$

for some constants $P>0$ and $Q>0$, and assume that there exists $b_{i} \in \mathbb{R}$ such that $V_{i}(x) \in$ $C^{1}\left(\mathbb{R}^{2}\right)$ satisfies

$$
V_{i}(x)-\left|\nabla V_{i}\right|^{2}-\frac{b_{i}^{2}|x|^{2}}{4}-\left(\left|b_{1}\right|+\left|b_{2}\right|\right) \geq 2 Q^{2}+\delta \text { in } \mathbb{R}^{2}
$$


for some constant $\delta>0$. Let $\left(w_{1}, w_{2}\right) \in C^{3}\left(\mathbb{R}^{2}\right) \times C^{3}\left(\mathbb{R}^{2}\right)$ be a real solution of

$$
\left\{\begin{array}{l}
-\Delta w_{1}+V_{1}(x) w_{1}=b_{1}\left(x^{\perp} \cdot \nabla w_{2}\right)+f_{1}(x) \text { in } \mathbb{R}^{2}, \\
-\Delta w_{2}+V_{2}(x) w_{2}=b_{2}\left(x^{\perp} \cdot \nabla w_{1}\right)+f_{2}(x) \text { in } \mathbb{R}^{2}
\end{array}\right.
$$

satisfying

$$
\left|w_{i}(x)\right|,\left|\nabla w_{i}(x)\right| \rightarrow 0 \text { as }|x| \rightarrow \infty, \quad i=1,2 .
$$

Then for $i=1,2, w_{i}(x)$ and $\nabla w_{i}(x)$ satisfy

$$
\left|w_{i}(x)\right|,\left|\nabla w_{i}(x)\right| \leq C(\delta) P e^{-Q|x|} \text { in } \mathbb{R}^{2},
$$

where the constant $C(\delta)>0$ depends only on $\delta>0$.

Proof. We first prove that (A.5) holds for $w_{i}(x)$ with $i=1$ and 2. Actually, we obtain from (A.3) that

$$
\left\{\begin{array}{l}
{\left[-\frac{1}{2} \Delta+V_{1}(x)\right] w_{1}^{2}+\left|\nabla w_{1}\right|^{2}=b_{1}\left(x^{\perp} \cdot \nabla w_{2}\right) w_{1}+f_{1} w_{1} \text { in } \mathbb{R}^{2},} \\
{\left[-\frac{1}{2} \Delta+V_{2}(x)\right] w_{2}^{2}+\left|\nabla w_{2}\right|^{2}=b_{2}\left(x^{\perp} \cdot \nabla w_{1}\right) w_{2}+f_{2} w_{2} \text { in } \mathbb{R}^{2} .}
\end{array}\right.
$$

For $i, j=1,2$, we get that

$$
b_{i}\left(x^{\perp} \cdot \nabla w_{j}\right) w_{i}(x) \leq \frac{b_{i}^{2}|x|^{2}\left|w_{i}\right|^{2}}{4}+\left|\nabla w_{j}\right|^{2}, \quad f_{i}(x) w_{i}(x) \leq \frac{\delta}{2} w_{i}^{2}+C_{1}(\delta) f_{i}^{2},
$$

where $\delta>0$ is as in (A.2). Applying (A.1) and (A.2), it then follows from (A.6) that

$$
\left(-\Delta+4 Q^{2}+\delta\right)\left(w_{1}^{2}+w_{2}^{2}\right) \leq C_{1}(\delta) P^{2} e^{-2 Q|x|} \text { in } \mathbb{R}^{2} .
$$

Applying the comparison principle to (A.7), we thus deduce from (A.4) that

$$
w_{1}^{2}+w_{2}^{2} \leq C_{2}(\delta) P^{2} e^{-2 Q|x|} \text { in } \mathbb{R}^{2},
$$

which implies that (A.5) holds for $w_{i}(x)$ with $i=1$ and 2.

We next prove that (A.5) holds for $\left|\nabla w_{i}\right|$ with $i=1$ and 2 . For $i, j=1,2$, denote $\partial_{i} w_{j}(x)=$ $\frac{\partial w_{j}(x)}{\partial x_{i}}$, and let $\delta_{i j}$ be the Kronecker function satisfying $\delta_{i j}=1$ for $i=j$ and $\delta_{i j}=0$ for $i \neq j$. We then derive from (A.3) that $\left(\partial_{i} w_{1}, \partial_{i} w_{2}\right)$ satisfies

$$
\left\{\begin{array}{l}
{\left[-\Delta+V_{1}(x)\right] \partial_{i} w_{1}+\partial_{i} V_{1}(x) w_{1}=b_{1}\left[\left(x^{\perp} \cdot \nabla \partial_{i} w_{2}\right)-\delta_{i, 2} \partial_{1} w_{2}+\delta_{i 1} \partial_{2} w_{2}\right]+\partial_{i} f_{1}(x),} \\
{\left[-\Delta+V_{2}(x)\right] \partial_{i} w_{2}+\partial_{i} V_{2}(x) w_{2}=b_{2}\left[\left(x^{\perp} \cdot \nabla \partial_{i} w_{1}\right)-\delta_{i, 2} \partial_{1} w_{1}+\delta_{i 1} \partial_{2} w_{1}\right]+\partial_{i} f_{2}(x)}
\end{array}\right.
$$

The above system further yields that

$$
\left\{\begin{array}{l}
{\left[-\frac{1}{2} \Delta+V_{1}(x)\right]\left|\nabla w_{1}\right|^{2}+\sum_{i=1}^{2}\left|\nabla \partial_{i} w_{1}\right|^{2}+\sum_{i=1}^{2} \partial_{i} V_{1}(x) w_{1} \partial_{i} w_{1}} \\
=b_{1}\left[\sum_{i=1}^{2}\left(x^{\perp} \cdot \nabla \partial_{i} w_{2}\right) \partial_{i} w_{1}+\partial_{2} w_{2} \partial_{1} w_{1}-\partial_{1} w_{2} \partial_{2} w_{1}\right]+\sum_{i=1}^{2} \partial_{i} f_{1}(x) \partial_{i} w_{1} \text { in } \mathbb{R}^{2} \\
{\left[-\frac{1}{2} \Delta+V_{2}(x)\right]\left|\nabla w_{2}\right|^{2}+\sum_{i=1}^{2}\left|\nabla \partial_{i} w_{2}\right|^{2}+\sum_{i=1}^{2} \partial_{i} V_{2}(x) w_{2} \partial_{i} w_{2}} \\
=b_{2}\left[\sum_{i=1}^{2}\left(x^{\perp} \cdot \nabla \partial_{i} w_{1}\right) \partial_{i} w_{2}+\partial_{2} w_{1} \partial_{1} w_{2}-\partial_{1} w_{1} \partial_{2} w_{2}\right]+\sum_{i=1}^{2} \partial_{i} f_{2}(x) \partial_{i} w_{2} \text { in } \mathbb{R}^{2}
\end{array}\right.
$$

Note that for $i, j, l=1,2$ with $j \neq l$,

$$
\partial_{i} V_{j}(x) w_{j} \partial_{i} w_{j} \leq\left|\partial_{i} V_{j}(x)\right|^{2}\left|\partial_{i} w_{j}\right|^{2}+\frac{1}{4} w_{j}^{2}, \quad \partial_{i} f_{j}(x) \partial_{i} w_{j} \leq \frac{\delta}{2}\left(\partial_{i} w_{j}\right)^{2}+C_{1}(\delta)\left|\partial_{i} f_{j}(x)\right|^{2},
$$




$$
b_{l}\left(x^{\perp} \cdot \nabla \partial_{i} w_{j}\right) \partial_{i} w_{l} \leq \frac{b_{l}^{2}|x|^{2}\left|\partial_{i} w_{l}\right|^{2}}{4}+\left|\nabla \partial_{i} w_{j}\right|^{2}, \quad b_{l} \partial_{1} w_{j} \partial_{2} w_{l} \leq \frac{1}{2}\left|b_{l}\right|\left(\left|\nabla w_{1}\right|^{2}+\left|\nabla w_{2}\right|^{2}\right),
$$

where $\delta>0$ is again as in (A.2). Following above estimates, we infer from (A.2) that

$$
\left(-\Delta+4 Q^{2}+\delta\right)\left(\left|\nabla w_{1}\right|^{2}+\left|\nabla w_{2}\right|^{2}\right) \leq\left(\left|w_{1}\right|^{2}+\left|w_{2}\right|^{2}\right)+2 C_{1}(\delta)\left(\left|\nabla f_{1}\right|^{2}+\left|\nabla f_{2}\right|^{2}\right) \text { in } \mathbb{R}^{2} .
$$

Applying (A.1) and (A.2), since (A.5) holds for $w_{i}(x)$ with $i=1$ and 2, we deduce from the above equation that

$$
\left[-\Delta+4 Q^{2}+\delta\right]\left(\left|\nabla w_{1}\right|^{2}+\left|\nabla w_{2}\right|^{2}\right) \leq C_{3}(\delta) P^{2} e^{-2 Q|x|} \text { in } \mathbb{R}^{2} .
$$

Applying the comparison principle to (A.10), we derive from (A.4) that

$$
\left|\nabla w_{1}\right|^{2}+\left|\nabla w_{2}\right|^{2} \leq C_{4}(\delta) P^{2} e^{-2 Q|x|} \text { in } \mathbb{R}^{2} .
$$

We therefore conclude from above that (A.5) holds for $\left|\nabla w_{i}\right|$ with $i=1$ and 2 , and we are done.

The rest part of this appendix is to derive some estimates used in the proof of Proposition 5.1 , for which we consider the following minimization problem

$$
e_{a}=\inf _{\left\{u \in \mathbb{H},\|u\|_{2}^{2}=1\right\}} E_{a}(u),
$$

where $\mathbb{H}:=\left\{u \in H^{1}\left(\mathbb{R}^{2}, \mathbb{R}\right): \int_{\mathbb{R}^{2}}|x|^{2} u^{2} d x<\infty\right\}$, and $E_{a}(u)$ is defined by

$$
E_{a}(u)=\int_{\mathbb{R}^{2}}\left(|\nabla u|^{2}+|x|^{2} u^{2}\right) d x-\frac{a}{2} \int_{\mathbb{R}^{2}} u^{4} d x, \quad a>0 .
$$

As stated in Theorem 2.1, $e_{a}$ admits minimizers if and only if $0<a<a^{*}=\|w\|_{2}^{2}$. Let $\hat{v}_{a}>0$ be a real minimizer of $e_{a}$ as $a \nearrow a^{*}$. Then $\hat{v}_{a}>0$ satisfies the following Euler-Lagrange equation

$$
-\Delta \hat{v}_{a}+|x|^{2} \hat{v}_{a}=\beta_{a} \hat{v}_{a}+a \hat{v}_{a}^{3} \text { in } \mathbb{R}^{2},
$$

where $\beta_{a} \in \mathbb{R}$ is the Lagrange multiplier and satisfies

$$
\beta_{a}=e_{a}-\frac{a}{2} \int_{\mathbb{R}^{2}} \hat{v}_{a}^{4}<0 \text { as } a \nearrow a^{*} .
$$

We also denote

$$
\alpha_{a}=\frac{\left(a^{*}-a\right)^{\frac{1}{4}}}{\lambda}>0 .
$$

The following lemma gives the estimates of $\beta_{a}$ and $\hat{v}_{a}$ as $a \nearrow a^{*}$ :

Lemma A.2. Let $\hat{v}_{a}>0$ be a real minimizer of $e_{a}$. Then as a $\nearrow a^{*}$,

(i). $1+\alpha_{a}^{2} \beta_{a}=O\left(\alpha_{a}^{4}\right)$;

(ii). $\left|\alpha_{a} \hat{v}_{a}\left(\alpha_{a} x\right)\right| \leq C e^{-\frac{3}{4}|x|},\left|\nabla\left(\alpha_{a} \hat{v}_{a}\left(\alpha_{a} x\right)\right)\right| \leq C e^{-\frac{2}{3}|x|}$ in $\mathbb{R}^{2}$;

(iii). $\max _{i, j=1,2}\left|\partial_{i} \partial_{j}\left(\alpha_{a} \hat{v}_{a}\left(\alpha_{a} x\right)\right)\right| \leq C e^{-\frac{7}{12}|x|}$ in $\mathbb{R}^{2}$;

(iv). $\left|\alpha_{a} \hat{v}_{a}\left(\alpha_{a} x\right)-\frac{w(x)}{\sqrt{a^{*}}}\right| \leq C \alpha_{a}^{4} e^{-\frac{2}{3}|x|},\left|\nabla\left(\alpha_{a} \hat{v}_{a}\left(\alpha_{a} x\right)-\frac{w(x)}{\sqrt{a^{*}}}\right)\right| \leq C \alpha_{a}^{4} e^{-\frac{1}{2}|x|}$ in $\mathbb{R}^{2} ;$

All above constants $C>0$ are independent of $0<a<a^{*}$. 
Proof. 1. The estimate (i) follows directly from (3.1) and (3.37) in [29].

2. Denote $\bar{v}_{a}(x)=\alpha_{a} \hat{v}\left(\alpha_{a} x\right)$. It then follows from (A.13) that $\bar{v}_{a}$ satisfies

$$
-\Delta \bar{v}_{a}+\alpha_{a}^{4}|x|^{2} \bar{v}_{a}=\alpha_{a}^{2} \beta_{a} \bar{v}_{a}+a \bar{v}_{a}^{3} \text { in } \mathbb{R}^{2} .
$$

Similar to (3.13) in [31], one can obtain from (A.14) that $\left\|\bar{v}_{a}\right\|_{L^{\infty}\left(\mathbb{R}^{2}\right)} \leq C$ and $\bar{v}_{a}(x) \rightarrow 0$ as $|x| \rightarrow \infty$ for all $0<a<a^{*}$. Since the estimate (i) gives that $\alpha_{a}^{2} \beta_{a} \rightarrow-1$ as $a \nearrow a^{*}$, we derive from (A.14) that as $a \nearrow a^{*}$,

$$
-\Delta \bar{v}_{a}+\frac{9}{16} \bar{v}_{a} \leq 0 \text { in } \mathbb{R}^{2} / B_{R},
$$

where $R>0$ is large enough. By the comparison principle, we then deduce from (A.15) that as a $\nearrow a^{*}$,

$$
\left|\bar{v}_{a}(x)\right| \leq C e^{-\frac{3}{4}|x|} \text { in } \mathbb{R}^{2} / B_{R},
$$

which implies that there exists a sufficiently large constant $C>0$ such that as $a \nearrow a^{*}$,

$$
\left|\bar{v}_{a}(x)\right| \leq C e^{-\frac{3}{4}|x|} \text { in } \mathbb{R}^{2} .
$$

Next, we give the estimate of $\nabla \bar{v}_{a}(x)$ as follows. Denoting $\bar{v}_{a, j}:=\frac{\partial \bar{v}_{a}}{\partial x_{j}}, j=1,2$, we then infer from (A.14) that $\bar{v}_{a, j}$ satisfies

$$
-\Delta \bar{v}_{a, j}+\left(\alpha_{a}^{4}|x|^{2}-\alpha_{a}^{2} \beta_{a}-3 a \bar{v}_{a}^{2}\right) \bar{v}_{a, j}=-2 \alpha_{a}^{4} x_{j} \bar{v}_{a} \quad \text { in } \mathbb{R}^{2} .
$$

Applying (i) and (A.16), we have for $a \nearrow a^{*}$,

$$
-\alpha_{a}^{2} \beta_{a} \rightarrow 1,\left|x_{j} \bar{v}_{a}\right| \leq C e^{-\frac{2}{3}|x|} \text { in } \mathbb{R}^{2} / B_{R},
$$

where $R>0$ is large enough. Therefore, by the comparison principle, we deduce from (A.17) that as $a \nearrow a^{*}$,

$$
\left|\bar{v}_{a, j}(x)\right| \leq C e^{-\frac{2}{3}|x|} \text { in } \mathbb{R}^{2} / B_{R} .
$$

On the other hand, similar to (3.13) in [31] again, one can get from (A.17) that $\bar{v}_{a, j}(x)$ is bounded uniformly in $\mathbb{R}^{2}$ for $k$, which further implies that as $a \nearrow a^{*}$,

$$
\left|\bar{v}_{a, j}(x)\right| \leq C e^{-\frac{2}{3}|x|} \text { in } \mathbb{R}^{2}, \quad j=1,2,
$$

from which we then obtain the desired estimate of $\nabla \bar{v}_{a}$ as $a \nearrow a^{*}$. This gives the estimate of (ii).

3. By the exponential estimate (A.18), applying gradient estimates $[25,(3.15)]$ to (A.17) gives that as $a \nearrow a^{*}$,

$$
\left|\nabla \bar{v}_{a, j}(x)\right| \leq C e^{-\frac{7}{12}|x|} \text { in } \mathbb{R}^{2},
$$

where $\bar{v}_{a, j}:=\frac{\partial \bar{v}_{a}}{\partial x_{j}}$ for $j=1,2$. Taking $j=1,2$, we then obtain that for any $i, j=1,2$,

$$
\left|\partial_{i} \partial_{j} \bar{v}_{a}(x)\right| \leq C e^{-\frac{7}{12}|x|} \text { in } \mathbb{R}^{2}
$$

as $a \nearrow a^{*}$, which therefore gives the estimate of (iii).

4. Denote $\tilde{v}_{a}(x):=\bar{v}_{a}(x)-\frac{w}{\sqrt{a^{*}}}=\alpha_{a} \hat{v}_{a}\left(\alpha_{a} x\right)-\frac{w}{\sqrt{a^{*}}}$. We then infer from [29, Theorem 1.4] that as $a \nearrow a^{*}$,

$$
\left\|\tilde{v}_{a}(x)\right\|_{L^{\infty}\left(\mathbb{R}^{2}\right)} \leq C \alpha_{a}^{4} .
$$

Moreover, because $\tilde{v}_{a}(x)$ satisfies the following equation

$$
\begin{aligned}
& -\Delta \tilde{v}_{a}-\alpha_{a}^{2} \beta_{a} \tilde{v}_{a}-a\left(\bar{v}_{a}^{2}+\frac{w \bar{v}_{a}}{\sqrt{a^{*}}}+\frac{w^{2}}{a^{*}}\right) \tilde{v}_{a} \\
= & -\alpha_{a}^{4}|x|^{2} \bar{v}_{a}+\left(1+\alpha_{a}^{2} \beta_{a}\right) \frac{w}{\sqrt{a^{*}}}+\frac{\left(a-a^{*}\right) w^{3}}{\left(a^{*}\right)^{\frac{3}{2}}} \text { in } \mathbb{R}^{2},
\end{aligned}
$$


by the comparison principle, we obtain from (A.19) and (i) that as $a \nearrow a^{*}$,

$$
\left|\tilde{v}_{a}(x)\right| \leq C \alpha_{a}^{4} e^{-\frac{2}{3}|x|} \text { in } \mathbb{R}^{2} / B_{R},
$$

where $R>0$ is large enough. This further implies that as $a \nearrow a^{*}$,

$$
\left|\tilde{v}_{a}(x)\right| \leq C \alpha_{a}^{4} e^{-\frac{2}{3}|x|} \text { in } \mathbb{R}^{2} .
$$

Applying the gradient estimate (3.15) in [25] and the above exponential decay of $\hat{v}_{a}(x)$, we finally obtain that

$$
\left|\nabla \tilde{v}_{a}(x)\right| \leq C \alpha_{a}^{4} e^{-\frac{|x|}{2}} \text { in } \mathbb{R}^{2}
$$

as $a \nearrow a^{*}$, which therefore completes the proof of Lemma A.2.

Acknowledgements: The authors are very grateful to the referees for many valuable suggestions which lead to the great improvements of the present paper. The authors also thank Prof. Robert Seiringer very much for his helpful discussions and interests on the subject of the present paper.

\section{References}

[1] J. R. Abo-Shaeer, C. Raman, J. M. Vogels and W. Ketterle, Observation of vortex lattices in Bose-Einstein condensate, Science 292 (2001), 476.

[2] A. Aftalion, Vortices in Bose-Einstein condensates, Progress in Nonlinear Differential Equations and their Applications, 67. Birkhäuser Boston, Inc., Boston, MA, 2006.

[3] A. Aftalion, S. Alama and L. Bronsard, Giant vortex and the breakdown of strong pinning in a rotating Bose-Einstein condensate, Arch. Rational Mech. Anal. 178 (2005), 247-286.

[4] A. Aftalion, R. L. Jerrard and J. Royo-Letelier, Non-existence of vortices in the small density region of a condensate, J. Funct. Anal. 260 (2011), 2387-2406.

[5] M. H. Anderson, J. R. Ensher, M. R. Matthews, C. E. Wieman and E. A. Cornell, Observation of Bose-Einstein condensation in a dilute atomic vapor, Science 269 (1995), 198-201.

[6] J. Arbunich, I. Nenciu and C. Sparber, Stability and instability properties of rotating Bose-Einstein condensates, Lett. Math. Phys. 109 (2019), 1415-1432.

[7] G. Arioli and A. Szulkin, A semilinear Schrödinger equation in the presence of a magnetic field, Arch. Ration. Mech. Anal. 170 (2003), 277-295.

[8] W. Z. Bao and Y. Y. Cai, Ground states of two-component Bose-Einstein condensates with an internal atomic Josephson junction, East Asia J. Appl. Math. 1 (2011), 49-81.

[9] I. Bloch, J. Dalibard and W. Zwerger, Many-body physics with ultracold gases, Reviews of Modern Phys. 80 (2008), 885-964.

[10] C. C. Bradley, C. A. Sackett and R. G. Hulet, Bose-Einstein condensation of lithium: observation of limited condensate number, Phys. Rev. Lett. 78 (1997), 985.

[11] C. C. Bradley, C. A. Sackett, J. J. Tollett and R. G. Hulet, Evidence of Bose-Einstein condensation in an atomic gas with attractive interactions, Phys. Rev. Lett. 75 (1995), 1687. Erratum Phys. Rev. Lett. 79 (1997), 1170.

[12] J. Byeon and Y. Oshita, Uniqueness of standing waves for nonlinear Schrödinger equations, Proc. Roy. Soc. Edinburgh Sect. A 138 (2008), 975-987. 
[13] J. Byeon and Z. Q. Wang, Standing waves with a critical frequency for nonlinear Schrödinger equations, Arch. Rational Mech. Anal. 165 (2002), 295-316.

[14] D. M. Cao and Z. W. Tang, Existence and uniqueness of multi-bump bound states of nonlinear Schrodinger equations with electromagnetic fields, J. Differential Equations 222 (2006), no. 2, 381-424.

[15] L. D. Carr and C. W. Clark, Vortices in attractive Bose-Einstein condensates in two dimensions, Phys. Rev. Lett. 97 (2006), 010403.

[16] T. Cazenave, Semilinear Schrödinger equations, Courant Lecture Notes in Mathematics Vol. 10, Courant Institute of Mathematical Science/AMS, New York, 2003.

[17] M. Correggi and D. Dimonte, On the third critical speed for rotating Bose-Einstein condensates, J. Math. Phys. 57 (2016), 071901.

[18] M. Correggi, F. Pinsker, N. Rougerie and J. Yngvason, Critical rotational speeds for superfluids in homogeneous traps, J. Math. Phys. 53 (2012), 095203.

[19] M. Correggi and N. Rougerie, Boundary behavior of the Ginzburg-Landau order parameter in the surface superconductivity regime, Arch. Rational Mech. Anal. 219 (2016), 553-606.

[20] M. Correggi, N. Rougerie and J. Yngvason, The transition to a giant vortex phase in a fast rotating Bose-Einstein condensate, Comm. Math. Phys. 303 (2011), 451-508.

[21] F. Dalfovo, S. Giorgini, L. P. Pitaevskii and S. Stringari, Theory of Bose-Einstein condensation in trapped gases, Reviews of Modern Phys. 71 (1999), 463-512.

[22] M. J. Esteban and P. L. Lions, Stationary solutions of nonlinear Schrödinger equations with an external magnetic field, Partial differential equations and the calculus of variations, Vol. I, 401-449, Progr. Nonlinear Differential Equations Appl. 1, Birkhuser Boston, Boston, MA, 1989.

[23] A. L. Fetter, Rotating trapped Bose-Einstein condensates, Reviews of Modern Phys. 81 (2009), 647-691.

[24] B. Gidas, W. M. Ni and L. Nirenberg, Symmetry of positive solutions of nonlinear elliptic equations in $\mathbb{R}^{n}$, Mathematical analysis and applications Part A, Adv. in Math. Suppl. Stud. Vol. 7, Academic Press, New York (1981), 369-402.

[25] D. Gilbarg and N. S. Trudinger, Elliptic Partial Differential Equations of Second Order, Springer, 1997.

[26] E. P. Gross, Structure of a quantized vortex in boson systems, Nuovo Cimento 20 (1961), $454-466$.

[27] E. P. Gross, Hydrodynamics of a superfluid condensate, J. Math. Phys. 4 (1963), 195-207.

[28] M. Grossi, On the number of single-peak solutions of the nonlinear Schrödinger equations, Ann. Inst. H. Poincaré Anal. Non Linéaire 19 (2002), 261-280.

[29] Y. J. Guo, C. S. Lin and J. C. Wei, Local uniqueness and refined spike profiles of ground states for two-dimensional attractive Bose-Einstein condensates, SIAM J. Math. Anal. 49 (2017), 3671-3715.

[30] Y. J. Guo and R. Seiringer, On the mass concentration for Bose-Einstein condensates with attractive interactions, Lett. Math. Phys. 104 (2014), 141-156.

[31] Y. J. Guo, Z. Q. Wang, X. Y. Zeng and H. S. Zhou, Properties of ground states of attractive Gross-Pitaevskii equations with multi-well potentials, Nonlinearity 31 (2018), 957-979. 
[32] Y. J. Guo, X. Y. Zeng and H. S. Zhou, Energy estimates and symmetry breaking in attractive Bose-Einstein condensates with ring-shaped potentials, Ann. Inst. H. Poincaré Anal. Non Linéaire 33 (2016), 809-828.

[33] Q. Han and F. H. Lin, Elliptic Partial Differential Equations, Courant Lecture Note in Math. 1, Courant Institute of Mathematical Science/AMS, New York, 2011.

[34] C. Huepe, S. Metens, G. Dewel, P. Borckmans and M.E. Brachet, Decay rates in attractive Bose-Einstein condensates, Phys. Rev. Lett. 82 (1999), 1616-1619.

[35] R. Ignat and V. Millot, The critical velocity for vortex existence in a two-dimensional rotating Bose-Einstein condensate, J. Funct. Anal. 233 (2006), 260-306.

[36] R. Ignat and V. Millot, Energy expansion and vortex location for a two-dimensional rotating Bose-Einstein condensate, Rev. Math. Phys. 18 (2006), 119-162.

[37] Y. Kagan, A.E. Muryshev and G.V. Shlyapnikov, Collapse and Bose-Einstein condensation in a trapped Bose gas with nagative scattering length, Phys. Rev. Lett. 81 (1998), 933-937.

[38] K. Kasamatsu, M. Tsubota and M. Ueda, Giant hole and circular superflow in a fast rotating Bose-Einstein condensate, Phys. Rev. B 66 (2002), 053606.

[39] K. Kurata, Existence and semi-classical limit of the least energy solution to a nonlinear Schrödinger equation with electromagnetic fields, Nonlinear Anal. 41 (2000), 763-778.

[40] M. K. Kwong, Uniqueness of positive solutions of $\Delta u-u+u^{p}=0$ in $\mathbb{R}^{N}$, Arch. Rational Mech. Anal. 105 (1989), 243-266.

[41] M. Lewin, P. T. Nam and N. Rougerie, Blow-up profile of rotating 2D focusing Bose gases, Macroscopic Limits of Quantum Systems, Springer Verlag, 2018.

[42] E. H. Lieb and M. Loss, Analysis, Graduate Studies in Mathematics Vol. 14. Amer. Math. Soc., Providence, RI, second edition, 2001.

[43] E. H. Lieb and R. Seiringer, Derivation of the Gross-Pitaevskii equation for rotating Bose gases, Comm. Math. Phys. 264 (2006), 505-537.

[44] E. H. Lieb, R. Seiringer, J. P. Solovej and J. Yngvason, The mathematics of the Bose gas and its condensation, Oberwolfach Seminars, 34 Birkhäuser Verlag, Basel, 2005.

[45] E. H. Lieb, R. Seiringer and J. Yngvason, Bosons in a trap: A rigorous derivation of the Gross-Pitaevskii energy functional, Phys. Rev. A 61 (2000), 043602.

[46] M. Loss and B. Thaller, Optimal heat kernel estimates for Schrödinger operators with magnetic field in two dimensions, Comm. Math. Phys. 186 (1997), 95-107.

[47] E. Lundh, A. Collin, and K.-A. Suominen, Rotational states of Bose gases with attractive interactions in anharmonic traps, Phys. Rev. Lett. 92 (2004), 070401.

[48] W.-M. Ni and I. Takagi, On the shape of least-energy solutions to a semilinear Neumann problem, Comm. Pure Appl. Math. 44 (1991), 819-851.

[49] W.-M. Ni and I. Takagi, Locating the peaks of least-energy solutions to a semilinear Neumann problem, Duke Math. J. 70 (1993), 247-281.

[50] N. Okazawa, An $L^{p}$ theory for Schrödinger operator with nonnegative potentials, J. Math. Soc. Japan. 36 (1984), 675-688.

[51] L. P. Pitaevskii, Vortex lines in an imperfect Bose gas, Sov. Phys. JETP. 13 (1961), $451-454$. 
[52] M. Reed and B. Simon, Methods of modern mathematical physics. IV. Analysis of operators, Academic Press, New York-London, 1978.

[53] N. Rougerie, The giant vortex state for a Bose-Einstein condensate in a rotating anharmonic trap: extreme rotation regimes, J. Math. Pures Appl. 9 (2011), 296-347.

[54] C. A. Sackett, H. T. C. Stoof and R. G. Hulet, Growth and collapse of a Bose-Einstein condensate with attractive interactions, Phys. Rev. Lett. 80 (1998), 2031.

[55] E. Sandier and S. Serfaty, Global minimizers for the Ginzburg-Landau functional below the first critical magnetic field, Ann. Inst. H. Poincaré Anal. Non Linéaire 17 (2000), $119-145$.

[56] E. Sandier and S. Serfaty, Vortices in the Magnetic Ginzburg-Landau Model, Progress in Nonlinear Differential Equations and their Applications 70, Basel: Birkháuser, 2007.

[57] S. Secchi and M. Squassina, On the location of spikes for the Schrödinger equation with electromagnetic field, Comm. Contemp. Math. 7 (2005), 251-268.

[58] R. Seiringer, Gross-Pitaevskii theory of the rotating Bose gas, Comm. Math. Phys. 229 (2002), 491-509.

[59] M. Wadati and T. Tsurumi, Critical number of atoms for the magnetically trapped BoseEinstein condensate with negative s-wave scattering length, Phys. Lett. A 247 (1998), 287-293.

[60] X. F. Wang, On concentration of positive bound states of nonlinear Schrödinger equations, Comm. Math. Phys. 153 (1993), 229-244.

[61] M. I. Weinstein, Nonlinear Schrödinger equations and sharp interpolations estimates, Comm. Math. Phys. 87 (1983), 567-576.

[62] N. K. Wilkin, J. M. F. Gunn and R. A. Smith, Do attractive Bosons condense?, Phys. Rev. Lett. 80 (1998), 2265.

[63] J. Zhang, Stability of standing waves for nonlinear Schrödinger equations with unbounded potentials, Z. Angew. Math. Phys. 51 (2000), 498-503.

[64] J. Zhang, Stability of attractive Bose-Einstein condensates, J. Stat. Phys. 101 (2000), $731-746$.

[65] M. W. Zwierlein, J. R. Abo-Shaeer, A. Schirotzek, C. H. Schunck and W. Ketterle, Vortices and superfluidity in a strongly interacting fermi gas, Nature 435 (2005), 10471051. 